\title{
Spectral and scattering theory for the Laplacian on asymptotically Euclidian spaces
}

\author{
Richard B. Melrose \\ Massachusetts Institute of Technology
}

\section{Introduction.}

There are many approaches to conventional Euclidian scattering theory. In this exposition an essentially microlocal view is adopted. Apart from its intrinsic interest this is intended as preparation for later generalization, to more complicated geometric settings. In fact, the treatment given here extends beyond the usual confines of scattering theory in that the spectral and scattering theory, at least the elementary part, is covered for the Laplacian associated to a 'scattering metric' on any compact manifold with boundary.

By a scattering metric on a compact manifold with boundary, $X$, we shall mean a Riemann metric on the interior of $X$ which can be brought to the form

$$
g_{\mathrm{sc}}=\frac{d x^{2}}{x^{4}}+\frac{h}{x^{2}}
$$

where $x \in \mathcal{C}^{\infty}(X)$ is a boundary defining function and $h$ is a smooth symmetric 2-tensor on $X$ which is non-degenerate when restricted to the boundary. Clearly the existence of $x$ and $h$ such that (1) holds is only a limitation on the behaviour of the metric near the boundary. Such a metric is asymptotically flat in the sense that the Riemann curvature tensor extends smoothly up to the boundary and vanishes there.

One virtue of scattering metrics is that they exist on any compact manifold with boundary and in this sense the theory is universal. The prototypical example is

Euclidian space. Namely if $\mathbb{R}^{n}$ is compactified to a unit hemisphere by stereographic projection, as discussed in $\S 1$, then the Euclidian metric takes the form (1) near the boundary.

A scattering metric is a, rather special, example of a metric on the structure bundle of the Lie algebra $\mathcal{V}_{\mathrm{sc}}(X)=x \mathcal{V}_{\mathrm{b}}(X)$, where $\mathcal{V}_{\mathrm{b}}(X)$ is the Lie algebra of all smooth vector fields on $X$ which are tangent to the boundary. The space $\mathcal{V}_{\mathrm{sc}}(X)$ is a boundary-fibration structure (which notion we do not define or use here) in the sense of [8], with structure bundle

$$
{ }^{\mathrm{sc}} T X=1 / x \cdot{ }^{\mathrm{b}} T X
$$

this is discussed in $\S 2$. The basic approach taken here and examined in some generality in [8] is that the boundary-fibration structure, in this case just $\mathcal{V}_{\mathrm{sc}}(X)$, 
should be thought of as a geometry. This approach is based on the development of a calculus of pseudodifferential operators, appropriate to the context and coming from the geometry. This calculus can then be applied, much as the usual calculus of pseudodifferential operators on a compact manifold without boundary can be applied, to give regularity, and in particular propagation, results.

Here the 'small calculus' of scattering pseudodifferential operators is discussed and used to prove commutator estimates analogous to those found in scattering theory on Euclidian space. In particular we give a variant of Mourre's estimate ([11]) in this setting. For the half-sphere, $\mathbb{S}_{+}^{n}$, this calculus reduces to one of the standard versions of the global pseudodifferential calculus on $\mathbb{R}^{n}$, via stereographic projection.

In fact three examples of boundary-fibration structures occur here; they can be recognized by the elliptic operators associated to each. Let $x \geq 0$ be a coordinate defining the boundary (which is 'at infinity') and let $y=\left(y_{1}, \ldots, y_{n-1}\right)$ be tangential coordinates. Then, with $D_{x}=-i \partial / \partial x$ and $D_{y}=-i \partial / \partial y$, typical (invertible) elliptic operators are

$$
\begin{array}{rl}
\left(x D_{x}\right)^{2}+\left|D_{y}\right|^{2}+1 & \mathrm{~b}(=\text { boundary }) \text {-calculus } \\
\left(x^{2} D_{x}\right)^{2}+\left|D_{y}\right|^{2}+1 & \mathrm{c}(=\text { cusp }) \text {-calculus } \\
\left(x^{2} D_{x}\right)^{2}+\left|x D_{y}\right|^{2}+1 & \mathrm{sc}(=\text { scattering }) \text {-calculus. }
\end{array}
$$

Although the cusp calculus is not discussed in detail here, it would be natural to do so since its use makes the regularity results of $\S 11$ more precise. Whereas the scattering calculus is tempered, in the sense that it is locally contained in the Weyl calculus of Hörmander [5], this is not the case for either the boundary or cusp calculi.

The material below falls naturally into three parts, the contents of which are described in more detail below. In $\S \S 1-7$ the quantization of the Lie algebra of vector fields associated to the metric (1) is explained. The central sections, $\S \S 8$ 16 contain the main analytic results, concerning the behaviour of the limit of the resolvent of the Laplacian on the spectrum and leading in particular to the definition of the scattering operator. In the third part, consisting of the appendices, the direct and intrinsic approach to the quantization is outlined.

The properties of the scattering operator in this generality remain to be investigated. In the Euclidian context, the scattering operator (at fixed energy) as defined in $\S 15$ is of the form $R+A$, where $R$ is the anitpodal reflection of the sphere and $A$ is a smoothing operator. In the general case of a scattering metric the appropriate generalization is expected to be:

CONJECTURE. The scattering operator associated to a scattering metric is a Fourier integral operator with canonical relation the geodesic flow, at time $\pi$, of the boundary metric. 
This can be construed as the statement that, for a metric (1), infinity always has width $\pi$.

Other topics which remain to be treated include:

a) The behaviour of the resolvent near $\lambda=0$. This is considerably more complicated than in the Euclidian setting since it is influenced strongly by the eigenvalues of the induced Laplacian on $\partial X$.

b) The asymptotic behaviour of the resolvent kernel.

c) The derivation of a trace formula.

The central result proved below, as Proposition 12, is that the $\lambda$-outgoing solution to $\left(\Delta-\lambda^{2}\right) u=f$, where $f \in \mathcal{C}^{\infty}(X)$ vanishes with all its derivatives at $\partial X$ and $0 \neq \lambda \in \mathbb{R}$, has a complete asymptotic expansion with leading term

$$
u \sim e^{i \lambda / x} a(\theta) x^{\frac{1}{2}(n-1)}+O\left(x^{\frac{1}{2}(n+1)}\right), a \in \mathcal{C}^{\infty}(\partial X) .
$$

This result, well known in the Euclidian setting, can be viewed as either a strong form of the Sommerfeld radiation condition or a weak form of b) above. The space of square-integrable functions with respect to the metric volume form is written here $L_{\mathrm{sc}}^{2}(X)$. Then $u \in x^{t} L_{\mathrm{sc}}^{2}(X)$ for $t<-\frac{1}{2}$. Moreover if $u \in x^{t} L_{\mathrm{sc}}^{2}(X)$ for any $t \geq-\frac{1}{2}$ then $a \equiv 0$ and $u$ vanishes rapidly at $\partial X$. The critical exponent $t=-\frac{1}{2}$ is seen to arise from the commutator formula in the calculus of scattering pseudodifferential operators.

The structure of scattering differential operators is discussed in $\S 2$, after it is shown in $\S 1$ that stereographic projection, i.e. radial compactification, of $\mathbb{R}^{n}$ converts the translation invariance to such a structure. The properties of the Laplacian, acting on forms, associated to a metric (1), are examined in $\S 3$. The calculus of pseudodifferential operators obtained by quantization of the structure algebra is introduced in $\S 4$ by reduction to $\mathbb{R}^{n}$ and many of its properties are described in $\S 5$; although full proof are not given. Further discussion is relegated to Appendix B. In $\S 6$ the properties of the calculus are used to show that the resolvent family for the Laplacian of $(1)$ is holomorphic in $\mathbb{C} \backslash[0, \infty)$ with values in the calculus. The wavefront set associated to the scattering calculus, and a uniform version of it, is investigated in $\S 7$; this language is used as a convenient way to state microlocal estimates.

The first such estimates, reducing to Hörmander's propagation theorem for operators of real-principal type, are given in $\S 8$. The more subtle estimates, again proved by the positive-commutator method, around the radial surfaces for the operator $\Delta-\lambda^{2}$, are given in $\S 9$. These are the main estimates used in the subsequent analysis and amount to a version in this context of Mourre's estimate for the $\mathrm{N}$ body problem. In $\S 10$ these basic estimates, together with a unique continuation theorem, or Aronszajn-Cordes type, proved by Hörmander ([6]), show the absence of $L^{2}$ eigenfunctions for the Laplacian. Various forms of the Sommerfeld radiation condition are discussed in $\S 11$ and the asymptotic expansion of $\lambda$-outgoing solutions 
is deduced in $\S 12$. The boundary pairing for asymptotic data in $\S 13$ is used in $\S 14$ to prove the limiting absorption principle, i.e. to examine the limit of the resolvent as the parameter approaches the spectrum. Finally in $\S 15$ these results are used to define the scattering operator for the Laplacian. In $\S 16$ the minor modifications needed to treat long-range potentials are described.

The discussion below is intended to provide a bridge between the traditional methods of Euclidian scattering theory, which depends ultimately on the explicit inversion of the Euclidian Laplacian, and the more geometric problems encountered in the analysis of the Laplacian on complete Riemannian manifolds with uniform degeneracy at infinity, as typified by a metric singular at the boundary of a compact manifold with boundary. Indeed these scattering metrics form just such a class, but there are others of interest. Although all the results proved here are well known in the Euclidian setting we give proofs in this wider context. It is anticipated that the methods used here can be further generalized to give a unified treatment of the $N$-body problem and classes of singular metrics on manifolds with corners. For a recent discussion of the N-body problem see [13].

\section{Section 1: Stereographic projection.}

Consider the map

$$
\begin{gathered}
\mathrm{SP}: \mathbb{R}^{n} \ni z \longmapsto\left(\frac{1}{\left(1+|z|^{2}\right)^{\frac{1}{2}}}, \frac{z}{\left(1+|z|^{2}\right)^{\frac{1}{2}}}\right) \in \mathbb{S}_{+}^{n} \\
\mathbb{S}_{+}^{n}=\left\{t=\left(t_{0}, \ldots, t_{n}\right) \in \mathbb{R}^{n+1} ; t_{0} \geq 0,|t|=1\right\} .
\end{gathered}
$$

This is clearly an isomorphism onto the interior of $\mathbb{S}_{+}^{n}$, so it compactifies $\mathbb{R}^{n}$. We call this the radial compactification. Indeed the function $t_{0}=\left(1+|z|^{2}\right)^{-\frac{1}{2}}$ is a defining function for the boundary of $\mathbb{S}_{+}^{n}$, hence so is $x=1 /|z|$, which is defined in $t_{0}<1$. If we identify the collar neighbourhood $\left\{0 \leq t_{0}<1\right\} \subset \mathbb{S}_{+}^{n}$ of the boundary of $\mathbb{S}_{+}^{n}$ with $[0, \infty) \times \mathbb{S}^{n-1}$ by

$$
t \longmapsto\left(\frac{t_{0}}{\sqrt{1-t_{0}^{2}}}, \frac{t^{\prime}}{\sqrt{1-t_{0}^{2}}}\right)=(x, \omega), t^{\prime}=\left(t_{1}, \ldots, t_{n}\right)
$$

the Euclidian metric becomes

$$
|d z|^{2}=d r^{2}+r^{2} d \omega^{2}=\frac{d x^{2}}{x^{4}}+\frac{|d \omega|^{2}}{x^{2}}, r=\frac{1}{x}
$$

where $|d \omega|^{2}$ is the standard metric on the sphere. Thus under the radial compactification of $\mathbb{R}^{n}$ the Euclidian metric is transformed to a scattering metric, as in (1), on $\mathbb{S}_{+}^{n}$. 
Lemma 1. The group of affine-linear transformations of $\mathbb{R}^{n}$ lifts under SP to a group of global diffeomorphisms of $\mathbb{S}_{+}^{n}$; the linear vector fields lift to span, over $\mathcal{C}^{\infty}\left(\mathbb{S}_{+}^{n}\right)$, the space $\mathcal{V}_{\mathrm{b}}\left(\mathbb{S}_{+}^{n}\right)$ of all smooth vector fields tangent to the boundary and the constant vector fields on $\mathbb{R}^{n}$ lift to span, over $\mathcal{C}^{\infty}\left(\mathbb{S}_{+}^{n}\right), \mathcal{V}_{\mathrm{sc}}\left(\mathbb{S}_{+}^{n}\right)=t_{0} \mathcal{V}_{\mathrm{b}}\left(\mathbb{S}_{+}^{n}\right)$.

Proof: The group of affine-linear transformations is the semi-direct product of $\operatorname{Gl}(n, \mathbb{R})$ and the translation group, so it suffices to show that both of these lift to groups of diffeomorphisms. Exponentiation of linear vector fields gives a neighbourhood of the identity in $\operatorname{Gl}(n, \mathbb{R})$ and this, together with the coordinate reflections, generates the whole group; the reflections obviously lift to global diffeomorphisms. Similarly translations are just exponentials of constant vector fields so it suffices to prove the second part of the lemma.

Consider the basis $z_{i} \partial / \partial z_{j}$ of the linear vector fields. These are invariant under radial scaling, so when written in polar coordinates $x=r \omega, \omega \in \mathbb{S}^{n-1}$ they take the form

$$
z_{i} \frac{\partial}{\partial z_{j}}=a_{i j}(\omega) r \frac{\partial}{\partial r}+V_{i j}
$$

where $a_{i j} \in \mathcal{C}^{\infty}\left(\mathbb{S}^{n-1}\right)$ and $V_{i j} \in \mathcal{V}\left(\mathbb{S}^{n-1}\right)$ is a smooth vector field on the sphere. Thus under the radial inversion, $x=1 /|z|$, we find

$$
\mathrm{SP}_{*}\left(z_{i} \frac{\partial}{\partial z_{j}}\right)=-a_{i j} x \frac{\partial}{\partial x}+V_{i j}
$$

is a smooth vector field on $\mathbb{S}_{+}^{n}$ and is tangent to the boundary. Furthermore these span all the vector fields tangent to the boundary, a basis for which is given by $-x \partial / \partial x$, which is the lift of $z \cdot \partial / \partial z$, and the smooth vector fields on the sphere.

Similarly the constant vector fields, written in polar coordinates, take the form

$$
\frac{\partial}{\partial x_{j}}=b_{j}(\omega) \frac{\partial}{\partial r}+\frac{1}{r} W_{j}
$$

where $W_{j}$ is a smooth vector field on the sphere. Under the radial inversion this gives

$$
\mathrm{SP}_{*}\left(\frac{\partial}{\partial x_{j}}\right)=x\left(-b_{j}(\omega) x \frac{\partial}{\partial x}+W_{j}\right)
$$

Clearly these are elements of $\mathcal{V}_{\mathrm{sc}}(X)=x \mathcal{V}_{\mathrm{b}}\left(\mathbb{S}_{+}^{n}\right)$ and span this space over $\mathcal{C}^{\infty}\left(\mathbb{S}_{+}^{n}\right)$.

The fact that the linear transformations lift to be smooth on the radial compactification shows that there is a natural radial compactification of any vector space. Thus if $V$ is a vector space then

$$
\bar{V}=V \sqcup\left((V \backslash\{0\}) / \mathbb{R}^{+}\right) \approx \mathbb{S}_{+}^{n}
$$


has a natural smooth structure, independent of the basis used to define it. Furthermore Lemma 1 shows that this also varies smoothly with parameters. In particular if $V \longrightarrow M$ is a vector bundle over a manifold $M$ then the radial compactification $\bar{V}$ is a ball (really a half-sphere) bundle over $M$ with fibre $\bar{V}_{m}$ at $m \in M$, where $V_{m}$ is the fibre of $V$.

\section{Section 2: Structure algebra.}

Let $X$ be a general compact manifold with boundary and let $x \in \mathcal{C}^{\infty}(X)$ be a defining function for the boundary. Consider the space $\mathcal{V}_{\mathrm{sc}}(X)=x \mathcal{V}_{\mathrm{b}}(X)$ of smooth vector fields which are the product of $x$ and a smooth vector field on $X$ which is tangent to the boundary. That is, $V \in \mathcal{V}_{\mathrm{b}}(X)$ if and only if $V x \in x \mathcal{C}^{\infty}(X)$. If $x$ is any defining function for the boundary of $X$ then a neighbourhood of the boundary in $X$ is diffeomorphic to the product

$$
\partial X \supset O \equiv[0, \epsilon)_{x} \times \partial X, \epsilon>0 .
$$

In terms of any such decomposition $\mathcal{V}_{\mathrm{sc}}(X)$ is spanned, over $\Omega$, by $x^{2} D_{x}$ and $x \mathcal{V}(\partial X)$ where $\mathcal{V}(\partial X)$ is the space of all smooth vector fields on $\partial X$.

We shall denote by Diff* ${ }_{\mathrm{sc}}^{*}(X)$ the 'enveloping algebra' of $\mathcal{V}_{\mathrm{sc}}(X)$, meaning the ring of operators on $\mathcal{C}^{\infty}(X)$ generated by $\mathcal{V}_{\mathrm{sc}}(X)$ and $\mathcal{C}^{\infty}(X)$. As is shown below in this case the Laplacian of a scattering metric, acting on functions, is an element of $\operatorname{Diff}_{\mathrm{sc}}^{2}(X)$, i.e. can be written everywhere locally in the form

$$
\Delta=\sum_{j, k} a^{j k} V_{j} V_{k}+\sum_{j} b^{j} V_{j}
$$

where the $V_{j}$ form a local basis of $\mathcal{V}_{\mathrm{sc}}(X)$ and the coefficients are smooth. In fact $a^{j k}$ is the dual metric expressed with respect to this basis, so is positive definite. To examine the structure of this operator in detail, especially near the boundary, we shall consider two maps from $\operatorname{Diff}_{\mathrm{sc}}^{*}(X)$. The first is the symbol map, the second is the normal homomorphism. We subsequently combine them into a 'joint symbol.'

The symbol map is simply the principal part of the operator, its existence depends on the Lie algebra structure. On $\mathcal{V}_{\mathrm{sc}}(X)$

$$
\sigma_{\mathrm{sc}, 1}: \mathcal{V}_{\mathrm{sc}}(X) \longrightarrow \mathcal{C}^{\infty}\left(X ;{ }^{\mathrm{sc}} T^{*} X\right), \sigma_{\mathrm{sc}, 1}(V)=i V
$$

is defined by the identification of a vector field as a section of ${ }^{\mathrm{sc}} T X$, with a linear map on the fibres of the dual bundle. This extends multiplicatively to define the symbol on differential operators of order $m$ :

$$
\sigma_{\mathrm{sc}, m}: \operatorname{Diff}_{\mathrm{sc}}^{m}(X) \longrightarrow P^{[m]}\left({ }^{\mathrm{sc}} T^{*} X\right)
$$

with the symbol a homogeneous polynomial of degree $m$ on the fibres of ${ }^{\mathrm{sc}} T^{*} X$. The symbol map gives a short exact sequence:

$$
0 \longrightarrow \operatorname{Diff}_{\mathrm{sc}}^{m-1}(X) \longrightarrow \operatorname{Diff}_{\mathrm{sc}}^{m}(X) \stackrel{\sigma_{\mathrm{sc}, m}}{\longrightarrow} P^{[m]}\left({ }^{\mathrm{sc}} T^{*} X\right) \longrightarrow 0 .
$$


The second map arises by freezing the coefficients at a boundary point. Since $\mathcal{V}_{\mathrm{b}}(X)$ is a Lie algebra with $\mathcal{V}_{\mathrm{b}}(X) \cdot x \subset x \mathcal{C}^{\infty}(X)$,

$$
\left[\mathcal{V}_{\mathrm{sc}}(X), \mathcal{V}_{\mathrm{sc}}(X)\right]=\left[x \mathcal{V}_{\mathrm{b}}(X), x \mathcal{V}_{\mathrm{b}}(X)\right] \subset x^{2} \mathcal{V}_{\mathrm{b}}(X)=x \mathcal{V}_{\mathrm{sc}}(X)
$$

Thus the evaluation map

$$
N_{\mathrm{sc}, p}: \mathcal{V}_{\mathrm{sc}}(X) \longrightarrow{ }^{\mathrm{sc}} T_{p} X, p \in \partial X
$$

is a Lie algebra homomorphism into a commutative algebra. With the corresponding restriction operation on functions, $\mathcal{C}^{\infty}(X) \ni f \longmapsto f(p) \in \mathbb{C}$, for $p \in \partial X$, thus extends to define the normal homomorphism at $p$ :

$$
N_{\mathrm{sc}, p}: \operatorname{Diff}_{\mathrm{sc}}^{m}(X) \longrightarrow \operatorname{Diff}_{\mathrm{I}}^{m}\left({ }^{\mathrm{sc}} T_{p} X\right)
$$

where the range space consists of the translation-invariant (i.e. constant coefficient) differential operators on the vector space ${ }^{\mathrm{sc}} T_{p} X$. For a vector bundle, $U$, we shall denote by $\operatorname{Diff}_{\mathrm{I}}^{m}(U)$ the space of such translation-invariant differential operators on the fibres varying smoothly with the base point. Again this leads to a short exact sequence

$$
0 \longrightarrow x \operatorname{Diff}_{\mathrm{sc}}^{m}(X) \longrightarrow \operatorname{Diff}_{\mathrm{sc}}^{m}(X) \longrightarrow \operatorname{Diff}_{\mathrm{I}}^{m}\left({ }^{\mathrm{sc}} T X\right) \longrightarrow 0 .
$$

A constant coefficient differential operator acting on functions on a vector space is reduced to multiplication by a polynomial by the invariant Fourier transform (to smooth densities on the dual space). Thus the normal operator can also be reinterpreted as a map to polynomials:

$$
\widehat{N}_{\mathrm{sc}, p}: \operatorname{Diff}_{\mathrm{sc}}^{m}(X) \longrightarrow P^{m}\left({ }^{\mathrm{sc}} T_{p}^{*} X\right), p \in \partial X
$$

The range here is the space of all, not just the homogeneous, polynomials of degree $m$. For $P \in \operatorname{Diff}_{\mathrm{sc}}^{m}(X), \widehat{N}_{\mathrm{sc}, p}(P)$ will be called the normal symbol of $P$ at $p$.

It is important to understand the precise relationship between the symbols $\sigma_{\mathrm{sc}, m}(P)$ and $\widehat{N}_{\text {sc }, p}(P)$. In fact the only relationship in general is the obvious one that the leading part of the normal symbol is the restriction of the symbol to the boundary:

$$
\widehat{N}_{\mathrm{sc}, p}(P)-\sigma_{\mathrm{sc}, m}(P) \in P^{m-1}\left({ }^{\mathrm{sc}} T_{p}^{*} X\right), p \in \partial X .
$$

We shall write $j_{\mathrm{sc}, m}(P)=\left(\sigma_{\mathrm{sc}, m}(P), \widehat{N}_{\mathrm{sc}}(P)\right)$ for the joint symbol which takes values is

${ }^{\mathrm{sc}} \widetilde{P}^{m, 0}(X)=\left\{(p, \widehat{N}) \in P^{[m]}\left({ }^{\mathrm{sc}} T^{*} X\right) \times P^{m}\left({ }^{\mathrm{sc}} T_{\partial X}^{*} X\right) ; \widehat{N}-p_{\lceil\partial X} \in P^{m-1}\left({ }^{\mathrm{sc}} T_{\partial X}^{*} X\right)\right\}$.

Lemma 2. For any $P \in \operatorname{Diff}_{\mathrm{sc}}^{m}(X)$ the symbol and normal symbol are related by (2.11) and

$$
0 \longrightarrow x \operatorname{Diff}_{\mathrm{sc}}^{m-1}(X) \longrightarrow \operatorname{Diff}_{\mathrm{sc}}^{m}(X) \stackrel{j_{\mathrm{sc}, m}}{\longrightarrow}{ }^{\mathrm{sc}} \widetilde{P}^{m, 0}(X) \longrightarrow 0
$$

is a short exact sequence. 


\section{Section 3: Scattering Laplacian.}

For the Laplacian on functions we shall see below that

$$
\sigma_{\mathrm{sc}, 2}(\Delta)=|\zeta|^{2} \text { at } \zeta \in{ }^{\mathrm{sc}} T_{p}^{*} X, p \in X
$$

is the metric function and

$$
N_{\mathrm{sc}, p}(\Delta)=\Delta_{p}, p \in \partial X
$$

is just the flat Laplacian on ${ }^{\mathrm{sc}} T_{p} X$. Thus, for the Laplacian, (2.11) holds in the strong sense that $\widehat{N}_{\mathrm{sc}, p}(\Delta)=\sigma_{\mathrm{sc}, 2}(\Delta) \curlyvee_{\partial X}$, which leads to unfortunate confusion!

In fact the special form of the metric (1), as compared to a general fibre metric on ${ }^{\mathrm{sc}} T X$, allows $(3.2)$ to be refined considerably. For the enveloping algebra $\operatorname{Diff}_{\mathrm{b}}^{*}(X)$ of the Lie algebra $\mathcal{V}_{\mathrm{b}}(X)$ there are maps, discussed in [7], similar to those just described:

$$
\begin{aligned}
\sigma_{\mathrm{b}, m} & : \operatorname{Diff}_{\mathrm{b}}^{m}(X) \longrightarrow P^{[m]}\left({ }^{\mathrm{b}} T^{*} X\right) \\
I: \operatorname{Diff}_{\mathrm{b}}^{m}(X) & \left.\longrightarrow \operatorname{Diff}_{\mathrm{b}, \mathrm{I}}^{m} \overline{N^{+}} X\right) .
\end{aligned}
$$

Here $\overline{N^{+}} X$ is the radially compactified inward-pointing normal bundle to the boundary of $X$; this is a closed interval bundle over $\partial X$ and Diff ${ }_{\mathrm{b}, \mathrm{I}}^{m}\left(\overline{N^{+}} X\right)$ is the subspace of $\operatorname{Diff}_{\mathrm{b}}^{m}\left(\overline{N^{+}} X\right)$ consisting of the operators invariant under the natural $\mathbb{R}^{+}$-action on $\overline{N^{+}} X$.

Lemma 3. For the Laplacian of a metric (1), acting on functions,

$$
\Delta=x Q x, Q \in \operatorname{Diff}_{\mathrm{b}}^{2}(X), I(Q)=\left(x D_{x}\right)^{2}+i n x D_{x}-(n-1)+\Delta_{0}
$$

where $\Delta_{0}$ is the Laplacian on $\partial X$ of the metric $h\lceil\partial X$ and $d x$ is used to trivialize the normal bundle to $\partial X$.

Proof: Choose local coordinates near any point of the boundary, with $x$ as the normal variable and $y$ as tangential variables. To emphasize the similarity to the Euclidian case near, but not at, the boundary consider the local coordinates $r=1 / x$ and $y$ in terms of which the metric is of the form $d r^{2}+r^{2} h\left(x, y, d y,-r^{-2} d r\right)$. In any coordinates the Laplacian can be written

$$
\Delta=\sum_{j, k=0}^{n} \frac{1}{\sqrt{g}} D_{j} g^{i k} \sqrt{g} D_{k}
$$

where $g$ is the determinant of the metric $g_{j k}$. Applying this with $r=z_{0}, y_{j}=z_{j}$, the metric components satisfy

$$
\begin{aligned}
& g_{00}=1+O\left(r^{-2}\right), g_{0 j}=O(1), g_{i j}=r^{2}\left(\bar{h}_{i j}+O\left(r^{-1}\right)\right) \\
& g^{00}=1+O\left(r^{-2}\right), g^{0 j}=O\left(r^{-2}\right) \text { and } g^{i j}=r^{-2}\left(\bar{h}^{i j}+O\left(r^{-1}\right)\right)
\end{aligned}
$$


where $\bar{h}$ is the induced metric on the boundary.

It follows that the cross terms, with $j=0$ and $k \neq 0$ or $j \neq 0$ and $k=0$ vanish as $x^{3}$ when expressed in terms of $x \partial_{x}$ and $\partial_{y}$. Thus the computation is reduced to the case where $h$ does not depend on $d x$. Exactly as for the Laplacian on $\mathbb{R}^{n}$ expressed in terms of polar coordinates it follows that

$$
\Delta=D_{r}^{2}-i \frac{n-1}{r} D_{r}+r^{-2} \Delta_{0}+x^{3} \operatorname{Diff}_{\mathrm{b}}^{2}(X) .
$$

Reverting to the coordinate $x=1 / r$

$$
\Delta=\left(x^{2} D_{x}\right)^{2}+i(n-1) x^{3} D_{x}+x^{2} \Delta_{0}+x^{3} \operatorname{Diff}_{\mathrm{b}}^{2}(X)
$$

and commuting one factor of $x$ to the right gives (3.4).

For any $m$

$$
x^{m} \operatorname{Diff}_{\mathrm{b}}^{m}(X) \subset \operatorname{Diff}_{\mathrm{sc}}^{m}(X)
$$

and the normal operator of $x^{m} Q$, for $Q \in \operatorname{Diff}_{\mathrm{b}}^{m}(X)$, is easily computed from either the indicial operator of $Q$ or the symbol of $Q$ at the boundary:

$$
\widehat{N}_{\mathrm{sc}}\left(x^{m} Q\right)=x^{m} \sigma_{b, m}(I(Q)),
$$

where $\sigma_{b, m}(I(Q))$ is interpreted as a homogeneous polynomial of degree $m$ on the fibres of ${ }^{\mathrm{b}} T_{\partial X}^{*} X$ which becomes a homogeneous polynomial of degree $m$ on ${ }^{\mathrm{sc}} T_{\partial X}^{*} X$ after multiplication by $x^{m}$. In particular the normal operator of $x^{m} Q$ is always homogeneous of degree $m$, which is not the case for a general element of $\operatorname{Diff}_{\mathrm{sc}}^{m}(X)$.

It is convenient below to have a special type of extension of the boundary Laplacian to an operator on $X$. Such a possibility arises because the metric (1) forces a choice of boundary defining function, $x$, up to $O\left(x^{2}\right)$.

Let $\mathcal{V}_{\mathrm{c}}(X) \subset \mathcal{V}_{\mathrm{b}}(X)$ denote the Lie algebra of vector fields with the property

$$
V \in \mathcal{V}_{\mathrm{c}}(X) \Longleftrightarrow V \in \mathcal{V}_{\mathrm{b}}(X) \text { and } V x=0\left(x^{2}\right) .
$$

In terms of a product decomposition $(2.1), \mathcal{V}_{\mathrm{c}}(X)$ is spanned by $x^{2} D_{x}$ and $\mathcal{V}(\partial X)$; it depends on the choice of $x$. Any $W \in \mathcal{V}(\partial X)$ can be extended to $\widetilde{W} \in \mathcal{V}_{\mathrm{c}}(X)$. Let $\operatorname{Diff}_{\mathrm{c}}^{*}(X)$ be the enveloping algebra of $\mathcal{V}_{\mathrm{c}}(X)$. Using (2.1) to extend the boundary Laplacian to act on the second factor, and cutting off away from the boundary gives an extension

$$
\widetilde{\Delta}_{0} \in \operatorname{Diff}_{\mathrm{c}}^{2}(X), \widetilde{\Delta}_{0} u \uparrow=\Delta_{0}\left(u \Upsilon_{\partial X}\right) .
$$


The fact that $g^{00}=1+O\left(x^{2}\right)$ in (3.6) shows that, in terms of (2.1), $Q=$ $I(Q)+x Q^{\prime}$ where $I\left(Q^{\prime}\right)$ has no term $a\left(x D_{x}\right)^{2}$. Thus $x Q^{\prime} \in \operatorname{Diff}_{\mathrm{c}}^{2}(X)$, so together with (3.4) this implies

$$
\Delta=x I(Q) x+G \text { with } G \in x \operatorname{Diff}_{\mathrm{sc}}^{2}(X) \cap x^{2} \operatorname{Diff}_{\mathrm{c}}^{2}(X) .
$$

Notice that (3.4) shows that $G \in x^{3} \operatorname{Diff}_{\mathrm{b}}^{3}(X)$.

We interpret (3.12) as meaning that the metric (1) is 'short range.' To see the relationship of (3.12) to more traditional notions of short-range, consider the operator, $\Delta+V$, obtained by the addition of a potential $V \in \mathcal{C}^{\infty}(X)$. If $V_{\uparrow \partial X} \neq 0$ then the addition of $V$ completely disrupts (3.4), since it even changes the normal symbol. If $V_{\uparrow \partial X} \equiv 0, V=x V^{\prime}$, then the addition of $V$ still changes (3.4) substantially but the first part of (3.12) still holds:

$$
\Delta+V=x I(Q) x+x G^{\prime}, G^{\prime} \in \operatorname{Diff}_{\mathrm{sc}}^{2}(X), V \text { long-range. }
$$

If on the other hand $V=x^{2} V^{\prime \prime}$ then (3.4) is preserved in the sense that

$$
\Delta+V=x\left(Q+V^{\prime \prime}\right) x=x I(Q) x+G, G \text { as in }(3.12)
$$

in this case it is reasonable to say that such a potential is short-range. The general case could be described as 'very-long-range'.

For the proof of global regularity below it is important to examine the commutator of $\widetilde{\Delta}_{0}$ and $\Delta$.

Lemma 4. If $\widetilde{\Delta}_{0} \in \operatorname{Diff}_{\mathrm{c}}^{2}(X)$ is an extension of the boundary Laplacian into the enveloping algebra of $\mathcal{V}_{\mathrm{c}}(X)$ then

$$
\left[\Delta, \widetilde{\Delta}_{0}\right] \in x^{3} \operatorname{Diff}_{\mathrm{b}}^{3}(X) \cap x^{2} \operatorname{Diff}_{\mathrm{c}}^{3}(X) \subset \operatorname{Diff}_{\mathrm{sc}}^{3}(X) .
$$

If $V \in x \mathcal{C}^{\infty}(X)$ is long-range then

$$
\left[\Delta+V, \widetilde{\Delta}_{0}\right] \in\left(x^{2} \operatorname{Diff}_{\mathrm{c}}^{3}(X)+x \operatorname{Diff}_{\mathrm{c}}^{1}(X)\right) \subset \operatorname{Diff}_{\mathrm{sc}}^{3}(X) .
$$

If $V \in x^{2} \mathcal{C}^{\infty}(X)$ is short-range then

$$
\left[\Delta+V, \widetilde{\Delta}_{0}\right] \in x^{2} \operatorname{Diff}_{\mathrm{c}}^{3}(X) \subset \operatorname{Diff}_{\mathrm{sc}}^{3}(X) .
$$

Proof: Since $\widetilde{\Delta}_{0} \in \operatorname{Diff}_{\mathrm{c}}^{2}(X)$, the commutator $\left[\widetilde{\Delta}_{0}, x\right] \in x^{2} \operatorname{Diff}_{\mathrm{c}}^{1}(X) \subset x^{2} \operatorname{Diff}_{\mathrm{b}}^{1}(X)$. From Lemma 3 the commutator can be written

$$
\left[\Delta, \widetilde{\Delta}_{0}\right]=x Q\left[x, \widetilde{\Delta}_{0}\right]+x\left[Q, \widetilde{\Delta}_{0}\right] x+\left[x, \widetilde{\Delta}_{0}\right] Q x
$$


the first and the last term of which are therefore in $x^{3} \operatorname{Diff}_{\mathrm{b}}^{3}(X)$. The fact that $\widetilde{\Delta}_{0}$ restricts to the boundary to be $\Delta_{0}$ and $Q$ is given by (3.4) shows that the middle term in (3.18) is also in $x^{3} \operatorname{Diff}_{\mathrm{b}}^{3}(X)$. Thus $\left[\Delta, \widetilde{\Delta}_{0}\right] \subset x^{3} \operatorname{Diff}_{\mathrm{b}}^{3}(X)$.

The remainder of (3.15) follows from (3.12). Similarly (3.16) and (3.17) follow from the trivial observation that $\left[\widetilde{\Delta}_{0}, \mathcal{C}^{\infty}(X)\right] \subset \operatorname{Diff}_{\mathrm{c}}^{1}(X)$.

Although we shall mainly consider the Laplacian acting on functions it is hardly any more trouble to treat the case of the Laplacian on forms, which is again short range. Following the principle that ${ }^{\mathrm{sc}} T X$ represents the geometry of the problem, we shall replace the usual form bundles, $\Lambda^{k}(X)$, by the corresponding 'scattering' form bundles

$$
{ }^{\mathrm{sc}} \Lambda^{k} X=x^{-k} \cdot{ }^{\mathrm{b}} \Lambda^{k} X
$$

Thus $u \in \mathcal{C}^{\infty}\left(X ;{ }^{\mathrm{sc}} \Lambda^{k} X\right)$ means that $u$ is a smooth section of $\Lambda^{k} X$ over the interior of $X$ and that in any coordinates near the boundary, $x, y_{1}, y_{n-1}$ where $x$ locally defines the boundary,

$$
u=\sum_{|\alpha|=k-1} a_{\alpha}(x, y) \frac{d x}{x^{2}} \wedge \frac{d y^{\alpha}}{x^{k-1}}+\sum_{|\beta|=k} b_{\beta}(x, y) \frac{d y^{\beta}}{x^{k}}
$$

with the coefficients smooth up to $x=0$.

The space of operators $\operatorname{Diff}_{\mathrm{sc}}^{m}(X)$ is a left $\mathcal{C}^{\infty}(X)$-module, so the corresponding operators $\operatorname{Diff}_{\mathrm{sc}}^{m}(X ; E, F)$ from sections of one bundle, $E$, to sections of another bundle, $F$, can be defined as

$$
\operatorname{Diff}_{\mathrm{sc}}^{m}(X ; E, F)=\mathcal{C}^{\infty}(X ; \operatorname{Hom}(E, F)) \otimes_{\mathcal{C}^{\infty}(X)} \operatorname{Diff}_{\mathrm{sc}}^{m}(X) .
$$

This just means that $P \in \operatorname{Diff}_{\mathrm{sc}}^{m}(X ; E, F)$ is a local operator, $P: \mathcal{C}^{\infty}(X ; E) \longrightarrow$ $\mathcal{C}^{\infty}(X ; F)$ which is such that if $e_{i}$ is a local basis for $E$ and $f_{j}$ is a local basis for $F$ over an open set $O \subset X$ and $K \Subset O$ is compact there exist elements $P_{i j} \in \operatorname{Diff}_{\mathrm{sc}}^{m}(X)$ such that

$$
P\left(\phi e_{i}\right)=\sum_{j}\left(P_{i j} \phi\right) f_{j}, \forall \phi \in \mathcal{C}_{c}^{\infty}(O) \text { with } \operatorname{supp}(\phi) \subset K .
$$

Proposition 1. On any manifold with boundary the exterior differential extends from the interior to define $d \in \operatorname{Diff}_{\mathrm{sc}}^{1}\left(X ;{ }^{\mathrm{sc}} \Lambda^{k},{ }^{\mathrm{sc}} \Lambda^{k+1}\right)$ and $d=x A$ with $A \in \operatorname{Diff}_{\mathrm{b}}^{1}\left(X ;{ }^{\mathrm{sc}} \Lambda^{k},{ }^{\mathrm{sc}} \Lambda^{k+1}\right)$. The adjoint of $d$ with respect to a scattering metric is an element $\operatorname{Diff}_{\mathrm{sc}}^{1}\left(X ;{ }^{\mathrm{sc}} \Lambda^{k},{ }^{\mathrm{sc}} \Lambda^{k-1}\right)$ for each $k$ and is similarly of the form $x B$ with $B \in \operatorname{Diff}_{\mathrm{b}}^{1}\left(X ;{ }^{\mathrm{sc}} \Lambda^{k},{ }^{\mathrm{sc}} \Lambda^{k-1}\right)$. 
Proof: The statements concerning $d$ follow by explicit computation. Thus, in terms of the basis given by $(3.20)$

$$
\begin{gathered}
d\left(a \frac{d x}{x^{2}} \wedge \frac{d y^{\alpha}}{x^{k-1}}\right)=x\left(-\sum_{j} \frac{\partial a}{\partial y_{j}} \frac{d x}{x^{2}} \wedge \frac{d y_{j}}{x} \wedge \frac{d y^{\alpha}}{x^{k-1}}\right) \\
d\left(b \frac{d y^{\beta}}{x^{k}}\right)=x\left(\left[x \frac{\partial b}{\partial x}-k b\right] \frac{d x}{x^{2}} \wedge \frac{d y^{\beta}}{x^{k}}-\sum_{j} x \frac{\partial b}{\partial y_{j}} \frac{d y_{j}}{x} \wedge \frac{d y^{\beta}}{x^{k}}\right)
\end{gathered}
$$

from which it follows that $d=x A$ with $A \in \operatorname{Diff}_{\mathrm{b}}^{1}\left(X ;{ }^{\mathrm{sc}} \Lambda^{k},{ }^{\mathrm{sc}} \Lambda^{k+1}\right)$.

The Riemannian measure is of the form $x^{-n-1} v$, with $v$ a smooth non-vanishing measure. From this it follows that the adjoint of $d=x A$ is $\delta=A^{*} x$ with $A^{*} \in$ $\operatorname{Diff}_{\mathrm{b}}^{1}\left(X ;{ }^{\mathrm{sc}} \Lambda^{k},{ }^{\mathrm{sc}} \Lambda^{k-1}\right)$. Since $x^{r} \operatorname{Diff}_{\mathrm{b}}^{1}(X ; E, F) x^{-r}=\operatorname{Diff}_{\mathrm{b}}^{1}(X ; E, F)$ for any vector bundles the statements about $\delta$ also follow.

Notice that this result implies that the Laplacian of a scattering metric, acting on scattering forms, is again short range:

$$
\Delta \in \operatorname{Diff}_{\mathrm{sc}}^{1}\left(X ;{ }^{\mathrm{sc}} \Lambda^{k}\right)=x B_{k} x, B_{k} \in \operatorname{Diff}_{\mathrm{b}}^{2}\left(X ;{ }^{\mathrm{sc}} \Lambda^{k}\right) .
$$

This is not true if the bundles ${ }^{\mathrm{sc}} \Lambda^{*} X$ are replaced by the ordinary form bundles $\Lambda^{*} X$.

\section{Section 4: The scattering calculus defined.}

The (small) calculus of scattering pseudodifferential operators on a compact manifold with boundary is very closely related to familiar spaces of pseudodifferential operators on $\mathbb{R}^{n}$, essentially going back to Kohn and Nirenberg [1] and Hörmander [2]. This enables us to give an elementary, if somewhat clumsy, definition. In Appendix $\mathrm{B}$ this definition is replaced by one which is more geometric and intrinsic. This latter definition fits into the general scheme of [8] and so has the advantage that it can be integrated into other settings.

Consider the space of operators on $\mathcal{S}\left(\mathbb{R}^{n}\right)$ arising in Hörmander's formulation of the Weyl calculus [5] for the metric

$$
\frac{|d z|^{2}}{1+|z|^{2}}+\frac{|d \zeta|^{2}}{1+|\zeta|^{2}}
$$

These operators have Schwartz kernels of the (left-reduced) form

$$
A^{\prime}\left(z, z^{\prime}\right)=\frac{1}{(2 \pi)^{n}} \int e^{i\left(z-z^{\prime}\right) \cdot \zeta} a_{L}(z, \zeta) d \zeta
$$


where the amplitude satisfies the estimates

$$
\left|D_{z}^{\alpha} D_{\zeta}^{\beta} a_{L}(z, \zeta)\right| \leq C_{\alpha \beta}(1+|z|)^{-l-|\alpha|}(1+|\zeta|)^{m-|\beta|}
$$

the weight function being $(1+|z|)^{-l}(1+|\zeta|)^{m}$.

We shall use stereographic projection, (1.1), to transfer these operators to $\mathbb{S}_{+}^{n}$. Clearly

$$
\mathrm{SP}^{*}: \dot{\mathcal{C}}^{\infty}\left(\mathbb{S}_{+}^{n}\right) \longrightarrow \mathcal{S}\left(\mathbb{R}^{n}\right)
$$

is a isomorphism, with $\dot{\mathcal{C}}^{\infty}(X)=\bigcap_{k} x^{k} \mathcal{C}^{\infty}(X)$ for any compact manifold with boundary, $X$, and boundary defining function $x$.

Definition 1. The space $\Psi_{\mathrm{scc}}^{m, l}\left(\mathbb{S}_{+}^{n}\right)$ consists of those operators $A: \dot{\mathcal{C}}^{\infty}\left(\mathbb{S}_{+}^{n}\right) \longrightarrow$ $\dot{\mathcal{C}}^{\infty}\left(\mathbb{S}_{+}^{n}\right)$ such that if $A^{\prime}$ is defined by

$$
\mathrm{SP}^{*}(A \varphi)=A^{\prime}\left(\mathrm{SP}^{*} \varphi\right) \quad \forall \varphi \in \dot{\mathcal{C}}^{\infty}\left(\mathbb{S}_{+}^{n}\right)
$$

then $A^{\prime}$ is of the form (4.1), (4.2)

The subscript 'scc' stands for scattering-conormal; these spaces are refined below.

Applied in each factor stereographic projection also gives a compactification

$$
\mathrm{SP}_{2}: \mathbb{R}^{n} \times \mathbb{R}^{n} \longrightarrow \mathbb{S}_{+}^{n} \times \mathbb{S}_{+}^{n} .
$$

Let $\rho_{N}$ and $\rho_{\sigma}$ be the lifts to $\mathbb{S}_{+}^{n} \times \mathbb{S}_{+}^{n}$ of defining functions in the two factors; for example one can take

$$
\mathrm{SP}^{*} \rho_{N}=\left(1+|z|^{2}\right)^{-\frac{1}{2}} \text { and } \mathrm{SP}^{*} \rho_{\sigma}=\left(1+|\zeta|^{2}\right)^{-\frac{1}{2}} .
$$

Let $\mathcal{V}_{\mathrm{b}}\left(\mathbb{S}_{+}^{n} \times \mathbb{S}_{+}^{n}\right)$ be the Lie algebra of smooth vector fields which are tangent to both boundaries and let Diff* $\left(\mathbb{S}_{+}^{n} \times \mathbb{S}_{+}^{n}\right)$ be its enveloping algebra. For any $l, m \in \mathbb{R}$ define

$$
\begin{aligned}
\mathcal{A}^{m, l}\left(\mathbb{S}_{+}^{n} \times \mathbb{S}_{+}^{n}\right)=\left\{u \in \rho_{N}^{l} \rho_{\sigma}^{-m} L^{\infty}\left(\mathbb{S}_{+}^{n} \times \mathbb{S}_{+}^{n}\right) ;\right. \\
\left.\quad \operatorname{Diff}_{\mathrm{b}}^{*}\left(\mathbb{S}_{+}^{n} \times \mathbb{S}_{+}^{n}\right) u \subset \rho_{N}^{l} \rho_{\sigma}^{-m} L^{\infty}\left(\mathbb{S}_{+}^{n} \times \mathbb{S}_{+}^{n}\right)\right\} .
\end{aligned}
$$

These are the $L^{\infty}$-based conormal spaces of multi-order $(m, l)$. In fact, under $\mathrm{SP}_{2}^{*}$, the estimates in (4.5) correspond exactly to those in (4.2) in the obvious sense that

$$
a_{L} \text { satisfies }(4.2) \Longleftrightarrow a_{L} \in \mathrm{SP}_{2}^{*} \mathcal{A}^{m, l}\left(\mathbb{S}_{+}^{n} \times \mathbb{S}_{+}^{n}\right) .
$$

We then fix a ('classical') subspace of the scattering-conormal pseudodifferential operators on $\mathbb{S}_{+}^{n}$ by: 
DEFINITION 2. The space $\Psi_{\mathrm{sc}}^{m, l}\left(\mathbb{S}_{+}^{n}\right) \subset \Psi_{\mathrm{scc}}^{m, l}\left(\mathbb{S}_{+}^{n}\right)$ consists of those operators for which

$$
a_{L} \in \mathrm{SP}^{*}\left(\rho_{N}^{l} \rho_{\sigma}^{-m} \mathcal{C}^{\infty}\left(\mathbb{S}_{+}^{n} \times \mathbb{S}_{+}^{n}\right)\right)
$$

For notational convenience below we shall let 'sk' stand for either 'sc' or 'scc' but fixed throughout a particular discussion.

To transfer this definition to a general compact manifold with boundary we invoke (but for the moment do not actually use) the coordinate invariance of these spaces. Thus if $F: \mathbb{S}_{+}^{n} \longrightarrow \mathbb{S}_{+}^{n}$ is a diffeomorphism then

$$
\Psi_{\mathrm{sk}}^{m, l}\left(\mathbb{S}_{+}^{n}\right) \ni A \longmapsto\left(F^{-1}\right)^{*} \cdot A \cdot F^{*} \in \Psi_{\mathrm{sk}}^{m, l}\left(\mathbb{S}_{+}^{n}\right)
$$

is an automorphism. This can be checked directly and in any case follows from the discussion in Appendix B.

The Schwartz kernel theorem realizes the kernel of an operator $A: \dot{\mathcal{C}}^{\infty}(X) \longrightarrow$ $\dot{\mathcal{C}}^{\infty}(X)$, for $X$ a compact manifold with boundary, as an (extendible) distribution which we write as $A \in \mathcal{C}^{-\infty}\left(X^{2} ; \pi_{R}^{*} \Omega\right)$ where $\pi_{R}: X^{2} \longrightarrow X$ is the right projection and $\Omega$ is the density bundle on $X$. It is easy to see that the 'residual spaces'

$$
\Psi_{\mathrm{sk}}^{-\infty, \infty}\left(\mathbb{S}_{+}^{n}\right) \equiv \dot{\mathcal{C}}^{\infty}\left(\mathbb{S}_{+}^{n} \times \mathbb{S}_{+}^{n} ; \pi_{R}^{*} \Omega\right)
$$

correspond to smooth kernels vanishing to all orders of both boundaries. In the general case we simply define

$$
\Psi_{\mathrm{sk}}^{-\infty, \infty}(X)=\dot{\mathcal{C}}^{\infty}\left(X^{2} ; \pi_{R}^{*} \Omega\right)
$$

To define the full calculus we use a covering of $X$ by coordinate systems $F: O \longleftrightarrow$ $O^{\prime} \subset \mathbb{S}_{+}^{n}$ where $O \subset X$ and $O^{\prime}$ are relatively open sets and $F$ is a diffeomorphism of manifolds with boundary.

Definition 3. If $X$ is a compact manifold with boundary and $m, l \in \mathbb{R}$ the spaces $\Psi_{\mathrm{sk}}^{m, l}(X)$, for sk $=\mathrm{sc}$ or scc, consists of those operators $A: \dot{\mathcal{C}}^{\infty}(X) \longrightarrow \dot{\mathcal{C}}^{\infty}(X)$ such that if $\varphi, \psi \in \mathcal{C}^{\infty}(X)$ have disjoint supports then $\varphi A \psi \in \Psi_{\mathrm{sk}}^{-\infty, \infty}(X)$ and if $F: O \longrightarrow O^{\prime} \subset \mathbb{S}_{+}^{n}$ is a coordinates patch with $\varphi \in \mathcal{C}^{\infty}(X), \psi \in \mathcal{C}^{\infty}\left(\mathbb{S}_{+}^{n}\right)$ having $\operatorname{supp}(\varphi) \subset O, \operatorname{supp}(\psi) \subset O^{\prime}$ then

$$
A_{F, \psi, \varphi}=\left(F^{-1}\right)^{*} \circ \psi \cdot A \circ F^{*} \circ \varphi \cdot \in \Psi_{\mathrm{sk}}^{m, l}\left(\mathbb{S}_{+}^{n}\right) .
$$

The properties of the calculus described in the next section can all be deduced directly from this definition and the results of [5]; alternate more global arguments are outlined in Appendix B. The model case of $\mathbb{S}_{+}^{n}$ has one particularly useful property, namely invariance under Fourier transformation. 
Proposition 2. Let $\mathcal{F}: \mathcal{S}\left(\mathbb{R}^{n}\right) \longrightarrow \mathcal{S}\left(\mathbb{R}^{n}\right)$ be the Fourier transform

$$
\mathcal{F} \varphi(\zeta)=\int e^{-i z \cdot \zeta} \varphi(z) d z
$$

and $\overline{\mathcal{F}}: \dot{\mathcal{C}}^{\infty}\left(\mathbb{S}_{+}^{n}\right) \longrightarrow \dot{\mathcal{C}}^{\infty}\left(\mathbb{S}_{+}^{n}\right)$ be defined by

$$
\overline{\mathcal{F}}=\left(\mathrm{SP}^{*}\right)^{-1} \circ \mathcal{F} \circ \mathrm{SP}^{*}
$$

then

$$
\overline{\mathcal{F}} \circ \Psi_{\mathrm{sk}}^{m, l}\left(\mathbb{S}_{+}^{n}\right) \circ \overline{\mathcal{F}}^{-1}=\Psi_{\mathrm{sk}}^{-l,-m}\left(\mathbb{S}_{+}^{n}\right) .
$$

Proof: The action of the operator in (4.1), (4.2) can be written

$$
A^{\prime} \varphi(z)=\frac{1}{2 \pi)^{n}} \int e^{i z \cdot \zeta} a_{L}(z, \zeta)(\mathcal{F} \varphi)(\zeta) d \zeta .
$$

Thus if $A^{\prime \prime}=\mathcal{F} \circ A^{\prime} \circ \mathcal{F}^{-1}$ then

$$
A^{\prime \prime} \psi(z)=\frac{1}{(2 \pi)^{2}} \int e^{i\left(z^{\prime}-z\right) \cdot \zeta} a_{L}\left(\zeta, z^{\prime}\right) \psi\left(z^{\prime}\right) d z^{\prime} d \zeta .
$$

That this can again be written in the form (4.1), (4.2) is just the equivalence of left- and right-reduced forms for this calculus.

\section{Section 5: Properties of the calculus.}

Next various of the properties of the calculus of operators defined above are described. The most important property is that the spaces form a bi-filtered algebra:

$$
\Psi_{\mathrm{sk}}^{m_{1}, l_{1}}(X) \circ \Psi_{\mathrm{sk}}^{m_{2}, l_{2}}(X) \subset \Psi_{\mathrm{sk}}^{m_{1}+m_{2}, l_{1}+l_{2}}(X) .
$$

Furthermore they do indeed contain the scattering differential operators

$$
\operatorname{Diff}_{\mathrm{sc}}^{m}(X) \subset \Psi_{\mathrm{sc}}^{m, 0}(X), m \in \mathbb{N}_{0} .
$$

There is also a basic conjugation-invariance under multiplication by powers of a boundary defining functions $x \in \mathcal{C}^{\infty}(X)$ :

$$
x^{L} \cdot \Psi_{\mathrm{sk}}^{m, l}(X)=\Psi_{\mathrm{sk}}^{m, l}(X) \cdot x^{L}=\Psi_{\mathrm{sc}}^{m, l+L}(X) .
$$

As for the standard calculus on a compact manifold without boundary the main computational tool is the symbol mapping; in this case it has two parts just as it does for the scattering differential operators. 


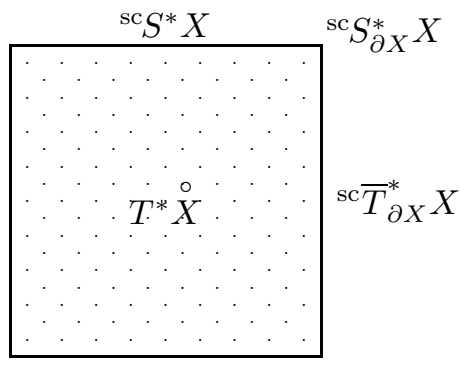

Figure 1. Boundary faces of ${ }^{\mathrm{sc}} \bar{T}^{*} X$.

Let ${ }^{\mathrm{sc}} \bar{T}^{*} X$ be the compact manifold with corners of codimension two obtained by radial compactification of the fibres of ${ }^{\mathrm{sc}} T^{*} X$, as discussed in $\S 1$. We shall denote its boundary by

$$
C_{\mathrm{sc}} X=\partial\left({ }^{\mathrm{sc}} \bar{T}^{*} X\right) .
$$

This is a topological but not, in any natural way, a $\mathcal{C}^{\infty}$ manifold without boundary. Indeed it consists of two smooth manifolds with boundary

$$
C_{\mathrm{sc}} X={ }^{\mathrm{sc}} S^{*} X \cup{ }^{\mathrm{sc}} \bar{T}_{\partial X}^{*} X
$$

which meet at their boundaries, with a natural identification.

$$
{ }^{\mathrm{sc}} S^{*} X \cap{ }^{\mathrm{sc}} \bar{T}_{\partial X}^{*} X={ }^{\mathrm{sc}} S_{\partial X}^{*} X .
$$

Here ${ }^{\mathrm{sc}} S_{p}^{*} X$ is the sphere

$$
{ }^{\mathrm{sc}} S_{p}^{*} X=\left({ }^{\mathrm{sc}} T_{p}^{*} X \backslash 0\right) / \mathbb{R}^{+}
$$

which is the sphere at infinity of the radial compactification of the fibre. We shall use the notation

$$
\begin{aligned}
& \mathcal{C}^{\infty}\left(C_{\mathrm{sc}} X\right)=\left\{\left(u_{\sigma}, u_{N}\right) \in \mathcal{C}^{\infty}\left({ }^{\mathrm{sc}} S^{*} X\right)\right. \times \mathcal{C}^{\infty}\left({ }^{\mathrm{sc}} \bar{T}_{\partial X}^{*} X\right) ; \\
&\left.\left.\left(u_{\sigma}\right)\right|_{\Gamma^{\mathrm{sc}} S_{\partial X}^{*} X}=\left.\left(u_{N}\right)\right|_{\mid \mathrm{sc}} S_{\partial X}^{*} X\right\} .
\end{aligned}
$$

More generally if $E$ is a vector bundle over ${ }^{\mathrm{sc}} \bar{T}^{*} X$ then

$$
\begin{aligned}
\mathcal{C}^{\infty}\left(C_{\mathrm{sc}} X ; E\right)=\left\{\left(u_{\sigma}, u_{N}\right) \in \mathcal{C}^{\infty}\left({ }^{\mathrm{sc}} S^{*} X ; E\right) \times \mathcal{C}^{\infty}\left({ }^{\mathrm{sc}} \bar{T}_{\partial X}^{*} X ; E\right) ;\right. \\
\left.\left(u_{\sigma}\right) \mid{ }^{\mathrm{sc}} S_{\partial X}^{*} X=\left(u_{N}\right){ }^{\mathrm{sc}} S_{\partial X}^{*} X\right\} .
\end{aligned}
$$


In fact this is well-defined if $E_{\sigma}$ and $E_{N}$ are vector bundles over ${ }^{\mathrm{sc}} S^{*} X$ and ${ }^{\mathrm{sc}} \bar{T}_{\partial X}^{*} X$ respectively, with an identification specified of their restrictions to ${ }^{\mathrm{sc}} S_{\partial X}^{*} X$. For any $m, l \in \mathbb{R}$ the space

$$
\rho_{N}^{l} \rho_{\sigma}^{-m} \mathcal{C}^{\infty}\left({ }^{\mathrm{sc}} \bar{T}^{*} X\right)
$$

is the space of all sections of a trivial bundle, which we shall denote $S^{m, l}$, over ${ }^{\text {sc }} \bar{T}^{*} X$. Directly from the definition, (5.8), the bundles $S^{m, l}$ all have b-connections, i.e. we can identify $V a$ as a section of $S^{m, l}$ whenever $V \in \mathcal{V}_{\mathrm{b}}\left({ }^{\mathrm{sc}} \bar{T}^{*} X\right)$ is a smooth vector field tangent to both boundaries and $a$ is a smooth section of $S^{m, l}$.

Proposition 3. For any compact manifold with boundary, $X$, and any vector bundles $E$ and $F$ over $X$ the combined symbol map

$$
j_{\mathrm{sc}, m, l}(A)=\left(\sigma_{\mathrm{sc}, m}(A), \widehat{N}_{\mathrm{sc}}(A)\right)
$$

gives an exact sequence

$$
\begin{aligned}
0 \longrightarrow \Psi_{\mathrm{sc}}^{m-1, l+1}(X ; E, F) \longrightarrow & \Psi_{\mathrm{sc}}^{m, l}(X ; E, F) \\
& \stackrel{j_{\mathrm{sc}, m, l}}{\longrightarrow} \mathcal{C}^{\infty}\left(C_{\mathrm{sc}} X ; S^{m, l} \otimes \operatorname{Hom}_{\partial X}(E, F)\right) \longrightarrow 0 .
\end{aligned}
$$

There is a similar result for the larger calculus with a more complicated image space. Extending the definition (4.5) set

$$
\begin{aligned}
\mathcal{A}^{m, l}\left({ }^{\mathrm{sc}} \bar{T}^{*} X\right)=\left\{u \in \rho_{N}^{l} \rho_{\sigma}^{-m} L^{\infty}\left({ }^{\mathrm{sc}} \bar{T}^{*} X\right) ;\right. & \\
& \left.\operatorname{Diff}_{\mathrm{b}}^{*}\left({ }^{\mathrm{sc}} \bar{T}^{*} X\right) u \subset \rho_{N}^{l} \rho_{\sigma}^{-m} L^{\infty}\left({ }^{\mathrm{sc}} \bar{T}^{*} X\right)\right\}
\end{aligned}
$$

where now $\rho_{N}$ is the lift from $X$ of a boundary defining function and $\rho_{\sigma}$ defines the other, fibre, boundaries. Let us denote the quotient by

$$
\mathcal{A}^{\{m, l\}}\left(C_{\mathrm{sc}} X\right)=\mathcal{A}^{m, l}\left({ }^{\mathrm{sc}} \bar{T}^{*} X\right) / \mathcal{A}^{m-1, l+1}\left({ }^{\mathrm{sc}} T^{*} X\right)
$$

where the notation is justified by the fact that it is at least a sheaf over $C_{\mathrm{sc}} X$. Notice that

$$
\mathcal{C}^{\infty}\left({ }^{\mathrm{sc}} \bar{T}^{*} X ; S^{m, l}\right) \subset \mathcal{A}^{m, l}\left({ }^{\mathrm{sc}} \bar{T}^{*} X\right)
$$

and that in a natural way $\mathcal{C}^{\infty}\left(C_{\mathrm{sc}} X ; S^{m, l}\right) \subset \mathcal{A}^{\{m, l\}}\left(C_{\mathrm{sc}} X\right)$. Proposition 3 is replaced for the scattering-conormal calculus by a short exact sequence

$$
0 \longrightarrow \Psi_{\mathrm{scc}}^{m-1, l+1}(X) \longrightarrow \Psi_{\mathrm{scc}}^{m, l}(X) \stackrel{j_{\mathrm{sc}, m, l}}{\longrightarrow} \mathcal{A}^{\{m, l\}}\left(C_{\mathrm{sc}} X\right) \longrightarrow 0
$$

and similarly for operators on sections of vector bundles.

In both cases the symbol maps are multiplicative:

$$
\begin{gathered}
j_{\mathrm{sc}, m_{1}+m_{2}, l_{1}+l_{2}}(A \cdot B)=j_{\mathrm{sc}, m_{1}, l_{1}}(A) \cdot j_{\mathrm{sc}, m_{2}, l_{2}}(B) \\
A \in \Psi_{\mathrm{sk}}^{m_{1}, l_{1}}(X), B \in \Psi_{\mathrm{sk}}^{m_{2}, l_{2}}(X),
\end{gathered}
$$

with values in commutative algebras. For use in propagation theorems it is important to have a formula for the (joint) symbol of a commutator. Recall that the joint symbol is defined on the boundary of ${ }^{\mathrm{sc}} \bar{T}^{*} X$, the interior of which is canonically identified with the interior of $T^{*} X$ and then: 
Proposition 4. The Poisson bracket on $T^{*} X$ extends by continuity from the interior of ${ }^{\mathrm{sc}} \bar{T}^{*} X$ to define

$$
\begin{gathered}
\{,\}: \mathcal{C}^{\infty}\left(C_{\mathrm{sc}} X ; S^{m_{1}, l_{1}}\right) \times \mathcal{C}^{\infty}\left(C_{\mathrm{sc}} X ; S^{m_{2}, l_{2}}\right) \longrightarrow \mathcal{C}^{\infty}\left(C_{\mathrm{sc}} X ; S^{m_{1}+m_{2}, l_{1}+l_{2}}\right) \\
\{,\}: \mathcal{A}^{\left\{m_{1}, l_{1}\right\}}\left(C_{\mathrm{sc}} X\right) \times \mathcal{A}^{\left\{m_{2}, l_{2}\right\}}\left(C_{\mathrm{sc}} X\right) \longrightarrow \mathcal{A}^{\left\{m_{1}+m_{2}, l_{1}+l_{2}\right\}}\left(C_{\mathrm{sc}} X\right)
\end{gathered}
$$

and for any $m_{1}, m_{2}, l_{1}, l_{2} \in \mathbb{R}$

$$
\begin{gathered}
j_{\mathrm{sc}, m_{1}+m_{2}, l_{1}+l_{2}}([A, B])=\frac{1}{i}\left\{j_{\mathrm{sc}, m_{1}, l_{1}}(A), j_{\mathrm{sc}, m_{2}, l_{2}}(B)\right\} \\
\forall A \in \Psi_{\mathrm{sk}}^{m_{1}, l_{1}}(X), B \in \Psi_{\mathrm{sk}}^{m_{2}, l_{2}}(X) .
\end{gathered}
$$

Proof: This follows from the symbol calculus of [5] and the local definition of the scattering calculus.

Naturally we want an explicit formula for this bracket, especially near the boundary. Let us first note what we need to compute:

Lemma 5. If $a \in \mathcal{C}^{\infty}\left({ }^{\mathrm{sc}} \bar{T}^{*} X ; S^{m, l}\right)$ the Hamilton vector field extends from the interior to be of the form

$$
H_{a}=\rho_{\sigma}^{-m+1} \rho_{N}^{l+1 \mathrm{sc}} H_{a}^{m, l},{ }^{\mathrm{sc}} H_{a}^{m, l} \in \mathcal{V}_{\mathrm{b}}\left({ }^{\mathrm{sc}} \bar{T}^{*} X\right)
$$

and for any $b \in \mathcal{A}^{\left\{m^{\prime}, l^{\prime}\right\}}\left({ }^{\mathrm{sc}} \bar{T}^{*} X\right)$

$$
\{a, b\}=\rho_{\sigma}^{-m+1} \rho_{N}^{l+1 \mathrm{sc}} H_{a}^{m, l} b \in \mathcal{A}^{\left\{m+m^{\prime}-1, l+l^{\prime}+1\right\}}\left({ }^{\mathrm{sc}} \bar{T}^{*} X\right)
$$

depends on a only through the indicial operator $I\left({ }^{\mathrm{sc}} H_{a}^{m, l}\right) \in \mathcal{C}^{\infty}\left(C_{\mathrm{sc}} ;{ }^{\mathrm{b}} T\left({ }^{\mathrm{sc}} \bar{T}^{*} X\right)\right)$.

Note that, for $a \in \mathcal{C}^{\infty}\left(C_{\mathrm{sc}} ;{ }^{\mathrm{b}} T\left({ }^{\mathrm{sc}} \bar{T}^{*} X\right)\right)$, the vector field ${ }^{\mathrm{sc}} H_{a}^{m, l}$ is not well defined, since it depends on the choices of $\rho_{\sigma}$ and $\rho_{N}$. However different choices effect the multiplication of ${ }^{\mathrm{sc}} H_{a}^{m, l}$ by a positive smooth factor, so this is seldom significant. In any case the metric (1) fixes a choice of $\rho_{\sigma}$ and $\rho_{N}$ up to quadratic terms:

$$
\rho_{\sigma}=\left(1+|\zeta|^{2}\right)^{-\frac{1}{2}}, \rho_{N}=x
$$

so we can regard ${ }^{\mathrm{sc}} H_{a}^{m, l} \in \mathcal{C}^{\infty}\left(C_{\mathrm{sc}} ;{ }^{\mathrm{b}} T\left({ }^{\mathrm{sc}} \bar{T}^{*} X\right)\right)$ as well defined.

We proceed to compute the form of this normalized Hamilton vector field at a general boundary point in terms of coordinates $x, y_{1}, \ldots, y_{n-1}$ where $x$ is a local defining function for the boundary. Let $\xi, \eta_{1}, \ldots, \eta_{n-1}$ be the canonically dual coordinates in $T^{*} X$. The singular forms $d x / x^{2}, d y_{j} / x$ give a basis for ${ }^{\mathrm{sc}} T^{*} X$ locally so 'canonically dual' coordinates in ${ }^{\text {sc }} T^{*} X$ arise as the coefficients, $\tau, \mu_{1}, \ldots, \mu_{n-1}$ of the form. Thus the identification of $T^{*} X$ and ${ }^{{ }^{\mathrm{sc}}} T^{*} X$ in $x>0$ is just

$$
\tau=x^{2} \xi, \mu=x \eta
$$


An element of $S^{m_{1}, l_{1}}$, or $\mathcal{A}^{\left\{m, l_{1}\right\}}$, is locally of the form

$$
a=x^{l_{1}} \alpha(x, y, \tau, \mu)
$$

with $\alpha$ bounded. The Poisson bracket of $a$ and $b=x^{l_{2}} \beta$ can be written

$$
\{a, b\}=\frac{\partial x^{l_{1}} \alpha}{\partial \xi} \frac{\partial x^{l_{2}} \beta}{\partial x}-\frac{\partial x^{l_{1}} \alpha}{\partial x} \frac{\partial x^{l_{2}} \beta}{\partial \xi}+\sum_{j=1}^{n-1}\left(\frac{\partial x^{l_{1}} \alpha}{\partial \eta_{j}} \frac{\partial x^{l_{2}} \beta}{\partial y_{j}}-\frac{\partial x^{l_{1}} \alpha}{\partial y_{j}} \frac{\partial x^{l_{2}} \beta}{\partial \eta_{j}}\right)
$$

Making the substitution (5.20) reduces this to

$$
\begin{aligned}
x^{l_{1}+l_{2}+1}\left(\frac { \partial \alpha } { \partial \tau } \left(x \frac{\partial \beta}{\partial x}+\right.\right. & \left.R_{\mu} \beta+l_{2} \beta\right)-\left(x \frac{\partial \alpha}{\partial x}+R_{\mu} \alpha+l_{1} a\right) \frac{\partial \beta}{\partial \tau} \\
& \left.+\sum_{j=1}^{n-1}\left(\frac{\partial \alpha}{\partial \eta_{j}} \frac{\partial \beta}{\partial y_{j}}-\frac{\partial \alpha}{\partial y_{j}} \frac{\partial \beta}{\partial \eta_{j}}\right)\right)+O\left(x^{l_{1}+l_{2}+2}\right)
\end{aligned}
$$

so at least near ${ }^{\mathrm{sc}} T_{\partial x}^{*} X$ we find

$$
\begin{aligned}
{ }^{\mathrm{sc}} H_{a}^{*, l_{1}}=\left(\frac{\partial \alpha}{\partial \tau}\left(x \frac{\partial}{\partial x}+R_{\mu}\right)\right. & -\left(x \frac{\partial \alpha}{\partial x}+R_{\mu} \alpha+l_{1} \alpha\right) \frac{\partial}{\partial \tau} \\
& \left.+\sum_{j+1}^{n-1}\left(\frac{\partial \alpha}{\partial \eta_{j}} \frac{\partial}{\partial y_{j}}-\frac{\partial \alpha}{\partial y_{j}} \frac{\partial}{\partial \eta_{j}}\right)\right)
\end{aligned}
$$

when $R_{\mu}=\mu \cdot \partial / \partial_{\mu}$ is the $\mu$-radial vector field.

Then if $b=x^{l_{2}} \beta \in \mathcal{A}^{*, l_{2}}\left({ }^{\mathrm{sc}} \bar{T}^{*} X\right)$ near ${ }^{\mathrm{sc}} T_{\partial X}^{*} X$

$$
\{a, b\}=x^{l_{1}+1 \mathrm{sc}} H_{a}^{*, l_{1}} b=x^{l_{1}+l_{2}+1}\left({ }^{\mathrm{sc}} H_{a}^{*, l_{1}} \beta+l_{2} \frac{\partial \alpha}{\partial \tau} \beta\right) .
$$

Similar formulæ can easily be obtained near the other boundary faces of ${ }^{\mathrm{sc}} \bar{T}^{*} X$.

The boundedness of scattering pseudodifferential operators on $L^{2}$-based Sobolev spaces also follows from the work of Hörmander [5]. Let $L_{\mathrm{sc}}^{2}(X)$ be the metric $L^{2}$ space for a scattering metric

$$
L_{\mathrm{sc}}^{2}(X)=\left\{u \in L_{\mathrm{loc}}^{2}(\stackrel{\circ}{X}) ; \int_{X}|u|^{2} d g<\infty\right\} .
$$

For $X=\mathbb{S}_{+}^{n}$ this is just the image of the Euclidian $L^{2}$ space under stereographic projection. For non-negative integers $m$, and $l \in \mathbb{R}$ set

$$
H_{\mathrm{sc}}^{m, l}(X)=\left\{u \in \mathcal{C}^{\infty}(X) ; \operatorname{Diff}_{\mathrm{sc}}^{m}(X) u \subset x^{l} L_{\mathrm{sc}}^{2}(X)\right\}
$$

and for general $m, l$ define

$$
H_{\mathrm{sc}}^{m, l}(X)=\left\{u \in \mathcal{C}^{-\infty}(X) ; \Psi_{\mathrm{sc}}^{m,-l}(X) u \subset L_{\mathrm{sc}}^{2}(X)\right\} .
$$

For the case of $\mathbb{S}_{+}^{n}, H_{\mathrm{sc}}^{m, 0}\left(\mathbb{S}_{+}^{n}\right)$ is the image under $\mathrm{SP}^{*}$ of the standard Sobolev space $H^{m}\left(\mathbb{R}^{n}\right)$. Naturally it needs to be checked that these definitions are consistent, that this is so corresponds to: 
Proposition 5. For any $m, l, M, L \in \mathbb{R}$ each $A \in \Psi_{\text {scc }}^{m, l}(X)$ defines a continuous linear operator

$$
A: H_{\mathrm{sc}}^{M, L}(X) \longrightarrow H_{\mathrm{sc}}^{M-m, L+l}(X) ;
$$

if $A \in \Psi_{\mathrm{sc}}^{m, l}(X)$ this map is compact if and only if $j_{\mathrm{sc}, m, l}(A) \equiv 0$.

In constructive arguments it is very useful to have asymptotic sums:

Lemma 6. If $A_{j} \in \Psi_{\mathrm{sk}}^{m-j, l}(X), B_{j} \in \Psi_{\mathrm{sk}}^{m, l+j}(X)$, and $C_{j} \in \Psi_{\mathrm{sk}}^{m-j, l+j}(X)$, for $j \in \mathbb{N}_{0}$, there exist asymptotic sums $A, B, C \in \Psi_{\mathrm{sk}}^{m, l}(X)$ in the sense that

$$
\begin{aligned}
& A-\sum_{j=0}^{N-1} A_{j} \in \Psi_{\mathrm{sk}}^{m-N, l}(X) \\
& B-\sum_{j=0}^{N-1} B_{j} \in \Psi_{\mathrm{sk}}^{m, l+N}(X) \\
& C-\sum_{j=0}^{N-1} C_{j} \in \Psi_{\mathrm{sk}}^{m-N, l+N}(X)
\end{aligned}
$$

and the sums are determined uniquely modulo $\Psi_{\mathrm{sk}}^{-\infty, l}(X), \Psi_{\mathrm{sk}}^{m, \infty}(X)$ and $\Psi_{\mathrm{sc}}^{-\infty, \infty}(X)$ respectively.

The spaces $\Psi_{\mathrm{sk}}^{m, l}(X)$ have natural complete locally convex topologies in which $\Psi_{\mathrm{sc}}^{-\infty, \infty}(X)$ is strongly dense. Thus any $A \in \Psi_{\mathrm{sk}}^{m, l}(X)$ can be approximated by $A_{j} \in \Psi_{\mathrm{sc}}^{-\infty, \infty}(X)$ in the topology of $\Psi_{\mathrm{scc}}^{m+\delta, l-\delta}(F)$ for any $\delta>0$ and such that $A_{j}$ is bounded in $\Psi_{\mathrm{scc}}^{m, l}(X)$ with

$$
A_{j} \longrightarrow A \text { in the strong operator topology for (5.28) }
$$

This approximability is closely related to the existence of quantization maps. There is no 'natural' quantization map but there does exist a continuous linear map

$$
\begin{aligned}
& q: \mathcal{A}^{\infty,-\infty}\left({ }^{\mathrm{sc}} \bar{T}^{*} X\right) \longrightarrow \Psi_{\mathrm{scc}}^{\infty,-\infty}(X) \\
& q\left(\mathcal{A}^{m, l}\left(\mathrm{sc}^{*} \bar{T}^{*} X\right)\right) \subset \Psi_{\mathrm{scc}}^{m, l}(X) \forall m, l
\end{aligned}
$$

which is continuous and linear (not multiplicative) and such that

$$
q\left(\mathcal{C}^{\infty}\left({ }^{\mathrm{sc}} \bar{T}^{*} X ; S^{m, l}\right)\right) \subset \Psi_{\mathrm{sc}}^{m, l}(X) .
$$


It can also be taken to project to a left inverse to the symbol map, in the sense that

$$
j_{\mathrm{sc}, m, l} \circ q: \mathcal{A}^{m, l}\left({ }^{\mathrm{sc}} \bar{T}^{*} X\right) \longrightarrow \mathcal{A}^{\{m, l\}}\left(C_{\mathrm{sc}} X\right)
$$

is the natural projection.

The residual space $\Psi_{\mathrm{sc}}^{-\infty, \infty}(X)$ is 'highly ignorable' in the sense that it is a bi-ideal in the space of all operators on $X$. Thus

$$
\Psi_{\mathrm{sc}}^{-\infty, \infty}(X) \ni A: \mathcal{C}^{-\infty}(X) \longrightarrow \dot{\mathcal{C}}^{\infty}(X)
$$

and if $Q: \dot{\mathcal{C}}^{\infty}(X) \longrightarrow \mathcal{C}^{-\infty}(X)$ is any continuous linear operator then

$$
A \circ Q \circ B \in \Psi_{\mathrm{sc}}^{-\infty, \infty}(X) \text { if } A, B \in \Psi_{\mathrm{sc}}^{-\infty, \infty}(X) .
$$

\section{Section 6: Resolvent family.}

Before turning to microlocal matters we illustrate the use of the global calculus.

THEOREM 1. If $\Delta$ is the Laplacian of a metric on a compact manifold with boundary, $X$, arising from a fibre metric on ${ }^{\mathrm{sc}} T X$ and $V \in \mathcal{C}^{\infty}(X)$ is real-valued then the resolvent family

$$
R(z)=(\Delta+V-z)^{-1}
$$

is a meromorphic function of $z \in \mathbb{C} \backslash\left[\inf _{\partial X} V, \infty\right)$ with values in $\Psi_{\mathrm{sc}}^{-2,0}(X)$ and with only simple poles, if any, in $\left[\inf _{X} V, \inf _{\partial X} V\right.$ ) with residues of finite rank in $\Psi_{\mathrm{sc}}^{-\infty, \infty}(X)$.

Proof: The symbol and normal operators of the Laplacian have already been computed in (3.1) and (3.2) (valid for general fibre metrics on ${ }^{\mathrm{sc}} T X$, not just those of the special form (1)). For $V \in \mathcal{C}^{\infty}(X)$ and $z \in \mathbb{C}$ the symbol of $(\Delta+V-z)$ is still $|\zeta|^{2}$ whereas the normal symbol is $|\zeta|^{2}+V(p)-z$ at $p \in \partial X$. If $z \notin\left[\inf _{\partial X} V, \infty\right)$ then

$$
\left(|\zeta|^{2}+V_{\uparrow \partial X}-z\right)^{-1} \in \mathcal{C}^{\infty}\left({ }^{\mathrm{sc}} \bar{T}_{\partial X}^{*} X ; S^{-2,0}\right)
$$

is compatible with $|\zeta|^{-2} \in \mathcal{C}^{\infty}\left({ }^{\mathrm{sc}} S^{*} X ; S^{-2,0}\right)$. Thus, from the exactness in (5.10) there exists $B_{0} \in \Psi_{\mathrm{sc}}^{-2,0}(X)$ satisfying

$$
\begin{gathered}
\widehat{N}_{\mathrm{sc}, p}\left(B_{0}\right)=\left(|\zeta|^{2}+V(p)-z\right)^{-1} \quad \forall p \in \partial X \\
\text { and } \sigma_{\mathrm{sc},-2}\left(B_{0}\right)=|\zeta|^{-2} .
\end{gathered}
$$

Then both $(\Delta+V-z) \cdot B_{0}$ and $B_{0} \cdot(\Delta+V-z)$ are elements of $\Psi_{\mathrm{sc}}^{0,0}(X)$ with joint symbol the same as that of the identity. Hence

$$
R_{1}=\mathrm{Id}-(\Delta+V-z) \circ B_{0} \in \Psi_{\mathrm{sc}}^{-1,1}(X) .
$$


The formal Neumann series for $\left(\operatorname{Id}-R_{1}\right)^{-1}$ :

$$
\mathrm{Id}+R_{1}+R_{1}^{2}+\cdots+R_{1}^{N}+\ldots
$$

has $N$ th term in $\Psi_{\mathrm{sc}}^{-N, N}(X)$ so, by Lemma 6 , can be summed asymptotically modulo $\Psi_{\mathrm{sc}}^{-\infty, \infty}(X)$ giving $B_{R}^{\prime}=B_{0} \circ\left(\mathrm{Id}+R^{\prime}\right) \in \Psi_{\mathrm{sc}}^{-2,0}(X)$ such that

$$
(\Delta+V-z) \cdot B_{R}^{\prime}=\mathrm{Id}-R_{\infty, R}, R_{\infty, R} \in \Psi_{\mathrm{sc}}^{-\infty, \infty}(X) .
$$

The same construction for $\bar{z}$ gives a left parametrix, $B_{L}^{\prime}$, in the same sense. Moreover

$(6.5)$

$B_{R}^{\prime}-B_{L}^{\prime} \equiv B_{L}^{\prime} \circ(\Delta+V-z) \circ B_{R}^{\prime}-B_{L}^{\prime} \circ(\Delta+V-z) \circ B_{R}^{\prime} \equiv 0$ modulo $\Psi_{\mathrm{sc}}^{-\infty, \infty}(X)$,

so either $B_{L}^{\prime}$ or $B_{R}^{\prime}$ gives a two-sided parametrix. In particular for these values of $z,(\Delta+V-z)$ is Fredholm as an operator from $H_{\mathrm{sc}}^{m, l}(X)$ to $H_{\mathrm{sc}}^{m-2, l}(X)$ for any $m$ or $l$.

The null space is in the range of $R_{\infty, L}$ so is contained in $\dot{\mathcal{C}}^{\infty}(X)$ and hence is independent of $m$ or $l$. As a closed subspace of $H_{\mathrm{sc}}^{0,0}(X)=L_{\mathrm{sc}}^{2}(X)$ it is necessarily finite dimensional. If $z \in \mathbb{C} \backslash\left[\inf _{X} V, \infty\right)$ integration by parts shows that there can be no such null space, so $(\Delta+V-z)^{-1}$ then exists. The identity

$$
(\Delta+V-z)^{-1}=B_{L}^{\prime}+B_{L}^{\prime} \circ R_{\infty, R}-R_{\infty, L} \circ(\Delta+V-z)^{-1} \circ R_{\infty, R}
$$

shows that $(\Delta+V-z)^{-1} \in \Psi_{\mathrm{sc}}^{-2,0}(X)$, by the bi-ideal property of the residual operators. Continuity in $z \in \mathbb{C} \backslash\left[\inf _{X} V, \infty\right)$, and hence holomorphy, follows directly.

Moreover the compactness of the error terms $R_{\infty, R}$ and $R_{\infty, L}$ as operators on $L_{\mathrm{sc}}^{2}(X)$ allows analytic Fredholm theory to be applied, so the resolvent extends to be meromorphic in $\mathbb{C} \backslash\left[\inf _{\partial X} V, \infty\right)$, where the parametrix is holomorphic. Any poles must be simple, in view of the self-adjointness and non-negativity of $\Delta+V$, with residue the orthogonal projection onto the null space.

No special properties of the Laplacian acting on functions have been used here, so the proof applies equally well to show that for the Laplacian on forms

$$
(\Delta-z)^{-1} \in \Psi_{\mathrm{sc}}^{-2,0}\left(X ;{ }^{\mathrm{sc}} \Lambda^{*}\right)
$$

is holomorphic in $z \in \mathbb{C} \backslash[0, \infty)$.

The basic problem of spectral theory is to analyze the behavior of $R(z)$ as $z$ approaches $\left[\inf _{\partial X} V, \infty\right)$. We shall only do this in any detail for the metric (1) and for $V \in x^{2} \mathcal{C}^{\infty}(X)$. The approach here is via commutator estimates; we give a good understanding of the 'strong limit'

$$
\left(\Delta+V-(\lambda \pm i 0)^{2}\right)^{-1}: \dot{\mathcal{C}}^{\infty}(X) \longrightarrow \mathcal{C}^{-\infty}(X)
$$

but leave open the possible development of a 'large calculus' which would actually contain the limiting operator. 


\section{Section 7: Wavefront set.}

The notion of wavefront set is based on a notion of operator wavefront set, or essential support. For scattering pseudodifferential operators this is described in Appendix B; in the case of $\mathbb{S}_{+}^{n}$ it amounts to the essential support of the amplitude $a_{L}$ in (4.1). More abstractly, the spaces $\Psi_{\mathrm{sc}}^{m, l}(X) / \Psi_{\mathrm{sc}}^{-\infty, \infty}(X)$ form a sheaf of algebras over $C_{\mathrm{sc}} X$ with support

$$
\mathrm{WF}_{\mathrm{sc}}^{\prime}(A) \subset C_{\mathrm{sc}} X \quad \forall A \in \Psi_{\mathrm{sk}}^{m, l}(X)
$$

The scattering pseudodifferential operators are microlocal in the sense that

$$
\mathrm{WF}_{\mathrm{sc}}^{\prime}(A \circ B) \subset \mathrm{WF}_{\mathrm{sc}}^{\prime}(A) \cap \mathrm{WF}_{\mathrm{sc}}^{\prime}(B)
$$

Moreover the closure of the elliptic set is a lower bound for the operator wavefront set. Thus if $\operatorname{ell}_{\mathrm{sc}}^{m, l}(A)$ is the set where $j_{\mathrm{sc}, m, l}(A) \neq 0$ for $A \in \Psi_{\mathrm{sc}}^{m, l}(X)$

$$
\mathrm{ell}_{\mathrm{sc}}^{m, l}(A) \subset \mathrm{WF}_{\mathrm{sc}}^{\prime}(A) \quad \forall A \in \Psi_{\mathrm{sc}}^{m, l}(X) .
$$

The complement of the elliptic set is the (scattering) characteristic set:

$$
\Sigma_{\mathrm{sc}}(P)=C_{\mathrm{sc}} X \backslash \operatorname{ell}_{\mathrm{sc}}^{m, l}(P)
$$

The quantization map can also be selected to be microlocal in this sense, so that

$$
\mathrm{WF}_{\mathrm{sc}}^{\prime}\left(q_{m, l}(a)\right)=\operatorname{esssup}(a)
$$

where the essential support of $a \in \mathcal{A}^{m, l}\left(\mathrm{sc}^{*} X\right)$ is the subset of $C_{\mathrm{sc}} X$ near which it is not equal to an element of $\dot{\mathcal{C}}^{\infty}\left({ }^{\mathrm{sc}} \bar{T}^{*} X\right)$. In fact

$$
\mathrm{WF}_{\mathrm{sc}}^{\prime}(A)=\emptyset \Longleftrightarrow A \in \Psi_{\mathrm{sc}}^{-\infty, \infty}(X)
$$

For distributions the 'absolute' scattering wavefront set is defined by

$$
\mathrm{WF}_{\mathrm{sc}}(u)=\bigcap\left\{\Sigma_{\mathrm{sc}}(A) ; A \in \Psi_{\mathrm{sc}}^{m, l}(X) \text { and } A u \in \dot{\mathcal{C}}^{\infty}(X)\right\}
$$

and then:

Proposition 6. For any $A \in \Psi_{\mathrm{scc}}^{m, l}(X)$ and any $u \in \mathcal{C}^{-\infty}(X)$

$$
\mathrm{WF}_{\mathrm{sc}}(A u) \subset \mathrm{WF}_{\mathrm{sc}}^{\prime}(A) \cap \mathrm{WF}_{\mathrm{sc}}(u)
$$


In particular a relative version of this wavefront set, corresponding to the Sobolev spaces $H_{\mathrm{sc}}^{m, l}(X)$, is also useful:

$$
\begin{aligned}
& C_{\mathrm{sc}} X \backslash \mathrm{WF}_{\mathrm{sc}}^{m, l}(u)=\left\{p \in C_{\mathrm{sc}} X ; \exists A \in \Psi_{\mathrm{sc}}^{m,-l}(X)\right. \\
&\text { with } \left.j_{\mathrm{sc}, m, l}(A)(p) \neq 0 \text { and } A u \in L_{\mathrm{sc}}^{2}(X)\right\} .
\end{aligned}
$$

This can be written as in (7.7)

$$
\mathrm{WF}_{\mathrm{sc}}^{m, l}(u)(u)=\bigcap\left\{\Sigma_{\mathrm{sc}}(A) ; A \in \Psi_{\mathrm{sc}}^{m,-l}(X) \text { and } A u \in L_{\mathrm{sc}}^{2}(X)\right\}
$$

Clearly $\mathrm{WF}_{\mathrm{sc}}(u) \supset \mathrm{WF}_{\mathrm{sc}}^{m, l}(u)$ for any $m, l$ and basic elliptic regularity shows that

$$
\begin{gathered}
\text { if } u \in \mathcal{C}^{-\infty}(X), \mathrm{WF}_{\mathrm{sc}}^{m, l}(u)=\emptyset \Longleftrightarrow u \in H_{\mathrm{sc}}^{m, l}(X) \\
\text { if } u \in \mathcal{C}^{-\infty}(X), A \in \Psi_{\mathrm{sc}}^{m,-l}(X) \text { then } A u \in H_{\mathrm{sc}}^{M, L}(X) \\
\Longrightarrow \mathrm{WF}_{\mathrm{sc}}^{M+m, L+l}(u) \cap \operatorname{ell}_{\mathrm{sc}}^{m,-l}(A)=\emptyset .
\end{gathered}
$$

In the sequel we often wish to consider convergence of functions depending on a parameter and it is therefore convenient to extend the notion of wavefront set to families. Let $B$ be a metric space and let $L^{\infty}\left(B ; H_{\mathrm{sc}}^{M, L}(X)\right)$ be the space of bounded maps $B \longrightarrow H_{\mathrm{sc}}^{M, L}(X)$. Typically we denote such a map by $u_{t}, t \in B$. Since $\mathcal{C}^{-\infty}(X)$ is an LF space

$$
L^{\infty}\left(B ; \mathcal{C}^{-\infty}(X)\right)=\bigcup_{M, L} L^{\infty}\left(B ; H_{\mathrm{sc}}^{M, L}(X)\right)
$$

Definition 4. If $u_{t} \in L^{\infty}\left(B ; \mathcal{C}^{-\infty}(X)\right)$ and $m, l \in \mathbb{R}$ then

$$
\begin{aligned}
& C_{\mathrm{sc}} X \backslash \mathrm{WF}_{\mathrm{sc}, L^{\infty}(B)}^{m, l}\left(u_{t}\right)=\left\{p \in C_{\mathrm{sc}} X ; \exists A \in \Psi_{\mathrm{sc}}^{m,-l}(X)\right. \\
& \text { with } \left.j_{\mathrm{sc}, m,-l}(p) \neq 0 \text { and } A u_{t} \in L^{\infty}\left(B ; L_{\mathrm{sc}}^{2}(X)\right)\right\} .
\end{aligned}
$$

Notice that we only define $\mathrm{WF}_{\mathrm{sc}, L^{\infty}(B)}^{m, l}$ for bounded families. Although we have taken the operator $A$ in (7.13) to be independent of $t \in B$ this is by no means necessary.

Lemma 7. Let $B \ni t \longmapsto A_{t} \in \Psi_{\mathrm{sc}}^{m,-l}(X)$ be a continuous map with all semi norms uniformly bounded for $t \in B$ and suppose that for some $\delta>0$ and some open set $G \subset C_{\mathrm{sc}} X$

$$
\left|j_{\mathrm{sc}, m,-l}\left(A_{t}\right)(p)\right| \geq \delta \quad \forall p \in G
$$


then

$$
\begin{gathered}
u_{t} \in L^{\infty}\left(B ; \mathcal{C}^{-\infty}(X)\right) \text { and } A_{t} u_{t} \in L^{\infty}\left(B ; L_{\mathrm{sc}}^{2}(X)\right) \\
\Longrightarrow \mathrm{WF}_{\mathrm{sc}, L^{\infty}(B)}^{m, l}\left(u_{t}\right) \cap G=\emptyset .
\end{gathered}
$$

\section{Section 8: Principal-type propagation.}

Using the formulæ (5.24), (5.25) and (5.16) for the symbol of a commutator an analogue, for scattering pseudodifferential operators, of Hörmander's theorem on the propagation of singularities is derived. Indeed the only cases of direct interest here concern the codimension-one boundary points of ${ }^{\mathrm{sc}} \bar{T}^{*} X$, at which the result actually reduces to the standard one using Proposition 2. Nevertheless we briefly discuss the positive-commutator proof from [3] and then use it to extend the result to a uniform one for the spectral family.

Proposition 7. Suppose $P \in \Psi_{\mathrm{sc}}^{m, l}(X)$ has real joint symbol, then for any $M, L \in \mathbb{R}$ and any $u \in \mathcal{C}^{-\infty}(X)$

$$
\mathrm{WF}_{\mathrm{sc}}^{M, L}(u) \backslash \mathrm{WF}_{\mathrm{sc}}^{M-m+1, L+l+1}(P u)
$$

is a union of maximally extended integral curves of ${ }^{\mathrm{sc}} H_{p}^{m, l}, p=j_{\mathrm{sc}, m, l}(P)$, in

$$
\Sigma_{\mathrm{sc}}(P) \backslash \mathrm{WF}_{\mathrm{sc}}^{M-m+1, L+l+1}(P u) .
$$

Proof: If $A$ and $B$ are elliptic operators with real principal symbols then the validity of the result for $P$ and $u$ follows from its validity for $A P B$ and $v=Q u$ where $Q$ is a parametrix for $B$. Thus it suffices to prove the proposition for $m=1$, $l=-1$ and $M=L=0$.

Elliptic regularity shows that the set in (8.1) is contained in the set in (8.2). As with all propagation results concerning flows, it suffices to prove the local version. Namely

$$
\begin{gathered}
\text { if } q \in \Sigma_{\mathrm{sc}}(P), q \notin \mathrm{WF}_{\mathrm{sc}}^{0,0}(P u) \text { and } \\
\text { for some } r_{0}>0 \exp \left(t^{\mathrm{sc}} H_{p}^{1,-1}\right)(q) \notin \mathrm{WF}_{\mathrm{sc}}^{0,0}(u) \text { for } r \in\left(0, r_{0}\right) \\
\text { then } q \notin \mathrm{WF}_{\mathrm{sc}}^{0,0}(u) .
\end{gathered}
$$

The alternate assumption that $\exp \left(t^{\mathrm{sc}} H_{p}^{1,-1}\right)(q) \notin \mathrm{WF}_{\mathrm{sc}}^{0,0}(u)$ for $r \in\left(-r_{0}, 0\right)$ leads to the same conclusion by reversing the sign of $P$. The implication in (8.3) is also trivially valid if ${ }^{\mathrm{sc}} H_{p}^{1,-1}$ vanishes at $q$. It is enough to prove (8.3) for $r_{0}>0$ small enough, depending on $q$.

If $q$ lies in the interior of ${ }^{\mathrm{sc}} S^{*} X$ then the local result is just the local form of Hörmander's propagation theorem. To prove the general result, especially near the 
corner of ${ }^{\mathrm{sc}} \bar{T}^{*} X$ we briefly recall the structure of the positive-commutator proof, in the context of the scattering calculus.

Let $\gamma$ be a closed neighbourhood of $q$ in $C_{\mathrm{sc}} X$ and let $r_{0}>0$ be such that the point $\exp \left(r^{\mathrm{sc}} H_{p}^{1,-1}\right)(q)$ lies in the interior of $\gamma$ for $r \in\left[0, r_{0}\right]$; let $\gamma^{\prime}$ be a closed neighbourhood of $\exp \left(r_{0}{ }^{\mathrm{sc}} H_{p}^{1,-1}\right)(q)$. Then for any $\mu, \lambda \in \mathbb{R}$ there exists a bounded family $B_{t} \in L^{\infty}\left((0,1) ; \Psi_{\mathrm{scc}}^{\mu, \lambda}(X)\right)$, with various properties. First concerning its boundedness and regularity

$$
\mathrm{WF}_{\mathrm{sc}, L^{\infty}((0,1))}^{\prime}\left(B_{t}\right) \subset \gamma, B_{t}^{*}=B_{t}
$$

and

$$
\begin{gathered}
{[0,1] \ni t \longmapsto B_{t} \in \Psi_{\mathrm{sc}}^{\mu+\delta, \lambda-\delta}(X) \text { is continuous for } \delta>0 \text { with }} \\
B_{0} \in \Psi_{\mathrm{sc}}^{\mu, \lambda}(X) \text { and } B_{t} \in \Psi_{\mathrm{sc}}^{-\infty, \infty}(X) \text { for } t>0 .
\end{gathered}
$$

The main properties are for the 'commutator' with the square:

$$
\frac{1}{2 i}\left(B_{t}^{2} P-P^{*} B_{t}^{2}\right)=G_{t}^{2}+E_{t}+F_{t}
$$

where all three families on the right are of order $-\infty, \infty$ for $t>0$ and satisfy

$$
\begin{gathered}
\mathrm{WF}_{\mathrm{sc}, L^{\infty}(B)}^{\prime}\left(E_{t}\right) \subset \gamma^{\prime}, E_{t} \in L^{\infty}\left(X ; \Psi_{\mathrm{scc}}^{2 \mu, 2 \lambda} B\right) \\
\mathrm{WF}_{\mathrm{sc}, L^{\infty}(B)}^{\prime}\left(F_{t}\right) \subset \gamma, F_{t} \in L^{\infty}\left(X ; \Psi_{\mathrm{scc}}^{2 \mu, 2 \lambda} B\right) \\
\mathrm{WF}_{\mathrm{sc}, L^{\infty}(B)}^{\prime}\left(G_{t}\right) \subset \gamma, G_{t} \in L^{\infty}\left(X ; \Psi_{\mathrm{scc}}^{\mu, \lambda} B\right) \\
\text { with }[0,1] \ni t \longmapsto G_{t} \in \Psi_{\mathrm{sc}}^{\mu+\delta, \lambda-\delta}(X) \text { continuous for } \delta>0, \\
G_{t}^{*}=G_{t}, G_{0} \in \Psi_{\mathrm{sc}}^{\mu, \lambda}(X) \text { and } q \in \operatorname{ell}_{\mathrm{sc}}^{\mu, \lambda}\left(G_{0}\right) .
\end{gathered}
$$

Assuming that such families have been constructed it is then easy to prove the local result by iteration. In $t>0$ the inequality

$$
\operatorname{Im}\left\langle u, B_{t}^{2} P u\right\rangle \leq\left|\left\langle u, B_{t}^{2} P u\right\rangle\right|
$$

can be written as a bound on the $L^{2}$ norm:

$$
\left\|G_{t} u\right\|^{2} \leq\left|\left\langle u, E_{t} u\right\rangle\right|+\left|\left\langle u, F_{t} u\right\rangle\right|+\left|\left\langle u, B_{t}^{2} P u\right\rangle\right| .
$$

Under the assumptions of (8.3) set $S=\sup \left\{s ; q \notin \mathrm{WF}_{\mathrm{sc}}^{s,-s}(u)\right\}$. Thus the local statement in (8.3) is just that $S \geq 0$ and that if $S=0$ then $q \notin \mathrm{WF}_{\mathrm{sc}}^{0,0}(u)$. Certainly $S$ is finite and the result is trivially true if $S>0$. Thus, supposing that $S \leq 0$, choose $(\mu, \lambda)=(s,-s)$ where $S \leq s, s<S+\frac{1}{2}, s \leq 0$ and $s>S$ unless $S=0$. As $t \downarrow 0$ all the terms on the right in (8.8) remain bounded. Thus $G_{t} u$ is bounded 
in $L^{2}$. Since $G_{t} u \rightarrow G_{0} u$ in distributions it follows that $G_{0} u \in L^{2}$. Thus $S<0$ is not possible, since then $q \notin \mathrm{WF}_{\mathrm{sc}}^{s,-s}(u)$ with $s>S$ and if $S=0$ it follows that $q \notin \mathrm{WF}_{\mathrm{sc}}^{0,0}(u)$ as expected.

Thus the result follows once such families, $B_{t}$, satisfying (8.4), (8.5) and (8.7) have been constructed. In fact this just reduces to the solution of the transport equation. Suppose that $B_{t}$ is given by a quantization map, $B_{t}=q\left(b_{t}\right)$. Then it follows that

$$
B_{t}^{2} P-P^{*} B_{t}^{2}-\frac{1}{i} q\left({ }^{\mathrm{sc}} H_{p}^{1,-1} b_{t}^{2}-a b_{t}^{2}\right)=F_{t},
$$

where $a$ is the principal symbol of $i\left(P-P^{*}\right)$, is as required in (8.7). It remains then to construct $b_{t}$ with appropriate order properties such that

$$
{ }^{\mathrm{sc}} H_{p}^{1,-1} b_{t}^{2}-a b_{t}^{2}=g_{t}^{2}+e_{t} .
$$

where $e_{t}$ is to have support in $\gamma^{\prime}$ and by assumption ${ }^{\mathrm{sc}} H_{p}^{1,-1} \neq 0$ at $q$. Since ${ }^{\mathrm{sc}} H_{p}^{1,-1}$ is a smooth, real vector field non-vanishing at $q$ and tangent to the boundary of the manifold with corners ${ }^{\mathrm{sc}} \bar{T}^{*} X$ local coordinates $x_{i}, y_{j}$ can be introduced where $x_{i}=0$ are the boundary hypersurfaces locally near $q$ (there are either two or one of these as $q$ lies in the corner of not) and ${ }^{\mathrm{sc}} H_{p}^{1,-1}=\partial / \partial y_{1}$. The symbolic conditions on $b^{t}$ amount to conormal estimates; an explicit solution in these coordinates is given by

$$
b_{t}=\left(1-\phi\left(t x_{1}\right)\right)\left(1-\phi\left(t x_{1}\right)\right) x_{1}^{\mu} x_{2}^{\lambda} \exp \left(\frac{1}{2} A y_{1}\right) \psi\left(x, y^{\prime}\right) \exp \left(-\frac{1}{2\left(y_{1}-s\right)^{2}}\right) g\left(y_{1}\right)
$$

in $y_{1} \geq s$ and $b_{t}=0$ in $y_{1}<s$. Here $s<0$ is chosen small, $\psi$ is a smooth function of small compact support in the remaining variables $x$ and $y_{j}$ for $j \geq 2$, with $\psi(0)=1, A>0$ is a constant to be chosen large enough (compared to $a$ ), $\phi \in \mathcal{C}_{c}^{\infty}(\mathbb{R})$ is non-negative and is identically equal to 1 near 0 and $g \in \mathcal{C}_{c}^{\infty}(\mathbb{R})$ is non-negative, identically equal to 1 in $s<y_{1}<r+s$, and identically 0 in $y_{1}>r_{0}-s$.

Notice that in this proof no use is made of the fact that the test operator, $B_{t}^{2}$, is a square. Reversing the direction of ${ }^{\mathrm{sc}} H_{p}^{1,-1}$ amounts to reversing the sign of $P$ and this in turn reverses the relative signs of $b_{t}^{2}$ and $g_{t}^{2}$ in (8.10). This has an immediate effect when we consider operators for which the principal symbol has an imaginary part of fixed sign, see [3]. We are only interested here in the particularly simple case of the resolvent family as the spectral parameter approaches the real axis.

Proposition 8. Suppose $P \in \Psi_{\mathrm{sc}}^{m, l}(X)$ has real joint symbol, $G \subset(0, \infty)$ is bounded and that $u_{\tau} \in L^{\infty}\left(G ; \mathcal{C}^{-\infty}(X)\right)$ then for any $M, L \in \mathbb{R}$

$$
\mathrm{WF}_{\mathrm{sc}, L^{\infty}(G)}^{M, L}(u) \backslash \mathrm{WF}_{\mathrm{sc}, L^{\infty}(G)}^{M-m+1, L+l+1}\left((P-i \tau) u_{\tau}\right)
$$


is a union of maximally forward-extended integral curves of ${ }^{\mathrm{sc}} H_{p}^{m, l}, p=j_{\mathrm{sc}, m, l}(P)$ in

$$
\Sigma_{\mathrm{sc}}(P) \backslash \mathrm{WF}_{\mathrm{sc}, L^{\infty}(G)}^{M-m+1, L+l+1}\left((P-i \tau) u_{\tau}\right) .
$$

Of course this result applies equally well when $G$ is a bounded subset of $(-\infty, 0)$ by reversing the sign of $P$.

ProOF: We shall only prove this result under the additional assumption that $m \leq 0$ and $l \geq 0$. Then $i \tau$ is at least as important as the principal symbol. In the other case, away from the corner of ${ }^{\mathrm{sc}} \bar{T}^{*} X$, the result essentially reduces to Proposition 7 . This assumption on the order means that the family $P-i \tau$ is elliptic when $\tau \neq 0$, so there is no need to regularize the solution.

Only minor modifications are needed to the proof of Proposition 7. In particular the symbol (8.11) can be used with $t=0$. The identity (8.9) is therefore replaced by

$$
B_{t}^{2}(P-i \tau)-\left(P^{*}+i \tau\right) B_{t}^{2}-\frac{1}{i}\left(2 \tau B_{t}^{2}+q\left(\rho_{\sigma}^{m-1} \rho_{\partial}^{l+1 \mathrm{sc}} H_{p}^{m, l} b_{t}^{2}-a b_{t}^{2}\right)\right)=F_{t}
$$

This just leads to

$$
\frac{1}{2 i}\left(B_{t}^{2} P-P^{*} B_{t}^{2}\right)=\tau B_{t}^{2}+G_{t}^{2}+E_{t}+F_{t}
$$

as a replacement for (8.6). The extra term, $\tau B_{t}^{2}$, appears on the left in (8.8) and so can be dropped, as $\tau \geq 0$. Thus the same estimate follows, uniformly in $\tau \in G$.

Applying this result to the resolvent family for the Laplacian gives useful information at points on the characteristic variety where the Hamilton vector field is non-vanishing. For the operator $\Delta-\lambda^{2}, \lambda \neq 0$, the characteristic variety is the surface

$$
\Sigma_{\mathrm{sc}}\left(\Delta-\lambda^{2}\right)=\left\{|\zeta|^{2}=\lambda^{2}\right\} .
$$

From (5.24) the Hamilton vector field can be written, near $\Sigma_{\mathrm{sc}}\left(\Delta-\lambda^{2}\right)$,

$$
{ }^{\mathrm{sc}} H_{|\zeta|^{2}-\lambda^{2}}^{2,0}=2 \tau\left(x \frac{\partial}{\partial x}+R_{\mu}\right)-2|\mu|^{2} \frac{\partial}{\partial \tau}+H_{h}+x V, V \in \mathcal{V}_{\mathrm{b}}\left({ }^{\mathrm{sc}} T^{*} X\right),
$$

where $H_{h}$ is the Hamilton vector field of the metric function on $T^{*} \partial X$. Here we are using the natural choice of defining function, $x$, associated to the metric to decompose the inward-pointing normal bundle to ${ }^{\mathrm{sc}} T_{\partial X}^{*} X$, as a boundary hypersurface of ${ }^{\mathrm{sc}} T^{*} X$, as

$$
N^{+}\left({ }^{\mathrm{sc}} T_{\partial X}^{*} X\right)=[0, \infty)_{x} \times \mathbb{R}_{\tau} \times T^{*} \partial X .
$$




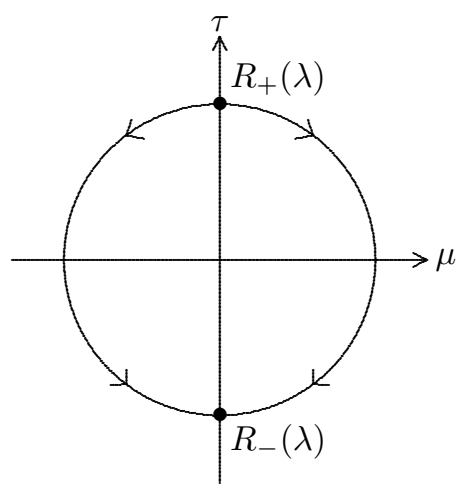

Figure 2. Principal-type propagation for $\Delta-(\lambda+i 0)^{2}, \lambda>0$..

We have also simply dropped the factor $|\zeta|^{m-1}$ since this is non-zero near the interior of ${ }^{\mathrm{sc}} T_{\partial X}^{*} X$.

We are therefore interested in the structure of the null integral curves of ${ }^{\mathrm{sc}} H_{|\zeta|^{2}-\lambda^{2}}^{2,0}$, i.e. those in $\Sigma_{\mathrm{sc}}\left(\Delta-\lambda^{2}\right)$, which itself lies inside $x=0$. Thus

$$
{ }^{\mathrm{sc}} H_{|\zeta|^{2}-\lambda^{2}}^{2,0}=2 \tau R_{\mu}-2|\mu|^{2} \frac{\partial}{\partial \tau}+H_{h} \text { on } \Sigma_{\mathrm{sc}}\left(\Delta-\lambda^{2}\right) .
$$

The tangent term $H_{h}$ commutes with the whole of the vector field so it is natural to consider the 'normal' part $2 \tau R_{\mu}-2|\mu|^{2} \partial / \partial \tau$. This vanishes precisely at $\mu=0$ so the three subsets of the characteristic variety

$$
\begin{gathered}
R_{ \pm}(\lambda)=\{\mu=0, \tau= \pm \lambda\} \subset{ }^{\mathrm{sc}} \bar{T}_{\partial X}^{*} X \\
\Sigma_{\mathrm{sc}}\left(\Delta-\lambda^{2}\right) \backslash\left(R_{+}(\lambda) \cup R_{-}(\lambda)\right) .
\end{gathered}
$$

are each invariant under the bicharacteristic flow.

In $R_{ \pm}(\lambda)$ the integral curves are just geodesics in $\partial X$. The remaining, open, part of the characteristic variety is fibred by integral curves, $\exp \left(r^{\mathrm{sc}} H_{|\zeta|^{2}-\lambda^{2}}^{2,0}\right)$, which each approach $R_{-}(\lambda)$ uniformly as $r \rightarrow(\operatorname{sgn} \lambda) \infty$ and $R_{+}(\lambda)$ as $r \rightarrow-(\operatorname{sgn} \lambda) \infty$. Thus the results given above cannot be used to determine whether $R_{ \pm}(\lambda)$ as a whole is in the wavefront set of a solution, but can be used to analyse the propagation of singularities (or regularity) from $R_{ \pm}(\lambda)$ into the set (8.21). We therefore think of $R_{ \pm}(\lambda)$ as 'radial' surfaces for the operator $\Delta-\lambda^{2}$, although they might better be thought of as 'radially radial' surfaces!

This is illustrated in Figure 2 and discussed further in $\S 14$ below. 


\section{Section 9: Radial surfaces.}

In this section we prove results concerning the propagation of singularities for $\Delta-(\lambda+i t)^{2}$ near the radial surfaces (8.20). This is somewhat more complicated than the principal-type propagation discussed above. In particular there is a fundamental distinction for regularity with respect to the scattering Sobolev spaces $H_{\mathrm{sc}}^{M, s}(X)$ between the cases $s \geq-\frac{1}{2}$ and $s<-\frac{1}{2}$, which reflects the existence of asymptotic solutions as in (4). In each of these two cases we prove two results, one uniform in $t$ (and depending on the sign) the other only for the case $t=0$.

Since all four propagation results depend on the construction of appropriate test operators we start with a general result which covers all cases.

Lemma 8. If $B_{r} \in L^{\infty}\left(B ; \Psi_{\mathrm{scc}}^{-\infty, S}(X)\right)$ is self-adjoint with respect to the metric volume form and has symbol $b_{r}=b_{r}(x, \tau,|\mu|)$ in terms of a decomposition (2.1) then

$$
\left(\Delta-(\lambda+i t)^{2}\right) B_{r}^{2}-B_{r}^{2}\left(\Delta-(\lambda-i t)^{2}\right)=-4 i t \lambda B_{r}^{2}+2 i x C_{r, t}
$$

where $C_{r} \in L^{\infty}\left(B \times G ; \Psi_{\mathrm{scc}}^{-\infty, 2 S}(X)\right)$ for any bounded $G \subset \mathbb{R}$ has symbol

$$
c_{r}=-|\mu|^{2} \frac{\partial}{\partial \tau} b_{r}^{2}+\tau R_{\mu} b_{r}^{2}+2 \tau x \frac{\partial}{\partial x} b_{r}^{2}
$$

Proof: This follows directly from (5.23).

By making appropriate choices of $b$ so that the leading part of $C_{r}$ is a sum of squares (to avoid use of Gårding-type inequalities) we can proved propagation results which are global near $R_{ \pm}(\lambda)$. In all cases the 'interior' regularity order is totally irrelevant and is therefore written $*$. First we give conditions under which $R_{ \pm}(\lambda)$ cannot be an isolated subset of the wavefront set.

Proposition 9. If $\lambda \neq 0, s<-\frac{1}{2}$ and $u \in \mathcal{C}^{-\infty}(X)$ then

$$
\begin{gathered}
R_{ \pm}(\lambda) \cap \mathrm{WF}_{\mathrm{sc}}^{*, s+1}\left(\left(\Delta-\lambda^{2}\right) u\right)=\emptyset \text { and } R_{ \pm}(\lambda) \cap \operatorname{cl}\left(\mathrm{WF}_{\mathrm{sc}}^{*, s}(u) \backslash R_{ \pm}(\lambda)\right)=\emptyset \\
\Longrightarrow \mathrm{WF}_{\mathrm{sc}}^{*, s}(u) \cap R_{ \pm}(\lambda)=\emptyset .
\end{gathered}
$$

If $G \subset(0, \infty)$ or $G \subset(-\infty, 0)$ is bounded and $u_{t} \in L^{\infty}\left(G ; \mathcal{C}^{-\infty}(X)\right)$ then

$$
\begin{gathered}
R_{-\operatorname{sgn} G}(\lambda) \cap \mathrm{WF}_{\mathrm{sc}, L^{\infty}(G)}^{*, s+1}\left(\left(\Delta-(\lambda+i t)^{2}\right) u_{t}\right)=\emptyset \text { and } \\
R_{-\operatorname{sgn} G}(\lambda) \cap \operatorname{cl}\left(\mathrm{WF}_{\mathrm{sc}, L^{\infty}(G)}^{*,}\left(u_{t}\right) \backslash R_{-\operatorname{sgn} G}(\lambda)\right)=\emptyset \\
\Longrightarrow \mathrm{WF}_{\mathrm{sc}, L^{\infty}(G)}^{*}\left(u_{t}\right) \cap R_{-\operatorname{sgn} G}(\lambda)=\emptyset .
\end{gathered}
$$

Proof: To prove (9.3) we shall apply Lemma 8 with $B=[0,1]$ and with the symbol of $B_{r}$ taken in the form

$$
b_{r}=b \phi_{3}\left(\frac{x}{r}\right), b=x^{-s-\frac{1}{2}} \phi_{1}\left(\frac{\tau^{2}+|\mu|^{2}}{\lambda^{2}}\right) \phi_{2}\left( \pm \frac{\tau}{|\mu|}\right) \phi_{4}(x) .
$$


Given $\delta>0$ we shall take $\phi_{1}(z) \in \mathcal{C}^{\infty}(\mathbb{R})$ to have support in $\lambda^{2}-\delta<z<\lambda^{2}+\delta$ and to be positive definite in the interior of this set. We shall take $\phi_{2} \in \mathcal{C}^{\infty}(\mathbb{R})$ to have support in $[1 / \delta, \infty)$, to be positive in the interior and to be identically equal to 1 in $[2 / \delta, \infty), \phi_{3} \in \mathcal{C}^{\infty}(\mathbb{R})$ to vanish in $(-\infty, 1)$ and to be identically equal to 1 in $(2, \infty)$ and finally $\phi_{4} \in \mathcal{C}^{\infty}(\mathbb{R})$ should be identically equal to 1 near 0 and have support in the product neighbourhood of the boundary. Thus $b_{r}$ is well-defined and bounded in $\mathcal{A}^{\left(-\infty,-s-\frac{1}{2}\right)}\left({ }^{\mathrm{sc}} \bar{T}^{*} X\right)$ for $r \in[0,1]$.

We shall choose $\phi_{2}$ and $\phi_{3}$ so that $c_{r}$, given by (9.2), is a sum of squares of symbols. The factor involving $\phi_{1}$ is annihilated by the vector field in (9.2) so can be ignored. Similarly the factor $\phi_{4}$ is 1 near the boundary, so does not affect the symbol to any order. Thus

$$
\begin{gathered}
\pm c_{r}=|\tau|\left(2(2 s-1) x b_{r}^{2}-2 x b \phi_{3}^{\prime} \phi_{3}\left(\frac{x}{r}\right)\right)+e_{r} \\
e_{r}=e \phi_{3}^{2}\left(\frac{x}{r}\right), e=\mp 2 x^{-2 s} \phi_{1}^{2}\left(\frac{\tau^{2}+|\mu|^{2}}{\lambda^{2}}\right) \frac{\tau}{|\mu|} \phi_{2}^{\prime}\left( \pm \frac{\tau}{|\mu|}\right) \phi_{2}\left( \pm \frac{\tau}{|\mu|}\right) \phi_{4}(x) .
\end{gathered}
$$

For an appropriate choice of $\phi_{3}$ the first two terms are squares of symbols; the third term has support disjoint from $R_{ \pm}(\lambda)$. Thus

$$
c_{r}=a^{2} \phi_{3}^{2}\left(\frac{x}{r}\right)+f_{r}^{2}+g_{r},
$$

where $a=(2|\tau|(-2 s-1))^{\frac{1}{2}} x^{\frac{1}{2}} b$ is a symbol of order $-s$, elliptic in a neighbourhood of $R_{ \pm}(\lambda), f_{r}$ is bounded in $\mathcal{A}^{(-\infty,-s)}\left({ }^{\mathrm{sc}} \bar{T}^{*} X\right)$ and $g_{r}$ is bounded in $\mathcal{A}^{(-\infty,-2 s)}\left(\mathrm{sc} \bar{T}^{*} X\right)$ and has support disjoint from $R_{ \pm}(\lambda)$. Each term has essential support in a neighbourhood of $R_{ \pm}(\lambda)$ which becomes small with $\delta$.

Now consider the identity (9.1) for $t=0$, which can be written

$$
\pm\left(\left(\Delta-\lambda^{2}\right) B_{r}^{2}-B_{r}^{2}\left(\Delta-\lambda^{2}\right)\right)=2 i\left(A_{r}^{2}+F_{r}^{2}+G_{r}\right)
$$

where $A_{r}$ and $F_{r}$ are self-adjoint with symbols $a \phi_{3}\left(\frac{x}{r}\right)$ and $f_{r}$ respectively and $G_{r}$ is bounded of order $-s$ with essential support disjoint from $R_{ \pm}(\lambda)$. Since all terms in (9.4) are of order $\infty$ for $r>0$ pairing with $u$ is permissible and gives

$$
\mp \operatorname{Im}\left\langle B_{r}^{2} u,\left(\Delta-\lambda^{2}\right) u\right\rangle=\left\|A_{r} u\right\|^{2}+\left\|F_{r} u\right\|^{2}+\left\langle G_{r} u, u\right\rangle .
$$

Using the Cauchy-Schwarz inequality

$$
\left|\left\langle B_{r}^{2} u,\left(\Delta-\lambda^{2}\right) u\right\rangle\right| \leq \epsilon^{2}\left\|A_{r} u\right\|^{2}+C \epsilon^{-2}\left\|x^{-\frac{1}{2}} B_{r}\left(\Delta-\lambda^{2}\right) u\right\|^{2}, \epsilon>0 .
$$

Since the symbol of $A_{r}$ is an elliptic multiple of the symbol of $x^{\frac{1}{2}} B_{r}$ near $R_{ \pm}(\lambda)$, (9.7) leads to a uniform estimate

$$
\left\|x^{\frac{1}{2}} B_{r} u\right\|^{2} \leq C\left(\left\|x^{-\frac{1}{2}} B_{r}\left(\Delta-\lambda^{2}\right) u\right\|^{2}+\left\|E_{r} u\right\|^{2}+\left\|H_{r} u\right\|^{2}\right)
$$


where $E_{r} \in L^{\infty}\left(B ; \Psi_{\mathrm{scc}}^{-s+1}(X)\right)$ and $H_{r} \in L^{\infty}\left(B ; \Psi_{\mathrm{scc}}^{-s}(X)\right)$ have essential support near $R_{ \pm}(\lambda)$ and that of $H_{r}$ is disjoint from $R_{ \pm}(\lambda)$.

Applying this estimate iteratively in $s$ we may suppose that $E_{r}$ has any given order. This gives the estimate (9.3) on the wavefront set since the assumption in (9.3) implies that all terms on the right in (9.9) are bounded. The ellipticity of $B_{0}$ on $R_{ \pm}(\lambda)$ then implies that $\mathrm{WF}_{\mathrm{sc}}^{(*, s)}(u)$ does not meet $R_{ \pm}(\lambda)$.

The proof of $(9.4)$ is very similar. Let us take $G \subset(0, \infty)$ for the sake of definiteness. Under the hypotheses of $(9.4), \mathrm{WF}_{\mathrm{sc}, L^{\infty}(G)}^{*, s+1}\left(u_{t}\right)$ does not meet $R_{-}(\lambda)$ for $t \in G$. Thus it is not necessary to use the family $B_{r}$ since integration by parts involving $B=B_{0}$ is already justified. Taking into account that $t \neq 0,(9.8)$ is replaced by

$$
-\operatorname{Im}\left\langle B^{2} u,\left(\Delta-(\lambda+i t)^{2}\right) u\right\rangle=-2 \lambda t\|B u\|^{2}+\|A u\|^{2}+\|F u\|^{2}+\langle G u, u\rangle .
$$

Since the symbols are supported near $\tau=-\lambda \operatorname{sgn} G,-\lambda t \geq 0$, and the argument proceeds as before, giving (9.4).

When we pass to the consideration the finer regularity corresponding to $s \geq \frac{1}{2}$ three related changes occur. First the sign of the main term in the commutator is reversed relative to the sign of the commutant. This means that the cutoff factor $\phi_{3}$ in (9.5) must be changed but on the other hand means the term coming from the cutoff $\phi_{2}$ is now positive. This allows us to obtain the following 'absolute' regularity result.

Proposition 10. If $\lambda \neq 0, s \geq-\frac{1}{2}$ and $u \in \mathcal{C}^{-\infty}(X)$ then

$$
\begin{gathered}
R_{ \pm}(\lambda) \cap \mathrm{WF}_{\mathrm{sc}}^{*, s+1}\left(\left(\Delta-\lambda^{2}\right) u\right)=\emptyset \text { and } R_{ \pm}(\lambda) \cap \mathrm{WF}_{\mathrm{sc}}^{*,-\frac{1}{2}}(u)=\emptyset \\
\Longrightarrow \mathrm{WF}_{\mathrm{sc}}^{*, s}(u) \cap R_{ \pm}(\lambda)=\emptyset .
\end{gathered}
$$

If $G \subset(0, \infty)$ or $G \subset(-\infty, 0)$ is bounded and $u_{t} \in L^{\infty}\left(B ; \mathcal{C}^{-\infty}((X))\right)$ then

$$
\begin{gathered}
\mathrm{WF}_{\mathrm{sc}, L^{\infty}(G)}^{*, s+1}\left(\left(\Delta-(\lambda+i t)^{2}\right) u_{t}\right) \cap R_{\mathrm{sgn} G}(\lambda)=\emptyset \Longrightarrow \\
\mathrm{WF}_{\mathrm{sc}, L^{\infty}(G)}^{*, s}\left(u_{t}\right) \cap R_{\mathrm{sgn} G}(\lambda)=\emptyset .
\end{gathered}
$$

Proof: To prove (9.11) we shall modify the argument of Proposition 9 by replacing the cutoff in $x$ near $x=r$ in (9.5) by

$$
\Phi_{3}(x, r)=\phi_{3}\left(\frac{x}{r}\right)+\frac{r^{-s-\frac{1}{2}}}{x^{-s-\frac{1}{2}}}\left(1-\phi_{3}\left(\frac{x}{r}\right)\right),
$$

with $\phi_{3}$ as before, so that now

$$
b_{r}=b \Phi_{3}(x, r), b=x^{-s-\frac{1}{2}} \phi_{1}\left(\frac{\tau^{2}+|\mu|^{2}}{\lambda^{2}}\right) \phi_{2}\left( \pm \frac{\tau}{|\mu|}\right) \phi_{4}(x) .
$$


For any $r>0$ the function $\Phi_{3}(x, r)$ is smooth down to $x=0$ and satisfies uniform estimates

$$
\left|\left(x \frac{\partial}{\partial x}\right)^{k} \Phi(x, r)\right| \leq C_{k} x^{-s-\frac{1}{2}} \text { in } x \leq 1 \forall k .
$$

Thus $b_{r}$ is, for $r>0$, a symbol or order 0 and is bounded as $r \downarrow 0$ as a symbol of (negative) order $-s-\frac{1}{2}$. Moreover

$$
x \frac{\partial}{\partial x}\left(x^{-s-\frac{1}{2}} \Phi_{3}(x, r)\right)=\left(-s-\frac{1}{2}\right) x^{-s-\frac{1}{2}} \phi_{3}\left(\frac{x}{r}\right)-\left(r^{-s-\frac{1}{2}}-x^{-s-\frac{1}{2}}\right) \frac{x}{r} \phi_{3}^{\prime}\left(\frac{x}{r}\right)
$$

with both terms non-positive. The identity (9.1), with $t \equiv 0$, gives the symbol (9.2) which becomes

$$
\begin{gathered}
\mp c_{r}=a_{r, 1}^{2}+a_{r, 2}^{2}+a_{r, 3}^{2} \text { where } \\
a_{r, 1}^{2}=2(2 s-1) x|\tau| b_{r}^{2} \\
a_{r, 2}^{2}=|\tau| 2 x^{-s+\frac{1}{2}} b^{2}\left(r^{-s-\frac{1}{2}}-x^{-s-\frac{1}{2}}\right) \frac{x}{r} \phi_{3}^{\prime} \phi_{3}\left(\frac{x}{r}\right) \text { and } \\
a_{r, 3}^{2}=2 x^{-2 s} \phi_{1}^{2}\left(\frac{\tau^{2}+|\mu|^{2}}{\lambda^{2}}\right)\left(\frac{\tau^{2}+|\mu|^{2}}{|\mu|}\right) \phi_{2}^{\prime}\left( \pm \frac{\tau}{|\mu|}\right) \phi_{2}\left( \pm \frac{\tau}{|\mu|}\right) \phi_{4}^{2}(x) .
\end{gathered}
$$

Note that $\phi_{2}$, which has support in $[1 / \delta, \infty)$, can be chosen so that $\phi_{2} \phi_{2}^{\prime}$ is the square of a smooth function and similarly $\phi_{3}$ can be chosen so that $\phi_{3}^{\prime} \phi_{3}$ is the square of a smooth function. Thus all three symbols $a_{r, i}, i=1,2,3$, are uniformly of order $-s$ and are of order 0 for $r>0$. Moreover $a_{r, 1}$ is of the form $f x^{\frac{1}{2}} b_{r}$ with $f>0$. The identity (9.7) is therefore replaced by

$$
\pm \operatorname{Im}\left\langle B_{r}^{2} u,\left(\Delta-\lambda^{2}\right) u\right\rangle=\left\|A_{r, 1} u\right\|^{2}+\left\|A_{r, 2} u\right\|^{2}+\left\|A_{r, 3} u\right\|^{2}+\left\langle G_{r} u, u\right\rangle
$$

with $G_{r}$ uniformly of multiorder $-\infty, 2 s+1$. Using the Cauchy-Schwarz inequality as in (9.8) and iterating in $s$ therefore leads to (9.11).

In the proof of (9.12) the multiplier, $B=B_{0}$, can be used directly, since no approximation is needed. The assumption that $u$ is microlocally in $x^{-\frac{1}{2}} L_{\mathrm{sc}}^{2}(X)$ on the essential support of $b_{0}$ is then not needed. The extra term $\pm 2 \lambda t\|B u\|^{2}$ appears on the right in (9.18), as in (9.10), but this can be dropped if $\pm \operatorname{sgn} \lambda t \geq 0$, i.e. $b_{0}$ has support near $R_{\mathrm{sgn} G}$, leading to (9.12).

REMARK 1. These results have been proved only for the Laplacian itself. However the proofs only depend on the properties of the normal symbol. Thus if $V$ is conormal of any positive order:

$$
\operatorname{Diff}_{\mathrm{b}}^{*}(X) V \subset x^{a} L^{\infty}(X), a>0
$$

(which includes 'long-range' potentials) the same results hold for $\Delta+V$. 


\section{Section 10: Absence of embedded eigenvalues.}

The microlocal absolute regularity in (9.11) is a starting point for a proof of global regularity. This reduces the proof of the absence of embedded eigenvalues to a unique continuation result proved by Hörmander [6].

Proposition 11. If $0 \neq \lambda \in \mathbb{R}$ and $u \in \mathcal{C}^{-\infty}(X)$ satisfies

$$
\begin{gathered}
\mathrm{WF}_{\mathrm{sc}}^{*,-\frac{1}{2}}(u) \cap\left(R_{+}(\lambda) \cup R_{-}(\lambda)\right)=\emptyset \text { and } \\
\left(\Delta-\lambda^{2}\right) u \in x^{s} H_{\mathrm{sc}}^{M}(X) \text { for some } s \geq \frac{1}{2}
\end{gathered}
$$

then $u \in x^{s-1} H_{\mathrm{sc}}^{M+2}(X)$.

Proof: In view of (10.1) and (10.2) the hypotheses of (9.11) are satisfied for both signs. Thus Proposition 10 implies that

$$
\mathrm{WF}_{\mathrm{sc}}^{*, s-1}(u) \cap\left(R_{+}(\lambda) \cup R_{-}(\lambda)\right)=\emptyset .
$$

The principal-type propagation result, Proposition 7, then shows that

$$
\mathrm{WF}_{\mathrm{sc}}^{*, s-1} \cap \Sigma_{\mathrm{sc}}(\lambda)=\emptyset .
$$

Elliptic regularity shows that $\mathrm{WF}_{\mathrm{sc}}^{M+2, s+1} \subset \Sigma_{\mathrm{sc}}(\lambda)$ and together these estimates mean that $u \in x^{s-1} H_{\mathrm{sc}}^{M+2}(X)$.

Thus as a corollary to this regularity result and [6;Theorem 17.2.8] we conclude that there are never $L^{2}$ eigenfunctions corresponding to eigenvalues embedded in the continuous spectrum.

Theorem 2. If $0 \neq \lambda \in \mathbb{R}$ and $u \in \mathcal{C}^{-\infty}(X)$ satisfies $\left(\Delta-\lambda^{2}\right) u=0$ and (10.1) then $u \equiv 0$.

Proof: Proposition 11 shows that $\mathrm{WF}_{\mathrm{sc}}(u)=\emptyset$, i.e. $u \in \dot{\mathcal{C}}^{\infty}(X)$. Now the result of Hörmander, [6;Theorem 17.2.8] applies directly in case $\partial X=\mathbb{S}^{n-1}$ with the induced metric $h$ being the standard metric on the sphere. In fact the proof given in [6] makes no use of any special properties of this metric, or the sphere itself, and applies verbatim to the present situation. Thus $u \equiv 0$.

Following Remark 1 this same result for $\Delta+V$, where $V$ satisfies (9.19) with $a \geq 1$, also follows from [6;Theorem 17.2.8].

In the basic case of the Laplacian on functions we actually conclude:

Theorem 3. If $u \in L^{2}(X)$ and $\left(\Delta-\lambda^{2}\right) u=0$ for some $\lambda \in \mathbb{C}$ then $u \equiv 0$. 
Proof: The case $\lambda \notin \mathbb{R}$ follows from the self-adjoitness of $\Delta$, i.e. directly from the existence of the resolvent family as discussed in $\S 6$. The case $0 \neq \lambda \in \mathbb{R}$ follows from Theorem 2 , since $L^{2}(X) \subset x^{-\frac{1}{2}} H_{\mathrm{sc}}^{0}(X)$. So it only remains to show that the null space of $\Delta$, acting on $L^{2}(X)$ is empty. This follows by integration by parts. Since an element of the null space satisfies the equation $(x B x) u=0$, with $B \in \operatorname{Diff}_{\mathrm{b}}^{2}(X)$ elliptic, it follows (see [7]) that $u$ is an element of $x^{\epsilon} H_{\mathrm{b}}^{\infty}(X)$ for $\epsilon>0$ sufficiently small. This, or a more direct argument, allows the integration by parts needed to prove the identity

$$
\langle\Delta u, u\rangle=\|d u\|^{2}
$$

to be justified. Thus $u$ is constant and hence, being square-integarble, vanishes identically.

The last part of this proof can be used to analyse the $L^{2}$ cohomology of a scattering metric, we state the result without proof:

Theorem 4. For a scattering metric (1) the $L^{2}$ cohomology (null space of the Laplacian on square integrable forms) is naturally isomorphic to the relative cohomology space $H^{k}(X, \partial X)$ for $k<\frac{1}{2} \operatorname{dim} X$, to the absolute cohomology $H^{k}(X)$ for $k>\frac{1}{2} \operatorname{dim} X$ and to the image of the relative in the absolute for $k=\frac{1}{2} \operatorname{dim} X$.

\section{Section 11: Sommerfeld radiation condition.}

Proposition 10, in particular (9.11), shows that the one-sided estimate on the wavefront set of $u \in \mathcal{C}^{-\infty}(X)$ :

$$
\mathrm{WF}_{\mathrm{sc}}^{*,-\frac{1}{2}}(u) \cap R_{+}(\lambda)=\emptyset
$$

has significant consequences for the global regularity of $u$. We shall call (11.1) the 'weak Sommerfeld radiation condition' and say that a distribution which satifies it is $\lambda$-outgoing.

Lemma 9. If $0 \neq \lambda \in \mathbb{R}, u \in \mathcal{C}^{-\infty}(X)$ is $\lambda$-outgoing, in the sense that (11.1) holds, and $\left(\Delta-\lambda^{2}\right) u \in x^{s} H_{\mathrm{sc}}^{M}(X)$ for some $s \geq \frac{1}{2}$ then $u \in x^{-\frac{1}{2}-\delta} H_{\mathrm{sc}}^{M+2}(X)$ for any $\delta>0$ and

$$
\mathrm{WF}_{\mathrm{sc}}^{M+2, s-1}(u) \subset R_{-}(\lambda) .
$$

Proof: The regularity arguments of the proof of Proposition 11 apply outside $R_{-}(\lambda)$, so (11.2) holds. Then (9.3) of Proposition 9 applies to give the additional regularity at $R_{-}(\lambda)$ from which it follows that $u \in x^{-\frac{1}{2}-\delta} H_{\mathrm{sc}}^{M+2}(X)$.

Thus, for a distribution with $\left(\Delta-\lambda^{2}\right) u \in H_{\mathrm{sc}}^{*, \frac{1}{2}}(X)$ the stronger form of the Sommerfeld radiation condition

$$
\mathrm{WF}_{\mathrm{sc}}^{*,-\frac{1}{2}}(u) \subset R_{-}(\lambda)
$$


follows from the weaker form (11.1). We now proceed to show that, with the assumption of more regularity on $\left(\Delta-\lambda^{2}\right) u$, the differential form of the Sommerfeld radiation condition:

$$
\mathrm{WF}_{\mathrm{sc}}^{*,-\frac{1}{2}}\left(\left(x^{2} D_{x}+\lambda\right) u\right)=\emptyset
$$

also follows from (11.3).

LEMMA 10. If, in addition to the hypotheses of Lemma 9, it is assumed that $\left(\Delta-\lambda^{2}\right) u \in x^{s} H_{\mathrm{sc}}^{M}(X)$ for some $s \geq 5 / 2$ then the differential form of the Sommerfeld radiation condition:

$$
\mathrm{WF}_{\mathrm{sc}}^{* \frac{1}{2}-\delta}\left(\left(x^{2} D_{x}-\lambda\right) u\right)=\emptyset \forall \delta>0,
$$

and hence (11.4), also holds.

Proof: Consider $v=\widetilde{\Delta}_{0} u$ where $\widetilde{\Delta}_{0}$ is the special extension of the boundary Laplacian discussed before Lemma 4 . From that lemma, the assumption that $(\Delta-$ $\left.\lambda^{2}\right) u \in x^{s} H_{\mathrm{sc}}^{M}(X)$ and the conclusion of Lemma 9 it follows that

$$
\mathrm{WF}_{\mathrm{sc}}^{M-2, s-1}\left(\left(\Delta-\lambda^{2}\right) v\right) \subset R_{-}(\lambda),\left(\Delta-\lambda^{2}\right) v \in \bigcap_{\delta>0} x^{-\frac{1}{2}-\delta} H_{\mathrm{sc}}^{M-1}(X) .
$$

Since $\mathrm{WF}_{\mathrm{sc}}^{*, s-1}(u) \cap R_{+}(\lambda)=\emptyset$ and $s-3 \geq \frac{1}{2}$ by assumption it follows that $v$ itself satisfies (11.1). From this we conclude that

$$
\widetilde{\Delta}_{0} u \in x^{-\frac{3}{2}-\delta} H_{\mathrm{sc}}^{M+1}(X) .
$$

Now observe from (3.4) that $\Delta=x\left(\left(x D_{x}\right)^{2}+i(n+1) x D_{x}-n\right) x+x \widetilde{\Delta}_{0} x+$ $x \operatorname{Diff}_{\mathrm{sc}}^{2}(X)$ so it follows from (11.7) that

$$
\left(\left(x^{2} D_{x}\right)^{2}-\lambda^{2}\right) u \in x^{\frac{1}{2}-\delta} H_{\mathrm{sc}}^{M+1}(X) .
$$

The factor $x^{2} D_{x}-\lambda \in \operatorname{Diff}_{\mathrm{sc}}^{1}(X)$ of this ordinary differential operator is elliptic near $R_{-}(\lambda)$ so the stronger form of (11.4), (11.5), in fact follows.

REMARK 2. Lemma 9 and Lemma 10 also hold if both signs are reversed.

The rather strange number $5 / 2$ in Lemma 10 is a figment of the method used, since we commute a full power of $\widetilde{\Delta}_{0}$ through the equation. If we use instead the cusp calculus, which we do not take the time to discuss, the following more natural result can be deduced in essentially the same way:

Lemma 11. If, in addition to the hypotheses of Lemma 9, it is assumed that $\left(\Delta-\lambda^{2}\right) u \in x^{s} H_{\mathrm{sc}}^{M}(X)$ for some $s>\frac{1}{2}$ then the differential form, (11.5), of the Sommerfeld radiation condition, and hence (11.4), also holds. 


\section{Section 12: Asymptotic expansions.}

If we assume more regularity for $\left(\Delta-\lambda^{2}\right) u$, with $u \lambda$-outgoing, then it follows that $u$ has a complete asymptotic expansion, which we write in the form

$$
x^{-\frac{n-1}{2}} e^{-\frac{i \lambda}{x}} u \in \mathcal{C}^{\infty}(X) .
$$

This regularity statement can be written more conventionally in terms of an asymptotic expansion (just the Taylor series) as $x \downarrow 0$ with respect to a product decomposition of $X$ near the boundary:

$$
u \sim e^{\frac{i \lambda}{x}} \times \sum_{j=0}^{\infty} x^{\frac{n-1}{2}+j} a_{j}(\omega), a_{j} \in \mathcal{C}^{\infty}(\partial X)
$$

Proposition 12. If $0 \neq \lambda \in \mathbb{R}$ and $u \in \mathcal{C}^{-\infty}(X)$ is $\lambda$-outgoing, in the sense that (11.1) holds, and satisfies $\left(\Delta-\lambda^{2}\right) u \in \dot{\mathcal{C}}^{\infty}(X)$ then $u$ satisfies (12.1). Conversely, given $g \in \mathcal{C}^{\infty}(\partial X)$ there exists $w \in \mathcal{C}^{\infty}(X)$ with $w_{\uparrow \partial X}=g$ such that $u=x^{\frac{(n-1)}{2}} \exp (i \lambda / x) w$ satisfies $\left(\Delta-\lambda^{2}\right) u \in \dot{\mathcal{C}}^{\infty}(X)$; moreover $w$ is determined by $g$ up to a term in $\dot{\mathcal{C}}^{\infty}(X)$.

PROOF: We first show the existence of a formal solution as in (12.1) or (12.2) with given leading term $a_{0}=g$. Indeed, using the form (3.4) of the Laplacian a simple computation shows that if $b \in \mathcal{C}^{\infty}(X)$ then

$$
\left(\Delta-\lambda^{2}\right) x^{p} e^{(i \lambda / x)} b=x^{p+1} e^{(i \lambda / x)} g, g \in \mathcal{C}^{\infty}(X), g=i \lambda(2 p-n+1) b+O(x) .
$$

This allows the formal series (12.2) to be constructed as a formal solution to the eigenvalue problem. Summation of the Taylor series for $u$ then gives a function $u$ with $\left(\Delta-\lambda^{2}\right) u \in \dot{\mathcal{C}}^{\infty}(X)$. The uniqueness, up to a term in $\dot{\mathcal{C}}^{\infty}(X)$ of the formal solution with a given leading coefficient follows from Proposition 11, since the difference of two such solution lies in $L_{\mathrm{sc}}^{2}(X)$.

Conversely suppose that $u$ satisfies (11.1) and $f=\left(\Delta-\lambda^{2}\right) u \in \dot{\mathcal{C}}^{\infty}(X)$. The argument in Lemma 10 can then be iterated. From Proposition 10 it follows directly that $\mathrm{WF}_{\mathrm{sc}}(u) \subset R_{-}(\lambda)$. Commuting $\widetilde{\Delta}_{0}$ through $\Delta-\lambda^{2}$, Lemma 4 shows that $\left[\widetilde{\Delta}_{0}, \Delta\right] \in \operatorname{Diff}_{\mathrm{sc}}^{3}(X)$, so $\left(\Delta-\lambda^{2}\right) \widetilde{\Delta}_{0} u \in H_{\mathrm{sc}}^{\infty,-\frac{1}{2}-\delta}(X)$ for any $\delta>0$. Then Proposition 9 shows that $\widetilde{\Delta}_{0} u \in H_{\mathrm{sc}}^{\infty,-\frac{3}{2}-\delta}(X)$ for any $\delta>0$. Since we have not developed the cusp calculus, to get the natural interpolation estimates we use the pseudodifferential operator calculus on the fibres, $\partial X$, of (2.1). From this we deduce

$$
\operatorname{Diff}_{\mathrm{c}}^{2}(X) u \subset H_{\mathrm{sc}}^{\infty,-\frac{3}{2}-\delta}(X) \text { and } \operatorname{Diff}_{\mathrm{c}}^{1}(X) u \subset H_{\mathrm{sc}}^{\infty,-1-\delta}(X) .
$$

Returning to the identity $\left(\Delta-\lambda^{2}\right)\left(\widetilde{\Delta}_{0} u\right)=\left[\Delta, \widetilde{\Delta}_{0}\right] u+\widetilde{\Delta}_{0} f$ it follows from (3.15) and (12.4) that $\left(\Delta-\lambda^{2}\right)\left(\widetilde{\Delta}_{0} u\right) \subset H_{\mathrm{sc}}^{\infty, \frac{1}{2}-\delta}(X)$, improving the previous estimate. 
Again applying Proposition 9 it now follows that $\widetilde{\Delta}_{0} u \in H_{\mathrm{sc}}^{\infty,-\frac{1}{2}-\delta}(X)$ and then (12.4) is replaced by

$$
\operatorname{Diff}_{\mathrm{c}}^{2}(X) u \subset H_{\mathrm{sc}}^{\infty,-\frac{1}{2}-\delta}(X) \forall \delta>0 .
$$

This two-step argument can be used inductively over $k$. Thus we can suppose inductively that (12.5) has been improved to

$$
\operatorname{Diff}_{\mathrm{c}}^{2(k-1)}(X) u \subset H_{\mathrm{sc}}^{\infty,-\frac{1}{2}-\delta}(X) \forall \delta>0 .
$$

It follows from Lemma 4 that

$$
\left[\widetilde{\Delta}_{0}^{k}, \Delta\right] \in x^{2} \operatorname{Diff}_{\mathrm{sc}}^{1}(X) \cdot \operatorname{Diff}_{\mathrm{c}}^{2 k}(X) \subset \operatorname{Diff}_{\mathrm{sc}}^{3}(X) \cdot \operatorname{Diff}_{\mathrm{c}}^{2(k-1)}(X) .
$$

For all $k$ we know that $\mathrm{WF}_{\mathrm{sc}}\left(\widetilde{\Delta}_{0}^{k} u\right) \subset R_{-}(\lambda)$ and it follows from (12.6) and (12.7) that $\left(\Delta-\lambda^{2}\right)\left(\widetilde{\Delta}_{0}^{k} u\right) \in H_{\mathrm{sc}}^{\infty,-\frac{1}{2}-\delta}(X)$. Thus the argument proceeds as for $k=1$ to give (12.6) for $k+1$ and hence for all $k$.

Thus $\widetilde{\Delta}_{0}^{k} u \in H_{\mathrm{sc}}^{\infty,-\frac{1}{2}-\delta}(X)$ for all $k$ and any $\delta>0$. For a product decomposition (2.1) this means that $u$ is a $\mathcal{C}^{\infty}$ function on $\partial X$ with values in $x^{\frac{1}{2}(n-1)-\delta} L_{\mathrm{b}}^{2}([0, \epsilon))$, where $L_{\mathrm{b}}^{2}([0, \epsilon))$ is the $L^{2}$ space with respect to $d x / x$. Now (3.12) shows that, near the boundary,

$$
x I(Q) x u-\lambda^{2} u \in x^{2} \operatorname{Diff}_{\mathrm{c}}^{2}(X) u+\dot{\mathcal{C}}^{\infty}(X) \subset \mathcal{C}^{\infty}\left(\partial X ; x^{2+\frac{1}{2}(n-1)-\delta} L_{\mathrm{b}}^{2}([0, \epsilon))\right) .
$$

Setting $u=x^{(n-1 / 2} v$ it follows that

$$
\left(x^{2} D_{x}-\lambda\right)\left(x^{2} D_{x}+\lambda\right) v \in \mathcal{C}^{\infty}\left(\partial X ; x^{2-\delta} L_{\mathrm{b}}^{2}([0, \epsilon))\right) .
$$

The first factor is elliptic on the wavefront set of $v$ so can be removed. It then follows by integration that

$v=\exp (i \lambda / x) a_{0}+v^{\prime}$ with $a_{0} \in \mathcal{C}^{\infty}(\partial X)$ and $v^{\prime} \in \mathcal{C}^{\infty}\left(\partial X ; x^{1+\frac{1}{2}(n-1)-\delta} L_{\mathrm{b}}^{2}([0, \epsilon))\right)$.

If $u^{\prime}$ is the formal solution with leading coefficient $a_{0}$ then $u^{\prime \prime}=u-u^{\prime} \in H_{\mathrm{sc}}^{\infty, 0}(X)$ satisfies $\left(\Delta-\lambda^{2}\right) u^{\prime \prime} \in \dot{\mathcal{C}}^{\infty}(X)$. Then Proposition 11 shows that $u^{\prime \prime} \in \dot{\mathcal{C}}^{\infty}(X)$, so $u$ has an asymptotic expansion (12.2).

The complex-conjugate of a formal solution is also a formal solution, with the sign of the phase reversed. Combining two such solutions we can produce more general formal solutions:

$$
u \sim e^{-\frac{i \lambda}{x}} \times \sum_{j=0}^{\infty} x^{\frac{n-1}{2}+j} a_{j}^{+}(\omega)+e^{\frac{i \lambda}{x}} \times \sum_{j=0}^{\infty} x^{\frac{n-1}{2}+j} a_{j}^{-}(\omega), a_{j}^{ \pm} \in \mathcal{C}^{\infty}(\partial X)
$$


where $a_{0}^{ \pm}$may be independently prescribed; they then determine, by local formulæ, all the other coefficients. In particular for $u$ as in (12.11)

$$
\mathrm{WF}_{\mathrm{sc}}^{*,-\frac{1}{2}}(u)=\left(R _ { - } ( \lambda ) \cap \pi ^ { * } ( \operatorname { s u p p } ( a _ { 0 } ^ { - } ) ) \cup \left(R_{+}(\lambda) \cap \pi^{*}\left(\operatorname{supp}\left(a_{0}^{+}\right)\right)\right.\right.
$$

where $\pi:{ }^{\text {sc }} T_{\partial X}^{*} X \longrightarrow \partial X$ is the bundle projection.

\section{Section 13: Boundary pairing.}

As the 'boundary data' of a boundary problem the leading coefficients, $a_{0}^{ \pm}$, in the asymptotic expansion of the formal solution in (12.11) are linked by a pairing relation.

Proposition 13. If $u_{i} \in \mathcal{C}^{\infty}\left(\stackrel{\circ}{X} ;{ }^{\mathrm{sc}} \Lambda^{k}\right)$ for $i=1,2$ satisfy $\left(\Delta-\lambda^{2}\right) u_{i}=f_{i} \in$ $\dot{\mathcal{C}}^{\infty}\left(X ;{ }^{\mathrm{sc}} \Lambda^{k}\right)$ and have asymptotic expansions (12.2) with leading coefficients $b_{i}^{ \pm}=$ $a_{0, i}^{ \pm} \in \mathcal{C}^{\infty}\left(\partial X ;{ }^{\mathrm{sc}} \Lambda^{k}\right)$ then

$$
-2 i \lambda \int_{\partial X}\left(\left\langle b_{1}^{+}, b_{2}^{+}\right\rangle-\left\langle b_{1}^{-}, b_{2}^{-}\right\rangle\right) d h=\int_{X}\left(\left\langle u_{1}, f_{2}\right\rangle-\left\langle f_{1}, u_{2}\right\rangle\right) d g .
$$

Proof: Choose a cutoff function $\phi \in \mathcal{C}^{\infty}(\mathbb{R})$ with $\phi(t)=0$ in $t<1$ and $\phi(t)=1$ in $t>2$. Then

$$
\begin{gathered}
\int_{X}\left(\left\langle u_{1}, f_{2}\right\rangle-\left\langle f_{1}, u_{2}\right\rangle\right) d g \\
=\lim _{\epsilon \downarrow 0} \int_{X}\left(\left\langle\phi\left(\frac{x}{\epsilon}\right) u_{1}, f_{2}\right\rangle-\left\langle f_{1}, \phi\left(\frac{x}{\epsilon}\right) u_{2}\right\rangle\right) d g \\
=\lim _{\epsilon \downarrow 0} \int_{X}\left\langle\left[\Delta, \phi\left(\frac{x}{\epsilon}\right)\right] u_{1}, u_{2}\right\rangle d g .
\end{gathered}
$$

From Lemma 3 it follows that the commutator $\left[\Delta, \phi\left(\frac{x}{\epsilon}\right)\right]$ is a first order differential operator of the form

$$
\frac{x}{\epsilon} \phi^{\prime}\left(\frac{x}{\epsilon}\right) x^{2} A+\frac{x}{\epsilon} \phi^{\prime \prime}\left(\frac{x}{\epsilon}\right) x^{2} g, A \in \operatorname{Diff}_{\mathrm{b}}^{1}(X) \text { and } g \in \mathcal{C}^{\infty}(X) .
$$

Thus, in terms of the product decomposition near the boundary, $A=q\left(x D_{x}\right)+V+h$ where $V x=0$ and $q$ and $h$ are smooth functions. Applying this to $u_{1}$ gives

$$
A u_{1}=q \lambda e^{-i \frac{i \lambda}{x}} x^{\frac{n-1}{2}-1} b_{1}^{+}-\lambda e^{i \frac{i \lambda}{x}} x^{\frac{n-1}{2}-1} b_{1}^{-}+O\left(x^{\frac{n-1}{2}}\right) .
$$


Inserting this into (13.3) shows that the error term in (13.4) disappears in the limit $\epsilon \downarrow 0$. From (3.4) it follows that $q=-2 i+O(x)$, where again the error term disappears in the limit. Thus

$$
\begin{gathered}
\lim _{\epsilon \downarrow 0} \int_{X}\left\langle\left[\Delta, \phi\left(\frac{x}{\epsilon}\right)\right] u_{1}, u_{2}\right\rangle d g \\
=\lim _{\epsilon \downarrow 0}-2 i \lambda \int_{\partial X} \int_{0}^{1} \phi^{\prime}\left(\frac{x}{\epsilon}\right)\left(e^{-i \frac{i \lambda}{x}} b_{1}^{+}-\lambda e^{i \frac{i \lambda}{x}} b_{1}^{-}\right)\left(e^{+i \frac{i \lambda}{x}} \overline{b_{2}^{+}}+e^{-i \frac{i \lambda}{x}} \overline{b_{1}^{-}}\right) \frac{d x}{\epsilon} d h \\
=-2 i \lambda \int_{\partial X}\left(b_{1}^{+} \overline{b_{2}^{+}}-b_{1}^{-} \overline{b_{2}^{-}}\right) d h .
\end{gathered}
$$

The cross terms in (13.5) disappear since they reduce to the Fourier transform of $\phi^{\prime}$, which is rapidly decreasing. This proves (13.1).

\section{Section 14: Limiting absorption principle.}

Now we can easily prove a weak form of the limiting absorption principle for the Laplacian of a scattering metric.

Proposition 14. If $0 \neq \lambda \in \mathbb{R}$ then for each $f \in \dot{\mathcal{C}}^{\infty}(X)$ the limit

$$
u_{ \pm}=\lim _{ \pm t \downarrow 0}\left(\Delta-(\lambda+i t)^{2}\right)^{-1} f
$$

exists in $x^{-\frac{1}{2}-\delta} H_{\mathrm{sc}}^{\infty}(X)$ for each $\delta>0$ and is such that $x^{-\frac{n-1}{2}} \exp (\mp i \lambda / x) u_{ \pm} \in$ $\mathcal{C}^{\infty}(X)$ is the unique solution of $\left(\Delta-\lambda^{2}\right) u=f$ satisfying the weak Sommerfeld radiation condition

$$
\mathrm{WF}_{\mathrm{sc}}^{*,-\frac{1}{2}}\left(u_{ \pm}\right) \cap R_{ \pm}(\lambda)=\emptyset .
$$

Proof: For the sake of definiteness, we shall take $t \geq 0$ throughout.

We start with the uniqueness. If $u \in \mathcal{C}^{-\infty}(X)$ satisfies $(14.2)$ and $\left(\Delta-\lambda^{2}\right) u=0$ then it follows from Proposition 12 that $u$ has an asymptotic expansion as in (12.2). Applying (13.1) with $u_{1}=u_{2}=u$ the right side vanishes identically and the left reduces to a non-vanishing multiple of $\left\|a_{0}\right\|^{2}$. Thus $a_{0} \equiv 0$. The uniqueness result in Proposition 11 therefore applies and shows that $u \equiv 0$.

To show the existence, consider $u_{t}=\left(\Delta-(\lambda+i t)^{2}\right)^{-1} f$. Thus $u_{t} \in \dot{\mathcal{C}}^{\infty}(X)$ by the results of $\S 6$. Choosing $\delta>0$, suppose that $u_{t}$ is not bounded in $x^{-\frac{1}{2}-\delta} L_{\mathrm{sc}}^{2}(X)$ as $t \downarrow 0$. Thus for some set $G \subset(0,1)$, with 0 in its closure, $\left\|u_{t}\right\|_{0,-\frac{1}{2}-\delta} \rightarrow \infty$ as $t \downarrow 0$ in $G$; this means $v_{t}=u_{t} /\left\|u_{t}\right\|_{0,-\frac{1}{2}-\delta}$, is bounded in $x^{-\frac{1}{2}-\delta} L_{\mathrm{sc}}^{2}(X)$ and satisfies $\left(\Delta-(\lambda+i t)^{2}\right) v_{t}=g_{t}=f /\left\|u_{t}\right\|_{0,-\frac{1}{2}-\delta} \rightarrow 0$ in $\dot{\mathcal{C}}^{\infty}(X)$. The elliptic estimates of the 
scattering calculus show that for any orders $\mathrm{WF}_{\mathrm{sc}, L^{\infty}(G)}^{M, L}\left(u_{t}\right)$ is contained in $\Sigma_{\mathrm{sc}}(\lambda)$. The estimate (9.12) applies and shows that for any $s \geq-\frac{1}{2}$

$$
\mathrm{WF}_{\mathrm{sc}, L^{\infty}(G)}^{*, s}\left(v_{t}\right) \cap R_{+}(\lambda)=\emptyset .
$$

Since a neighbourhood of $R_{+}(\lambda)$ is disjoint from the wavefront set the uniform propagation result in Proposition 8 shows that

$$
\mathrm{WF}_{\mathrm{sc}, L \infty(G)}^{*, s}\left(v_{t}\right) \subset R_{-}(\lambda) \forall s \geq-\frac{1}{2} .
$$

Finally then the uniform estimate of Proposition 9 implies that

$$
\mathrm{WF}_{\mathrm{sc}, L^{\infty}(G)}^{*, s}\left(v_{t}\right) \cap R_{-}(\lambda)=\emptyset \forall s<-\frac{1}{2} .
$$

Thus $v_{t}$ is bounded in $x^{-\frac{1}{2}-\epsilon} H_{\mathrm{sc}}^{M}(X)$ for every $M$ and any $\epsilon>0$. The compactness of the inclusion of $x^{r} H_{\mathrm{sc}}^{M}(X)$ in $x^{r^{\prime}} H_{\mathrm{sc}}^{M^{\prime}}(X)$ when $r>r^{\prime}$ and $M>M^{\prime}$ shows that, as $t \downarrow 0$ in $G$, each subsequence of $v_{t}$ must have strongly convergent subsequence in $x^{-\frac{1}{2}-\delta} L_{\mathrm{sc}}^{2}(X)$. The limit, $v$, of such a sequence satisfies $\left(\Delta-\lambda^{2}\right) v=0$ and $(14.2)$, so vanishes by the uniqueness discussed above. This implies that the sequence $v_{t} \rightarrow 0$ as $t \downarrow 0$, contradicting the definition in which $\left\|v_{t}\right\|=1$. Thus the assumption that $\left\|u_{t}\right\|$ is unbounded along a subsequence is false.

Thus we conclude that for $t \in(0,1)$ the family $u_{t}$ is bounded in $x^{-\frac{1}{2}-\delta} L_{\mathrm{sc}}^{2}(X)$ for any $\delta>0$. The same regularity analysis as just made now shows that any subsequence of $u_{t}$, along which $t \rightarrow 0$, has a subsequence strongly convergent in $x^{-\frac{1}{2}-\epsilon} L_{\mathrm{sc}}^{2}(X)$ for any $\epsilon>0$. The limit satisfies $\left(\Delta-\lambda^{2}\right) u_{+}=f$ and (14.2) and so is unique, by the discussion above. Thus in fact $u_{t} \rightarrow u_{+}$as indicated.

Although we do not take the space to do so, it is straightforward to extend this result to get mapping properties of the limit $(\Delta-(\lambda \pm i 0))^{-1}$ on the scattering Sobolev spaces.

\section{Section 15: The scattering matrix.}

Proposition 15. For each $0 \neq \lambda \in \mathbb{R}$ and $g \in \mathcal{C}^{\infty}(\partial X)$ there is a unique solution to $\left(\Delta-\lambda^{2}\right) u=0$ of the form (12.11) with $a_{0}^{-}=g$.

ProOF: Let $u^{\prime}$ be a formal solution, as in (12.2) with leading coefficient $g$. Thus $\left(\Delta-\lambda^{2}\right) u^{\prime}=f \in \dot{\mathcal{C}}^{\infty}(X)$ so by Proposition 14 there is a unique solution to $(\Delta-$ $\left.\lambda^{2}\right) u^{\prime \prime}=-f$ such that $\overline{u^{\prime \prime}}$ has an asymptotic expansion (12.2). Thus $u=u^{\prime}+u^{\prime \prime}$ is an eigenfunction with an expansion (12.11), with $a_{0}^{-}=g$. Uniqueness follows from Proposition 14.

Definition 5. The scattering matrix at any real, non-zero, energy is the operator $S(\lambda): \mathcal{C}^{\infty}(\partial X) \longrightarrow \mathcal{C}^{\infty}(\partial X)$ fixed by $S(\lambda) g=a_{0}^{+}$where $a_{0}^{+}$is the coefficient in (12.11) of the solution given by Proposition 15 . 
The symmetry between $\lambda$-incoming and $\lambda$-outgoing solutions shows that $S(\lambda)$ is invertible. In fact it follows by an application of Proposition 13 that $S(\lambda)$ extends to a unitary operator on $L^{2}(\partial X)$.

REMARK 3. These results hold, with proofs essentially unchanged, if the Laplacian for a scattering metric, $\Delta$, is replaced by $\Delta+V$ where $V \in x^{2} \mathcal{C}^{\infty}(X)$ is short-range. The proofs also hold, with inner products inserted at some points, for the action of $\Delta$ on forms.

In the standard, Euclidian, case the normalization of the Scatering matrix is slightly different, namely $S(\lambda)$ is replaced by $R \circ S(\lambda)$ where $R$ is the antipodal reflection in the sphere at infinity. There is no trivial analogue of this reflection in the general case of a scattering metric, since $\partial X$ can be quite general. We can think of $S(\lambda)$ as defined above as the 'absolute' scattering matrix, whereas the traditionally normalized version is a relative scattering matrix. The absolute scattering matrix for $\Delta+V$, takes the form $S(\lambda)=R+A(\lambda)$ where $A(\lambda)$ is a smoothing operator on the sphere, i.e. has smooth kernel. In the general case we anticipate that this statement should be replaced by the conjecture in the Introduction.

\section{Section 16: Long-range potentials.}

We shall briefly describe the changes that need to be made to treat the operator $\Delta+V$, where $\Delta$ is the Laplacian of a metric (1) and $V \in x \mathcal{C}^{\infty}(X)$ is real-valued and 'long-range.'

The main effect of such a perturbation can be seen directly on the formal solutions. For $\lambda \neq 0$ set

$$
\alpha=\alpha(\lambda)=\frac{1}{2 \lambda} \lim _{x \downarrow 0}(V / x) \in \mathcal{C}^{\infty}(\partial X) .
$$

Using the decomposition (2.1) to extend $\alpha$ off the boundary, (12.3) is replaced by:

$$
\begin{gathered}
\left(\Delta+V-\lambda^{2}\right)\left((\log x)^{r} x^{i \alpha+p} e^{(i \lambda / x)} b\right) \\
=x^{i \alpha+p+1} e^{(i \lambda / x)}\left(\sum_{s \leq r}(\log x)^{s} g_{s}+x \sum_{s \leq r+2}(\log x)^{s} g_{s}^{\prime}\right), \\
g_{r}=i \lambda(2 p-n+1) b, g_{s}, g_{s}^{\prime} \in \mathcal{C}^{\infty}(X) .
\end{gathered}
$$

Taking $r=0$ and $p=\frac{1}{2}(n-1)$ gives the leading term of a formal solution. Usin (16.1) to solve away the error terms iteratively shows that there is an asymptotic solution

$$
\begin{gathered}
u \sim e^{\frac{i \lambda}{x}} \times \sum_{j=0}^{\infty} \sum_{r \leq 2 j} x^{\frac{n-1}{2}+i \alpha+j}(\log x)^{r} a_{j, r}(\omega), \\
a_{j, r} \in \mathcal{C}^{\infty}(\partial X)
\end{gathered}
$$


with $a_{0,0}$ prescribed. This more complicated asymptotic series can still be summed asymptotically to give $u \in H_{\mathrm{sc}}^{\infty,-\frac{1}{2}-\delta}(X)$ of the form $\exp (i \lambda / x) v$ where $v$ is conormal to the boundary of $X$ and such that $\left(\Delta+V-\lambda^{2}\right) u \in \dot{\mathcal{C}}^{\infty}(X)$.

With this minor modification on the form of the limit Proposition 14 again holds and similarly for Proposition 15. That is, for each $0 \neq \lambda \in \mathbb{R}$ and $g \in \mathcal{C}^{\infty}(\partial X)$ there is a unique solution of $\left(\Delta+V-\lambda^{2}\right) u=0$ which is of the form

$$
u=e^{\frac{i \lambda}{x}} x^{\frac{n-1}{2}+i \alpha} g+e^{-\frac{i \lambda}{x}} x^{\frac{n-1}{2}-i \alpha+j} g^{\prime}+L_{\mathrm{sc}}^{2}(X) \text { with } g^{\prime} \in \mathcal{C}^{\infty}(\partial X)
$$

and then $S(\lambda): g \longmapsto g^{\prime}$ is the (absolute) scattering matrix. It is again unitary.

\section{ApPENDix A. B-GEOMETRY}

Before proceeding to describe the microlocalization of the algebra of scattering differential operators, Diff* sc $^{*}(X)$, to an algebra of pseudodifferential operators we describe, without proof, some properties of manifolds with corners and the maps between them, these results are used in the analysis below. For a more general, and complete, treatment of manifolds with corners see [8].

\section{Section 17: Manifolds with corners.}

By a manifold with corners we mean a topological manifold with boundary which has a $\mathcal{C}^{\infty}$ structure $\mathcal{C}^{\infty}(X)$ which arises from local coordinates $x_{1}, \ldots, x_{k}, y_{1}, \ldots, y_{n-k}$ where $x_{j} \geq 0$, and for which the boundary is a finite union of closed, connected, embedded hypersurfaces. Thus the boundary can be written as a union

$$
\partial X=\bigcup_{H \in M_{1}(X)} H
$$

where there exists a 'boundary defining function,' $\rho_{H} \in \mathcal{C}^{\infty}(X)$, for each boundary hypersurface $H \in M_{1}(X)$. Each $\rho_{H} \geq 0$ is such that $d \rho_{H} \neq 0$ on $H=\left\{\rho_{H}=0\right\}$. Locally this is not an additional condition, since the $\rho$ 's can be taken to be the $x_{j}$. Globally the existence of $\rho_{H}$ means that the boundary hypersurface $H$ cannot have self-intersections at corners. The boundary faces of $X$ are the connected components of the intersections of the boundary hypersurfaces. We write $M^{\prime}(X)=$ $M_{1} \cup \cdots \cup M_{n}(X)$ for the collection of all boundary faces, where $M_{k}(X)$ is the set of boundary faces of codimension $k$.

A map $f: X \longrightarrow Y$ between manifolds with corners is smooth if $f^{*}: \mathcal{C}^{\infty}(Y) \longrightarrow$ $\mathcal{C}^{\infty}(X)$. It is said to be a b-map if it behaves well with respect to the bounday hypersurfaces in the sense that if $\rho_{G}^{\prime}$, for $G \in M_{1}(Y)$, are boundary defining functions for the boundary hypersurfaces of $Y$, then

$$
\begin{gathered}
f^{*} \rho_{G}^{\prime}=b_{G} \prod_{H \in M_{1}(X)} \rho_{H}^{e(H, G)} \text { with } 0<b_{G} \in \mathcal{C}^{\infty}(X) \\
\text { or } f^{*} \rho_{G}^{\prime} \equiv 0 .
\end{gathered}
$$


If the second case does not occur then $f$ is said to be an interior b-map. The 'boundary exponents' $e(H, G)$ of a b-map are necessarily non-negative integers (since it is assumed to be smooth).

For manifolds with corners there is, apart from the usual tangent bundle $T X$, a 'b-tangent bundle' ${ }^{\mathrm{b}} T X$. As with the standard tangent bundle this is defined from vector fields. Thus let $\mathcal{V}_{\mathrm{b}}(X)$ be the space of all vector fields on $X$ which are tangent to all boundaries. In local coordinates $V \in \mathcal{V}_{\mathrm{b}}(X)$ if it is of the form

$$
V=a_{1} x_{1} \frac{\partial}{\partial x_{1}}+\cdots+a_{k} x_{k} \frac{\partial}{\partial x_{k}}+a_{k+1} \frac{\partial}{\partial y_{1}}+\cdots+a_{n} \frac{\partial}{\partial y_{n-k}}
$$

with the coefficients general smooth functions of $x$ and $y$. This means that the elements of $\mathcal{V}_{\mathrm{b}}(X)$ form all the sections of a vector bundle, ${ }^{\mathrm{b}} T X$, over $X$. More formally the fibre at any point $p$ is

$$
{ }^{\mathrm{b}} T_{p} X=\mathcal{V}_{\mathrm{b}}(X) / \mathcal{I}_{p} \cdot \mathcal{V}_{\mathrm{b}}(X)
$$

where $\mathcal{I}_{p} \subset \mathcal{C}^{\infty}(X)$ is the ideal of functions vanishing at $p$.

\section{Section 18: Blow-up.}

A p-submanifold, $S$, of a manifold with corners, $X$, is a subset with the property that each of its points, $s$, has a coordinate neighbourhood with coordinates $x_{1}, \ldots, x_{k}, y_{1}, \ldots, y_{n-k}$ such that the boundary hypersurfaces of $X$ through $s$ are locally given by $x_{i}=0$ for $i=1, \ldots, k$ and in terms of which

$$
S=\left\{x_{i}=0, y_{j}=0, i=1, \ldots, k^{\prime}, j=1, \ldots, q\right\},
$$

where either $k^{\prime}$ or $q$ can vanish. The 'p' is short for product since there is a local decomposition of $X$ as the product of intervals and half-intervals in terms of which $S$ is a product. A boundary face is always a p-submanifold.

If $S \subset X$ is a closed p-submanifold then ' $X$ blown up along $S$ ' is a new manifold with corners obtained by introducing polar coordinates in the normal directions to $S$ at $S$. Thus $X$ is unchanged away from $S$ but $S$ itself is replaced by a new boundary hypersurface the 'front face' of the blow up. The blown-up manifold is denoted $[X ; S]$ it comes with a natural (blow-down) map $\beta[X ; S]:[X ; S] \longrightarrow X$ which is a diffeomorphism of the complement of $\mathrm{ff}[X ; S]$, the front face, onto the complement of $S$; it is a b-map. Smooth functions on $[X ; S]$ are simply (bounded) functions of $X$ which are smooth when expressed in terms of local polar coordinates in the normal directions to $S$.

The lift, $\beta^{*}[X ; S](C)$ of a closed subset $C \subset X$ under the blow up of $S$ is defined in case $C$ is the closure of $C \backslash S$ to be the closure in $[X ; S]$ of $\beta^{-1}(C \backslash S)$ and in case $C \subset S$ to be $\beta^{-1}(C)$; otherwise it is not defined. If $S_{1} \subset S_{2} \subset X$ are both closed 
p-submanifolds then $\beta^{*}\left[X ; S_{1}\right]\left(S_{2}\right) \subset\left[X ; S_{1}\right]$ and $\beta^{*}\left[X ; S_{2}\right]\left(S_{1}\right) \subset\left[X ; S_{2}\right]$ are also $\mathrm{p}$-submanifolds and there is a natural isomorphism

$$
\left[X ; S_{1} ; S_{2}\right] \equiv\left[X ; S_{2} ; S_{1}\right]
$$

where $\left[X ; S_{1} ; S_{2}\right]=\left[\left[X ; S_{1}\right] ; \beta^{*}\left[X ; S_{1}\right]\left(S_{2}\right)\right]$ etc.

Two p-submanifolds are said to be p-transversal if at each point of their intersection there is a local product decomposition, simultaneously for the manifold and for each of the submanifolds, such that each factor appears in at least one of the submanifolds. The commutativity result (18.1) again holds for two closed p-transversal p-submanifolds (but not more generally).

\section{Section 19: b-fibrations.}

For an interior b-map there is a naturally defined b-differential

$$
{ }^{\mathrm{b}} f_{*}:{ }^{\mathrm{b}} T_{p} X \longrightarrow{ }^{\mathrm{b}} T_{f(p)} Y
$$

and, as in the category of manifolds without boundary, we distinguish certain maps by properties of the differential. If the b-differential is always surjective, as a map (19.1), then $f$ is said to be a b-submersion. The blow-down map for the blow up of a boundary face is a b-submersion. At a boundary point of codimension $k$ the span of the $x_{i} \partial / \partial x_{i}$ forms a natural subspace, the b-normal space, ${ }^{\mathrm{b}} N_{p} X \subset{ }^{\mathrm{b}} T_{p} X$. For a b-map the b-differential always maps ${ }^{\mathrm{b}} N_{p} X$ into ${ }^{\mathrm{b}} N_{f(p)} Y$. If $f$ is a b-submersion and in addition the map

$$
{ }^{\mathrm{b}} f_{*}:{ }^{\mathrm{b}} N_{p} X \longrightarrow{ }^{\mathrm{b}} N_{f(p)} Y
$$

is also always surjective then $f$ is said to be a b-fibration. Such b-fibrations are important because of the properties of the push-forward map, i.e. integration over the fibres, defined by them. For a b-submersion the additional condition required for the map to be a b-fibration is combinatorial. It is equivalent to the requirement that no boundary hypersurface of $X$ be mapped by $f$ into the intersection of two (or more) boundary hypersurfaces of $Y$. A non-trivial blow-down map (i.e. except in the case of the blow up of a hypersurface) is not a b-fibration.

For a p-submanifold the relative b-tangent bundle is defined to be

$$
\begin{gathered}
{ }^{\mathrm{b}} T_{p}(S ; X)=\left\{v \in{ }^{\mathrm{b}} T_{p} X ; \exists V \in \mathcal{V}_{\mathrm{b}}(X ; S) \text { with } V(p)=v\right\} \\
\mathcal{V}_{\mathrm{b}}(X ; S)=\left\{V \in \mathcal{V}_{\mathrm{b}}(X) ; V \text { is tangent to } S\right\} .
\end{gathered}
$$

Notice that if $S$ is an interior p-submanifold, meaning that $k^{\prime}=0$, then this is just the closure in ${ }^{\mathrm{b}} T X$ of the ordinary tangent space of $S$ at its interior points. For a boundary p-submanifold the parts lying over it of the b-normal spaces to the boundary hypersurfaces which contain it are always in ${ }^{\mathrm{b}} T(S ; X)$. Thus its fibre 
dimension is actually larger than the dimension of $S$. In either case any section $v \in \mathcal{C}^{\infty}\left(S ;{ }^{\mathrm{b}} T(S ; X)\right)$ can be extended to an element $V \in \mathcal{V}_{\mathrm{b}}(X ; S)$ and conversely an element of $\mathcal{V}_{\mathrm{b}}(X)$ is in $\mathcal{V}_{\mathrm{b}}(X ; S)$ only if the section of $\mathcal{C}^{\infty}\left(X ;{ }^{\mathrm{b}} T X\right)$ it defines restricts to $S$ to be a section of ${ }^{\mathrm{b}} T(S ; X)$.

If $f: X \longrightarrow Y$ is an interior b-map, with b-differential ${ }^{\mathrm{b}} f_{*}$, then $S$ is said to be b-transversal to $f$ (or $f$ to be b-transversal to $S$ ) if

$$
{ }^{\mathrm{b}} T_{p}(S ; X) \oplus \operatorname{null}\left({ }^{\mathrm{b}} f_{*}\left\lceil{ }^{\mathrm{b}} T_{p} X\right)={ }^{\mathrm{b}} T_{p} X \quad \forall p \in S .\right.
$$

Lemma 12. If $S \subset B$ where $B \in M^{\prime}(X)$ is a boundary face of $X$ then the transversality of an interior b-map $f: X \longrightarrow Y$ to $S$ is equivalent to the transversality of $S$ as a submanifold of $B$ to $f_{B}$, defined as the interior b-map give by $f$ restricted to $B$ but with range space the smallest boundary face of $Y$ containing $f(B)$.

The following result involving this notion of tranversality gives a sufficient condition for a b-map defined by blow up to be a b-submersion.

Lemma 13. If $f: X \longrightarrow Y$ is a b-submersion between compact manifolds with boundary and $S \subset X$ is a p-submanifold which is b-transversal to $f$ then the composite map $f^{\prime}=f \circ \beta[X ; S]$ is a b-submersion from $[X ; S]$ to $Y$.

Similarly there is a sufficient condition for a b-fibration to lift under blow up to a b-fibration.

Proposition 16. If $f: X \longrightarrow Y$ is a b-fibration between compact manifolds with boundary and $S \subset X$ is a p-submanifold which is b-transversal to $f$ and such that $f(S)$ is not contained in any boundary fact of $Y$ of codimension 2 then the composite map $f^{\prime}=f \circ \beta[X ; S]$ is a b-fibration from $[X ; S]$ to $Y$.

\section{Section 20: Distributions and operations.}

In the main we are concerned with conormal distributions. Let $X$ be a compact manifold with corners and let $\mathfrak{a}$ be a multi-order in the sense of a map $\mathfrak{a}: M_{1}(X) \longrightarrow$ $\mathbb{R}$, so $\mathfrak{a}(H) \in \mathbb{R}$ for each boundary hypersurface $H$. Then $\rho^{\mathfrak{a}} L^{\infty}(X)$ is the space of (equivalence classes of) measurable functions on $X$ such that

$$
\rho^{-\mathfrak{a}} u \in L^{\infty}(X), \rho^{\mathfrak{a}}=\prod_{H \in M_{1}(X)} \rho_{H}^{\mathfrak{a}(H)} .
$$

The basic space of conormal distributions (the elements are functions) with multiorder $\mathfrak{a}$ is:

$$
\mathcal{A}^{\mathfrak{a}}(X)=\left\{u \in \rho^{\mathfrak{a}} L^{\infty}(X) ; \operatorname{Diff}_{\mathrm{b}}^{*}(X) u \subset \rho^{\mathfrak{a}} L^{\infty}(X)\right\} .
$$

If 0 stands for the multiorder assigning 0 to all boundary hypersurfaces then $\mathcal{C}^{\infty}(X) \subset \mathcal{A}^{0}(X)$. Clearly for any two multiorders $\rho^{\mathfrak{b}} \mathcal{A}^{\mathfrak{a}}(X)=\mathcal{A}^{\mathfrak{a}+\mathfrak{b}}(X)$. We can then say $u \equiv 0$ at $H$ for $u \in \mathcal{A}^{\mathfrak{a}}(X)$ if

$$
u \in \bigcap_{\mathfrak{b}_{j}} \mathcal{A}^{\mathfrak{b}_{j}}(X), \mathfrak{b}_{j}(G)=\mathfrak{a}(G) \forall G \neq H, \mathfrak{b}_{j}(H)=j .
$$


LEMMA 14. Under radial compactification of $\mathbb{R}^{n}$

$$
\mathrm{SP}^{*}: \mathcal{A}^{a}\left(\mathbb{S}_{+}^{n}\right) \longrightarrow S^{-a}\left(\mathbb{R}^{n}\right)
$$

is an isomorphism.

As well as these space we also consider spaces $I^{m, \mathfrak{a}}(X, S)$ of conormal distributions associated to a closed interior p-submanifold $S$; here $m$ is the order of the singularity across $S$ and $\mathfrak{a}$ is the multiorder at the boundary as before. These are a direct generalization of the conormal distributions introduced by Hörmander [4] in the case of a submanifold of a manifold without boundary. Away from $S$ an element of $I^{m, \mathfrak{a}}(X)$ must reduce to an element of $\mathcal{A}^{\mathfrak{a}}(X)$. Near $S$ but away from the boundary it must reduce to an element of $I^{m}(X ; S)$ as defined in the interior case, i.e.

$$
u=\int_{\mathbb{R}^{s}} e^{i y^{\prime} \cdot \eta^{\prime}} a\left(y^{\prime \prime}, \eta^{\prime}\right) d \eta^{\prime}
$$

where $y=\left(y^{\prime}, y^{\prime \prime}\right)$ are local coordinates with $S=\left\{y^{\prime}=0\right\}$ locally, $s$ is the codimension of $S$ and $a$ is a symbol of order $M=m-\frac{1}{2} s+\frac{1}{4} n, n=\operatorname{dim} X$ :

$$
\left|\left(D_{y^{\prime \prime}}\right)^{\gamma}\left(D_{\eta^{\prime}}\right)^{\beta} a\left(y^{\prime \prime}, \eta^{\prime}\right)\right| \leq C_{\beta, \gamma}\left(1+\left|\eta^{\prime}\right|\right)^{M-|\beta|}, \forall \beta \text { and } \gamma .
$$

Near a boundary point of $S$ of codimension $k$ in $X$ there are (by definition) product coordinates $x, y^{\prime}, y^{\prime \prime}$ where $x_{i} \geq 0$ locally, for $i=1, \ldots, k$ and the $y^{\prime}$ still define $S$. Then we require that (20.4) still hold with $a$ replaced by $a\left(x, y^{\prime \prime}, \eta^{\prime}\right)$ satisfying in place of (20.5) the estimates

$$
\left|\left(x D_{x}\right)^{\alpha}\left(D_{y^{\prime \prime}}\right)^{\gamma}\left(D_{\eta^{\prime}}\right)^{\beta} a\left(x, y^{\prime \prime}, \eta^{\prime}\right)\right| \leq C_{\alpha, \beta, \gamma} x^{\mathfrak{a}}\left(1+\left|\eta^{\prime}\right|\right)^{M-|\beta|}, \forall \alpha, \beta \text { and } \gamma
$$

Here $x D_{x}=\left(x_{1} D_{x_{1}}, x_{2} D_{x_{2}} \ldots, x_{k} D_{x_{k}}\right)$.

Lemma 14 shows that under radial compactification in the fibre, $\eta^{\prime}$, variables (20.6) reduces to the conormal estimates (20.1). That is, assuming it has compact support in $x$ and $y^{\prime \prime}, a$ satisfies (20.6) if and only if

$$
a=\mathrm{SP}_{\eta^{\prime}}^{*}\left(a^{\prime}\right), a^{\prime} \in \mathcal{A}^{\mathfrak{b}}\left([0, \infty)^{k} \times \mathbb{R}^{n-s-k} \times \mathbb{S}_{+}^{s}\right)
$$

where $\mathfrak{b}$ assigns order $-M$ to the boundary of $\mathbb{S}_{+}^{s}$ and the appropriate entry of $\mathfrak{a}$ to the boundaries $x_{i}=0$.

We refer to [9] for a treatment of the coordinate transformation and symbolic properties of these various space of conormal distributions and for a discussion of the pull-back and push-forward operations on conormal distributions. The importance of b-fibrations lies principally in the fact that under them integrable conormal densities with full asymptotic expansions push forward to have the same property. 


\section{Appendix B. ScAttering CAlculus}

We now outline a directly global approach to the scattering calculus defined in $\S 4$. As already noted the properties of the calculus are easily deduced from the general Weyl calculus of Hörmander. The present approach allows this calculus to be integrated more directly with others, including both the boundary ('b') and cusp ('c') calculi.

\section{Section 21: Stretched spaces.}

In the sense of the general approach to degenerate problems pursued in [8] the single space for the scattering calculus is just the compact manifold with boundary, $X$, itself. For simplicity of notation we shall assume that the boundary of $X$ is connected. To define the double space we start with the double space for the b-calculus:

$$
X_{\mathrm{b}}^{2}=\left[X^{2} ;(\partial X)^{2}\right]
$$

The diagonal lifts to a p-submanifold $\Delta_{\mathrm{b}} \subset X_{\mathrm{b}}^{2}$ which meets the boundary only in the interior of the front face for the blow up (21.1). The scattering double space is then defined to be

$$
X_{\mathrm{sc}}^{2}=\left[X_{\mathrm{b}}^{2} ; \partial \Delta_{\mathrm{b}}\right]
$$

Lemma 15. The diagonal lifts to a $p$-submanifold $\Delta_{\mathrm{sc}} \subset X_{\mathrm{sc}}^{2}$ which meets the boundary only at the front face for the blow up (21.2) and the stretched projections $\pi_{\mathrm{sc}, F}^{2}: X_{\mathrm{sc}}^{2} \longrightarrow X, F=L, R$, are $b$-fibrations which are transversal to the lifted diagonal and give a commutative diagram:

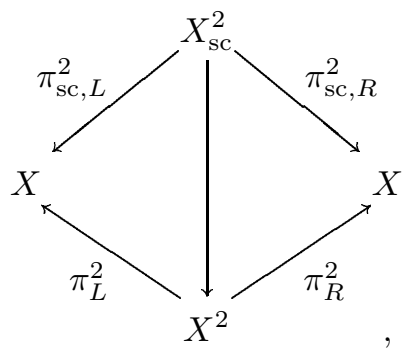

ProOF: The stretched projections are obtained by composition of the blow-down map from $X_{\mathrm{sc}}^{2}$ to $X^{2}$ with the projections $\pi_{F}^{2}$, for $F=L, R$. These are all b-maps, hence so are the stretched projections.

It is shown in [8] that the stretched projections, $\pi_{\mathrm{b}, R}^{2}$ and $\pi_{\mathrm{b}, L}^{2}$, from $X_{\mathrm{b}}^{2}$ to $X$ are both b-fibrations. In fact that they are b-submersions follows from Lemma 13 since they are the composites of the blow-down map for (21.1) and the projections, from 
$X^{2}$ to $X$, which are b-submersions transversal to $(\partial X)^{2}$. Since the range space $X$ is a manifold with boundary it follows automatically that they are b-fibrations.

The same argument shows that the stretched projections $\pi_{\mathrm{sc}, L}^{2}$ and $\pi_{\mathrm{sc}, R}^{2}$ are bfibrations. Since they are the composites of the blow-down map for (21.2) with $\pi_{\mathrm{b}, L}^{2}$ and $\pi_{\mathrm{b}, R}^{2}$ it suffices to check that these two maps are b-transversal to $\partial \Delta_{\mathrm{b}}$. In fact $\mathcal{V}_{\mathrm{b}}(X)$ lifts under either map to be transversal to $\Delta_{\mathrm{b}}$, so certainly the null space of either b-stretched projection is b-transversal to $\partial \Delta_{\mathrm{b}}$ in the sense of (19.4).

The commutativity of (21.3) follows directly from the definitions of the various maps. Similarly the fact that the diagonal lifts to a p-submanifold $\Delta_{\mathrm{sc}}$ follows from the lifting results of [8;Chapter 5]. Thus it only remains to show that $\pi_{\mathrm{sc}, L}^{2}$ and $\pi_{\mathrm{sc}, R}^{2}$

are transversal to this lifted diagonal. Consider the lift to $X_{\mathrm{sc}}^{2}$ of the Lie algebra $\mathcal{V}_{\mathrm{sc}}(X)$, i. e. $x \mathcal{V}_{\mathrm{b}}(X)$ for any boundary defining function $x$. As has already been noted the Lie algebra $\mathcal{V}_{\mathrm{b}}(X)$ lifts to $X_{\mathrm{b}}^{2}$ to span a Lie algebra $\mathcal{W}$ which is transversal to $\Delta_{\mathrm{b}}$. Since $\Delta_{\mathrm{b}}$ meets the boundary of $X_{\mathrm{b}}^{2}$ only in the interior of the front face for the blow up (21.1) it follows that $\mathcal{V}_{\mathrm{sc}}(X)$ lifts to span $\rho \mathcal{W} \subset \mathcal{V}_{\mathrm{b}}\left(X_{\mathrm{b}}^{2} ; \partial \Delta_{\mathrm{b}}\right)$ near $\partial \Delta_{\mathrm{b}}$. Certainly then $\mathcal{V}_{\mathrm{sc}}(X)$ lifts under $\pi_{\mathrm{sc}, L}^{2}$ or $\pi_{\mathrm{sc}, R}^{2}$ into $\mathcal{V}_{\mathrm{b}}\left(X_{\mathrm{sc}}^{2}\right)$. Moreover the transversality of $\mathcal{W}$ to $\Delta_{\mathrm{b}}$ implies the transversality of the lift of $\rho \mathcal{W}$ to the lift of $\Delta_{\mathrm{b}}$, i.e. to $\Delta_{\mathrm{sc}}$.

\section{Section 22: Scattering pseudodifferential operators.}

Using the definitions and properties of the sc-stretched product discussed in $\S 21$ we define the small calculus of sc-pseudodifferential operators and show that it coincides with the definition by localization given in $\S 4$. To define these operators we first examine the identity operator for the purposes of normalization. By the scattering density bundle we shall mean the weighted density bundle ${ }^{\mathrm{sc}} \Omega X=x^{-1-n} \Omega X$ where $\Omega X$ is the usual density bundle and $n=\operatorname{dim} X$. To reduce the number of different bundles involved to a minimum we shall consider the identity operator acting on the sc- $\frac{1}{2}$-density bundle ${ }^{\mathrm{sc}} \Omega^{\frac{1}{2}} X$.

Let $(x, y)$ be local coordinates near the boundary of $X$, with $x$ a boundary defining function and the $y_{j}$ for $j=1, \ldots, n$ tangential coordinates. The Schwartz kernel of Id is the half density

$$
\kappa(\mathrm{Id})=\delta\left(x-x^{\prime}\right) \delta\left(y-y^{\prime}\right) \frac{\left(x^{\prime}\right)^{\frac{1}{2}(n+2)}\left|d x d y d x^{\prime} d y^{\prime}\right|^{\frac{1}{2}}}{x^{\frac{1}{2}(n+2)}}
$$

where $(x, y)$ and $\left(x^{\prime} y^{\prime}\right)$ are these coordinates in the left and right factors of $X$. The support of $\kappa(\mathrm{Id})$ is contained in the diagonal and we wish to consider the form it takes when lifted from $X^{2}$ to $X_{\mathrm{sc}}^{2}$. Lifting first to $X_{\mathrm{b}}^{2}$ we can use as coordinates near $\partial \Delta_{\mathrm{b}}$ the variables $s=x / x^{\prime}, y, x^{\prime}$ and $y^{\prime}$. In terms of these coordinates $\kappa(\mathrm{Id})$ becomes

$$
\kappa(\mathrm{Id})=\delta(s-1) \delta\left(y-y^{\prime}\right) \frac{\left|d s d x^{\prime} d y d y^{\prime}\right|^{\frac{1}{2}}}{\left(x^{\prime}\right)^{\frac{1}{2}} s^{\frac{1}{2}(n+2)}}
$$


In a neighbourhood of $\partial \Delta_{\mathrm{sc}} \subset X_{\mathrm{sc}}^{2}$ the coordinates $X=(s-1) / x^{\prime}, Y=\left(y-y^{\prime}\right) / x^{\prime}$, $x^{\prime}$ and $y^{\prime}$ are valid and in terms of them

$$
\kappa(\mathrm{Id})=\delta(X) \delta(Y)\left(x^{\prime}\right)^{-\frac{1}{2}(n+2)}\left|d x^{\prime} d X d Y d y^{\prime}\right|^{\frac{1}{2}} .
$$

In view of this computation we define the kernel density bundle (for operators on sc-half-densities) by

$$
\mathrm{KD}_{\mathrm{sc}}^{\frac{1}{2}}=\rho_{\mathrm{sf}}^{-\frac{1}{2}(\operatorname{dim} X+1)} \Omega^{\frac{1}{2}}\left(X_{\mathrm{sc}}^{2}\right)
$$

where $\rho_{\mathrm{sf}} \in \mathcal{C}^{\infty}\left(X_{\mathrm{sc}}^{2}\right)$ is a defining function for the 'scattering front face' $\operatorname{sf}\left(X_{\mathrm{sc}}^{2}\right)$ which is the front face for the blow up (21.2).

Before defining the calculus of scattering pseudodifferential operators note that the lifts of the basic vector fields $x^{2} \partial_{x}$ and $x \partial_{y}$ from the left factor of $X^{2}$ to a neighbourhood of the interior of sf $\subset X_{\mathrm{sc}}^{2}$ are easily computed using the same coordinates. Thus near $\partial \Delta_{\mathrm{b}}$ in $X_{\mathrm{b}}^{2}$ they become

$$
x^{\prime} s^{2} \partial_{s}, x^{\prime} s \partial_{y}
$$

Lifting further to $X_{\mathrm{sc}}^{2}$ gives

$$
\left(1+x^{\prime} X\right)^{2} \partial_{X}, \partial_{Y}
$$

in the region of validity of the coordinates.

Using the kernel density bundle from (22.4) we now define the small calculus, with weight $l \in \mathbb{R}$ and order $m \in \mathbb{R}$ as given by the space of kernels

$$
\Psi_{\mathrm{scc}}^{m, l}\left(X ;{ }^{\mathrm{sc}} \Omega^{\frac{1}{2}}\right)=\left\{\kappa \in \mathcal{A}^{m, l}\left(X_{\mathrm{sc}}^{2}, \Delta_{\mathrm{sc}} ; \mathrm{KD}_{\mathrm{sc}}^{\frac{1}{2}}\right) ; \kappa \equiv 0 \text { at } \partial X_{\mathrm{sc}}^{2} \backslash \mathrm{sf}\left(X_{\mathrm{sc}}^{2}\right)\right\} .
$$

Here $\mathcal{A}^{m, l, \infty}\left(X_{\mathrm{sc}}^{2}, \Delta_{\mathrm{sc}}\right)$ is the space of conormal distributions on $X_{\mathrm{sc}}^{2}$ with respect to the lifted diagonal, as defined in $\S 20$. We define a smaller space by replacing these conormal distributions by the subspace $I_{\mathrm{os}}^{m}(X ; S)$ of 1-step polyhomogeneous ('classical') conormal distributions defined as in [4] by extension across the boundary (see [8]). In particular the elements of $I_{\mathrm{os}}^{k}\left(X_{\mathrm{sc}}^{2}, \Delta_{\mathrm{sc}}\right) \subset I^{k, 0}\left(X_{\mathrm{sc}}^{2}, \Delta_{\mathrm{sc}}\right)$ are $\mathcal{C}^{\infty}$ near boundary hypersurfaces of $X_{\mathrm{sc}}^{2}$ other than sf. Thus the vanishing in Taylor series at these boundary hypersurfaces, written $\kappa \equiv 0$ in (22.6), is well defined. Then we define, for $l \in \mathbb{Z}$,

$$
\Psi_{\mathrm{sc}}^{m, l}\left(X ;{ }^{\mathrm{sc}} \Omega^{\frac{1}{2}}\right)=\left\{\kappa \in \rho_{\mathrm{sf}}^{l} I_{\mathrm{os}}^{m}\left(X_{\mathrm{sc}}^{2}, \Delta_{\mathrm{sc}} ; \mathrm{KD}_{\mathrm{sc}}^{\frac{1}{2}}\right) ; \kappa \equiv 0 \text { at } \partial X_{\mathrm{sc}}^{2} \backslash \mathrm{sf}\left(X_{\mathrm{sc}}^{2}\right)\right\} .
$$

The blow-ups involved in the definition of $X_{\mathrm{sc}}^{2}$ are all of boundary p-submanifolds. Thus the spaces of extendable distributions on $X^{2}$ and $X_{\mathrm{sc}}^{2}$ are canonically isomorphic. In particular the kernels in (22.6) can be identified as the kernels of continuous linear operators

$$
A \in \Psi_{\mathrm{scc}}^{m, l}\left(X ;{ }^{\mathrm{sc}} \Omega^{\frac{1}{2}}\right) \Longrightarrow A: \dot{\mathcal{C}}^{\infty}\left(X ;{ }^{\mathrm{sc}} \Omega^{\frac{1}{2}}\right) \longrightarrow \mathcal{C}^{-\infty}\left(X ;{ }^{\mathrm{sc}} \Omega^{\frac{1}{2}}\right) .
$$

We simply have to show that these are precisely the operators defined by (4.1) and (4.2). 
Proposition 17. The definitions (22.6) and (22.7) give the same spaces of kernels as defined by localization in $\S 4$.

Proof: First we show that the two definitions coincide for $\mathbb{S}_{+}^{n}$, i.e. under SP acting in each factor the space of kernels fixed by (22.6) is mapped precisely to the space of kernels determined by (4.1) and (4.2). Consider an operator of this type on $\mathbb{R}^{n}$. The spaces of kernels are easily seen to be the same in the interior. Radial compactification is represented by the change of variable to $z=\theta / x, z^{\prime}=\theta^{\prime} / x^{\prime}$. Moreover integration by parts shows that as $x \downarrow 0$ with $x^{\prime}>0$, or $x^{\prime} \downarrow 0$ with $x>0$, or in $\theta \neq \theta^{\prime}$ as both $x \downarrow 0$ and $x^{\prime} \downarrow 0$ the kernel

$$
A^{\prime}=\frac{1}{(2 \pi)^{n}} \int e^{i\left(\frac{\theta}{x}-\theta^{\prime} x^{\prime}\right) \cdot \zeta} a_{L}(\theta / x, \zeta) d \zeta
$$

is $\mathcal{C}^{\infty}$ and vanishes rapidly with all derivatives. Thus we may assume that $\theta-\theta^{\prime}$ is small and consider the behaviour as $x, x^{\prime} \downarrow 0$.

We first consider the lift of $(22.9)$ to the space $(21.1)$, for $X=\mathbb{S}_{+}^{n}$. This just amounts to the introduction of polar coordinates in $x$ and $x^{\prime}$ around $x=x^{\prime}=0$. The space can be covered locally by the two projective coordinate systems with variables $x^{\prime} \geq 0$ and $s \geq 0$, where $s=x / x^{\prime}$ and $x \geq 0$ and $t \geq 0$ where $t=x^{\prime} / x$. In terms of the first of these

$$
A^{\prime}=\frac{1}{(2 \pi)^{n}} \int e^{i \frac{1}{x^{\prime}}\left(\frac{\theta}{s}-\theta^{\prime}\right) \cdot \zeta} a_{L}(\theta / x, \zeta) d \zeta .
$$

Since $\theta, \theta^{\prime} \in \mathbb{S}^{n-1}$ it follows that $\mu=\theta / s-\theta^{\prime} \neq 0$ outside $\left\{\theta=\theta^{\prime}, s=1\right\}$. Integration by parts in $\zeta$ again shows that $A^{\prime}$ is $\mathcal{C}^{\infty}$ (in the new variables $x^{\prime}, s, \theta, \theta^{\prime}$ ) and rapidly decreasing as $x^{\prime} \downarrow 0$. Notice that the amplitude is conormal with respect to $x=0$ so its singularities are swamped by the rapid decrease. As similar analysis holds in the coordinates $x$ and $t$, so $A^{\prime}$ is $\mathcal{C}^{\infty}$ on $X_{\mathrm{b}}^{2}$ away from $\Delta_{\mathrm{b}}$.

The boundary of $\Delta_{\mathrm{b}}$ in $X_{\mathrm{b}}^{2}$, where $X=\mathbb{S}_{+}^{n}$, is precisely $\left\{\theta=\theta^{\prime}, s=1, x^{\prime}=0\right\}$ in these coordinates. Together with $x^{\prime}$ the components of the $n$ vector $\theta / s-\theta^{\prime}=Z$ are local defining functions for $\partial \Delta_{\mathrm{b}}$, so the last blow up (21.2) to define $X_{\mathrm{sc}}^{2}$ is just the introduction of polar coordinates in $\left(x^{\prime}, Z\right)$. The blown up manifold is locally covered by the $n+1$ coordinate systems with coordinates $Z_{j}, x^{\prime} / Z_{j}$ and $Z_{k} / Z_{j}$ for $j=1, \ldots, n$ and $x^{\prime}$ and $u=Z / x^{\prime}$. We concentrate on the last of these, which covers a neighbourhood of the lifted diagonal $\Delta_{\mathrm{sc}}$ represented locally as $u=0$. In terms of these coordinates (22.10) becomes

$$
A^{\prime}=\frac{1}{(2 \pi)^{n}} \int e^{i u \cdot \zeta} a_{L}(\theta / x, \zeta) d \zeta .
$$

This is the Fourier representation of a general conormal distributions with respect to $u=0$ and $x=0$. When the density factors are taken into account this gives 
precisely the same class of kernels as (22.6). The other coordinate patches are easier to analyse.

This discussion is reversible and shows that, in the case of $\mathbb{S}_{+}^{n}$, the two spaces of operators are the same. Since the original definition is by localization, and the definition in (22.6) is obviously coordinate independent the general result follows.

The proof for the 'classical' operators (22.7) is similar, except that the amplitude is smooth down to $x=0$.

The operator wavefront set of a kernel (22.6) is a subset of $N^{*} \Delta_{\mathrm{sc}} \cup \mathrm{ff}\left(X_{\mathrm{sc}}^{2}\right) \equiv C_{\mathrm{sc}} X$ which is empty only for an operator of multiorder $-\infty, \infty$.

\section{Section 23: Composition.}

Although composition properties of the scattering calculus can be shown using the local definition in $\S 4$ we shall briefly discuss the alternate 'global' approach to:

Proposition 18. Each sc-pseudodifferential operator defines a continuous linear operator

$$
\Psi_{\mathrm{scc}}^{m, l}\left(X ;{ }^{\mathrm{sc}} \Omega^{\frac{1}{2}}\right) \ni A: \dot{\mathcal{C}}^{\infty}\left(X ;{ }^{\mathrm{sc}} \Omega^{\frac{1}{2}}\right) \longrightarrow \dot{\mathcal{C}}^{\infty}\left(X ;{ }^{\mathrm{sc}} \Omega^{\frac{1}{2}}\right)
$$

and under composition $\Psi_{\mathrm{scc}}^{*, *}\left(X ;{ }^{\mathrm{sc}} \Omega^{\frac{1}{2}}\right)$ is a bifiltered algebra,

$$
\Psi_{\mathrm{scc}}^{m, l}\left(X ;{ }^{\mathrm{sc}} \Omega^{\frac{1}{2}}\right) \circ \Psi_{\mathrm{scc}}^{m^{\prime}, l^{\prime}}\left(X ;{ }^{\mathrm{sc}} \Omega^{\frac{1}{2}}\right) \subset \Psi_{\mathrm{scc}}^{m+m^{\prime}, l+l^{\prime}}\left(X ;{ }^{\mathrm{sc}} \Omega^{\frac{1}{2}}\right),
$$

with

$$
\Psi_{\mathrm{scc}}^{m, l}\left(X ;{ }^{\mathrm{sc}} \Omega^{\frac{1}{2}}\right)=x^{l} \Psi_{\mathrm{scc}}^{m, 0}\left(X ;{ }^{\mathrm{sc}} \Omega^{\frac{1}{2}}\right) \forall l, m \in \mathbb{R} .
$$

The composition result can be proved by constructing an approriate stretched triple space, the definition of which is similar to that of the double space but more involved. The triple product for the b-calculus is

$$
X_{\mathrm{b}}^{3}=\left[X^{3} ;(\partial X)^{3} ;(\partial X)^{2} \times X ; \partial X \times X \times \partial X ; X \times(\partial X)^{2}\right]
$$

The three partial diagonals, lifted from $X_{\mathrm{b}}^{2}$ by the stretched projections, are psubmanifolds which are each isomorphic to $X_{\mathrm{b}}^{2}$ and which intersect in pairs only in the lift of the triple diagonal. Let $K$ be the boundary of the lifted triple diagonal; it is therefore the intersection of the boundary and any two of the lifted partial diagonals. The three boundary hypersurfaces (assuming for simplicity that $X$ itself only has one boundary hypersurface) of each of the lifted partial diagonals are boundary p-submanifolds of $X_{\mathrm{b}}^{3}$; the part in the front face of the first blow up in (23.4) will be denoted $G_{O}$, for $O=F, S, C$. Of the other two boundary hypersurfaces the one which is in the front face for the corresponding one of the last three blowups in (23.4) will be denoted $J_{O}$. Thus the intersection of any two of the $G_{O}$ is just 
$K$. The $J_{O}$ do not meet each other, but do meet the corresponding $G_{O}$ but do so away from $K$.

With this notation for the lifts of the partial diagonals the triple space can be defined by

$$
X_{\mathrm{sc}}^{3}=\left[X_{\mathrm{b}}^{3} ; K ; \mathcal{G} ; \mathcal{J}\right]=\left[X_{\mathrm{b}}^{3} ; \mathcal{K}\right]
$$

where $\mathcal{G}=\left\{G_{F}, G_{S}, G_{C}\right\}, \mathcal{J}=\left\{J_{F}, J_{S}, J_{C}\right\}$ and $\mathcal{K}=\mathcal{G} \cup \mathcal{J} \cup\{K\}$ has an appropriate order. This iterated blow-up is well defined since on blowing up $K$ the elements of $\mathcal{G}$ become non-intersecting p-submanifolds and the elements of $\mathcal{J}$ lift to non-intersecting p-submanifolds after the first two blow ups. To prove the composition formula for sc-pseudodifferential operators we need the following analogue of Lemma 15:

Lemma 16. There are unique maps, which are b-fibrations, such that the diagram
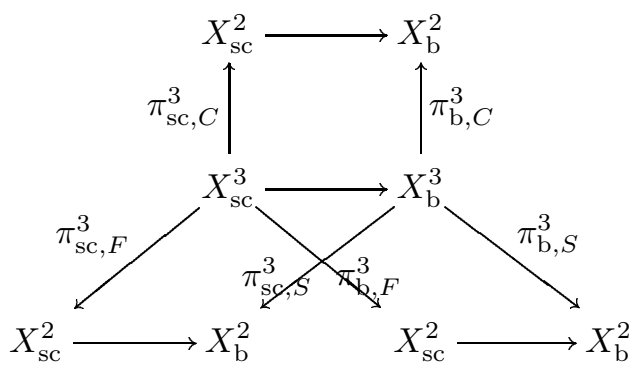

commutes.

Proof: In [8;Chapter 6] it is shown that the b-stretched projections $\pi_{\mathrm{b}, O}^{3}$, for $O=F, S, C$, are b-fibrations such that the diagram underlying (23.6):

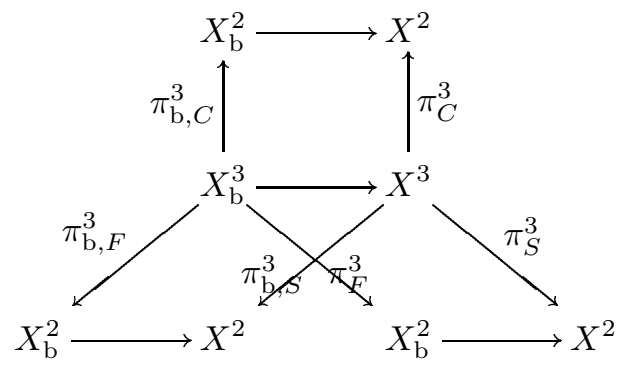

is commutative. Then we can proceed much as in the proof of Lemma 15.

The first task is to define the sc-stretched projections so that (23.6) is commutative. From the inherent symmetry it suffices to define one of the maps, say $\pi_{\mathrm{sc}, F}^{3}$ 
and then show that it is a b-fibration. To do so results on the commutativity of blow up, as in $\S 18$, will also be used.

Writing out all the blow ups in (23.5) gives:

$$
X_{\mathrm{sc}}^{3}=\left[X_{\mathrm{b}}^{3} ; K ; G_{F} ; G_{S} ; G_{C} ; J_{F} ; J_{S} ; J_{C}\right] .
$$

Here the $G$ 's and $J$ 's can be freely commuted, amongst themselves, since they lift to be disjoint in the space in which they are blown up. In fact $J_{F}$ does not meet $G_{S}$ or $G_{C}$ so we also have:

$$
X_{\mathrm{sc}}^{3}=\left[X_{\mathrm{b}}^{3} ; K ; G_{F} ; J_{F} ; G_{S} ; G_{C} ; J_{S} ; J_{C}\right] .
$$

Now $K$ is a submanifold of $G_{F}$ so, using the main commutation result, the order of blow up can be exchanged giving

$$
X_{\mathrm{sc}}^{3}=\left[X_{\mathrm{b}}^{3} ; G_{F} ; K ; J_{F} ; G_{S} ; G_{C} ; J_{S} ; J_{C}\right] .
$$

Recall from (23.4) the definition of $X_{\mathrm{b}}^{3}$, which can be written in the form

$$
X_{\mathrm{b}}^{3}=\left[X^{3} ; I ; B_{F} ; B_{S} ; B_{C}\right]
$$

where $I=(\partial X)^{3}$ and $B_{O}$, for $O=F, S, C$, are the boundary faces $(\partial X)^{2} \times X, X \times$ $(\partial X)^{2}$ and $\partial X \times X \times \partial X$ respectively. The first two blow ups can be interchanged to give

$$
X_{\mathrm{b}}^{3}=\left[X^{3} ; B_{F} ; I ; B_{S} ; B_{C}\right]=\left[X_{\mathrm{b}}^{2} \times X ; I ; B_{S} ; B_{C}\right] .
$$

Inside $X_{\mathrm{b}}^{2} \times X, G_{F}$ meets the lift of $I$ and but does not meet the lift of $B_{S}$ or $B_{C}$. Thus combining (23.12) and (23.10) gives

$$
\begin{aligned}
& X_{\mathrm{sc}}^{3}=\left[X_{\mathrm{b}}^{2} \times X ; G_{F} ; I ; B_{S} ; B_{C} ; K ; J_{F} ; G_{S} ; G_{C} ; J_{S} ; J_{C}\right] \\
& \quad=\left[X_{\mathrm{b}}^{2} \times X ; G_{F} ; I ; J_{F} ; B_{S} ; B_{C} ; K ; G_{S} ; G_{C} ; J_{S} ; J_{C}\right]
\end{aligned}
$$

where the fact that $J_{F}$ does not meet the other $B_{O}$ 's or $K$ has been used. Now in the space $X_{\mathrm{b}}^{2} \times X, I$ is the product of the front face of $X_{\mathrm{b}}^{2}$ and $\partial X, J_{F}$ is the product of the boundary of the lifted diagonal in $X_{\mathrm{b}}^{2}$ and $X$ and $G_{F}$ is the intersection of these two manifolds. Thus the order of blow up can be changed to give

$$
X_{\mathrm{sc}}^{3}=\left[X_{\mathrm{b}}^{2} \times X ; J_{F} ; G_{F} ; I ; B_{S} ; B_{C} ; K ; G_{S} ; G_{C} ; J_{S} ; J_{C}\right] .
$$

Finally then we can use the fact that $J_{F}$ is just the lift from $X_{\mathrm{b}}^{2} \times X$ of the product $\partial \Delta_{\mathrm{b}} \times X$ to write

$$
X_{\mathrm{sc}}^{3}=\left[X_{\mathrm{sc}}^{2} \times X ; G_{F} ; I ; B_{S} ; B_{C} ; K ; G_{S} ; G_{C} ; J_{S} ; J_{C}\right] .
$$


Thus (23.13) allows the sc-stretched projection $\pi_{\mathrm{sc}, F}^{3}$ to be defined as the product of the projection from $X_{\mathrm{sc}}^{2} \times X$ onto $X_{\mathrm{sc}}^{2}$ with the iterated blow-down map from $X_{\mathrm{sc}}^{3}$ to $X_{\mathrm{sc}}^{2} \times X$ given by (23.13). The commutativity of (23.6) follows immediately from this definition.

Proposition 16 can be used show that $\pi_{\mathrm{sc}, F}^{3}$ is a b-fibration. It suffices to show that at each stage of the sequence of blow-ups in (23.13) the composite of the projection to $X_{\mathrm{sc}}^{2}$ with the composite blow-down map at that stage is a b-fibration which is b-transversal to the next submanifold to be blown up with the image of this submanifold meeting the interior of $X_{\mathrm{sc}}^{2}$ or the interior of some boundary hypersurface of $X_{\mathrm{sc}}^{2}$.

Using the sc-stretched projections in (23.6) the composition formula follows from the pull-back and push-forward results of [9] since the composite kernel of $C=A \circ B$ is given by

$$
C=\left(\pi_{\mathrm{sc}, C}^{3}\right)_{*}\left(\left(\pi_{\mathrm{sc}, F}^{3}\right)^{*} A \cdot\left(\pi_{\mathrm{sc}, S}^{3}\right)^{*} B\right) .
$$

\section{Section 24: Symbol map and normal operator.}

The symbol mapping is defined directly from the symbol map for conormal distributions defined by Hörmander in [4], it is also simply the smooth extension of the symbol mapping, in the usual sense, in the interior.

Proposition 19. The symbol mapping for conormal distributions, applied to (22.8) for $l=0$, gives a short exact sequence

$$
0 \longrightarrow \Psi_{\mathrm{sc}}^{m-1,0}\left(X ;{ }^{\mathrm{sc}} \Omega^{\frac{1}{2}}\right) \longrightarrow \Psi_{\mathrm{sc}}^{m, 0}\left(X ;{ }^{\mathrm{sc}} \Omega^{\frac{1}{2}}\right) \stackrel{\sigma_{m}}{\longrightarrow} S^{[m]}\left({ }^{\mathrm{sc}} T^{*} X\right) \longrightarrow 0
$$

which is multiplicative:

$$
\sigma_{m+m^{\prime}}(A \circ B)=\sigma_{m}(A) \cdot \sigma_{m^{\prime}}(B), A \in \Psi_{\mathrm{sc}}^{m, 0}\left(X ;{ }^{\mathrm{sc}} \Omega^{\frac{1}{2}}\right), B \in \Psi_{\mathrm{sc}}^{m^{\prime}, 0}\left(X ;{ }^{\mathrm{sc}} \Omega^{\frac{1}{2}}\right) .
$$

The symbol is principally related to regularity. The normal operator is related to growth near the boundary. On any Euclidian space consider

$$
I^{m} \mathcal{S}\left(\mathbb{R}^{n}\right)=I_{c}^{m-\frac{1}{4} n}\left(\mathbb{R}^{n} ;\{0\}\right)+\mathcal{S}\left(\mathbb{R}^{n}\right)
$$

where the first term is the space of conormal distributions of compact support associated to $\{0\}$. This is obviously invariant under linear transformations so can be extended to a similar space on the fibres of a vector bundle $U$ over a manifold with boundary $X$ by setting

$$
I^{m} \mathcal{S}(U)=I_{c}^{m+\frac{1}{4}(N-n)}(U ; O)+\mathcal{S}(U)
$$

where $N$ and $n$ are the base and fibre dimensions of $U, O$ is the zero section of $U$ and $\mathcal{S}(U) \subset \mathcal{C}^{\infty}(U)$ is the space of smooth functions which decrease rapidly at infinity, with all derivatives. This is always a filtered algebra, with the product given by convolution on the fibres. 
Proposition 20. There is a well-defined normal operator, $N_{\mathrm{sc}}$, on the small calculus which gives a short exact sequence for each $m, l$

$$
0 \longrightarrow \Psi_{\mathrm{sc}}^{m, l+1}\left(X ;{ }^{\mathrm{sc}} \Omega^{\frac{1}{2}}\right) \longrightarrow \Psi_{\mathrm{sc}}^{m, l}\left(X ;{ }^{\mathrm{sc}} \Omega^{\frac{1}{2}}\right) \stackrel{N_{\mathrm{sc}}}{\longrightarrow} I^{m} \mathcal{S}\left({ }^{\mathrm{sc}} T_{\partial X} X ;\left|N^{*} \partial X\right|^{l}\right) \longrightarrow 0
$$

and which is a filtered algebra homomorphism.

From the fact that both the symbol map and normal operator are defined by commutative homomorphism the commutator of two operators is of lower order in both senses:

$$
\begin{gathered}
A \in \Psi_{\mathrm{sc}}^{m, k}\left(X ;{ }^{\mathrm{sc}} \Omega^{\frac{1}{2}}\right) \text { and } B \in \Psi_{\mathrm{sc}}^{m^{\prime}, l}\left(X ;{ }^{\mathrm{sc}} \Omega^{\frac{1}{2}}\right) \\
\Longrightarrow[A, B] \in \Psi_{\mathrm{sc}}^{m+m^{\prime}-1, k+l+1}\left(X ;{ }^{\mathrm{sc}} \Omega^{\frac{1}{2}}\right)
\end{gathered}
$$

For application to the limiting absorption principle it is important to find a formula for the normal operator of such a commutator. The symbol of the commutator is given directly in terms of the Poisson bracket of the symbols and a similar result holds for the normal operator.

Proposition 21. For the commutator in (24.6)

$$
\widehat{N}_{\mathrm{sc}}([A, B])=\frac{1}{i}\left\{\widehat{N}_{\mathrm{sc}}(A), \widehat{N}_{\mathrm{sc}}(B)\right\}_{\mathrm{sc}}
$$

where the Poisson bracket extends by continuity from the interior to define a bilinear form

$$
\left.x^{k} \mathcal{C}^{\infty}\left({ }^{\mathrm{sc}} T^{*} X\right) \times x^{l} \mathcal{C}^{\infty}{ }^{(\mathrm{sc}} T^{*} X\right) \longrightarrow x^{k+l+1} \mathcal{C}^{\infty}\left({ }^{\mathrm{sc}} T^{*} X\right)
$$

and hence restricts to the boundary to give

$$
\mathcal{C}^{\infty}\left({ }^{\mathrm{sc}} T_{\partial X}^{*} X ;\left|N^{*} \partial X\right|^{k}\right) \times \mathcal{C}^{\infty}\left({ }^{\mathrm{sc}} T_{\partial X}^{*} X ;\left|N^{*} \partial X\right|^{l}\right) \longrightarrow \mathcal{C}^{\infty}\left({ }^{\mathrm{sc}} T_{\partial X}^{*} X ;\left|N^{*} \partial X\right|^{k+l}\right) .
$$

\section{BIBLIOGRAPHY}

1. J.J. Kohn and L. Nirenberg, On the algebra of pseudo-differential operators, Comm. Pure Appl. Math. 18 (1965), 269-305.

2. L. Hörmander, Pseudo-differential operators, Comm. Pure Appl. Math. 18 (1965), 501-517.

3. L. Hörmander, On the existence and the regularity of solutions of linear pseudo-differential equations, Ens. Math. 17 (1971), 99-163.

4. L. Hörmander, Fourier integral operators, I, Acta Math. 127 (1971), 79-183.

5. L. Hörmander, The Weyl calculus of pseudo-differential operators, Comm. Pure Appl. Math. 32 (1979), 359-443.

6. L. Hörmander, The analysis of linear partial differential operators III, Springer Verlag, 1985.

7. R.B. Melrose, The Atiyah-Patodi-Singer index theorem, A. K. Peters, Boston, 1993.

8. Differential analysis on manifolds with corners, In preparation.

9. Calculus of conormal distributions on manifolds with corners, International Mathematics Research Notices (1992 No.3), 51-61. 
10. R.B. Melrose and G.A. Mendoza, Elliptic operators of totally characteristic type, MSRI Preprint (1983).

11. E. Mourre, Absebce of singular spectrum for certain self-adjoing operators, Comm. Math. Phys 78 (1981), 391-400.

12. D. Robert, Relative time-delay fore perturbations of elliptic operators and semiclassical asymptotics, Preprint.

13. D. Yafaev, Resolvent estimates and scattering matrix for $N$-particle hamiltonians, Preprint 1993. 